NBER WORKING PAPER SERIES

\title{
LISTING ADVANTAGES AROUND THE WORLD
}

\author{
Kenichi Ueda \\ Somnath Sharma \\ Working Paper 26446 \\ http://www.nber.org/papers/w26446 \\ NATIONAL BUREAU OF ECONOMIC RESEARCH \\ 1050 Massachusetts Avenue \\ Cambridge, MA 02138 \\ November 2019
}

The views expressed in this paper are those of the authors and should not be attributed to any institutions that the authors have been affiliated with, nor of the National Bureau of Economic Research. This work is supported by the GraSPP Research Grant EGRM-20160 and the CARF at the University of Tokyo. This paper is also conducted as a part of the project "Study on Corporate Finance and Firm Dynamics" undertaken at the Research Institute of Economy, Trade and Industry (RIETI). We are grateful for helpful comments from Yishay Yafeh and participants of the NBER-TCER-CEPR (TRIO) Conference at the University of Tokyo.

NBER working papers are circulated for discussion and comment purposes. They have not been peer-reviewed or been subject to the review by the NBER Board of Directors that accompanies official NBER publications.

(C) 2019 by Kenichi Ueda and Somnath Sharma. All rights reserved. Short sections of text, not to exceed two paragraphs, may be quoted without explicit permission provided that full credit, including $(\subset$ notice, is given to the source. 
Listing Advantages Around the World

Kenichi Ueda and Somnath Sharma

NBER Working Paper No. 26446

November 2019

JEL No. E22,G32

\title{
ABSTRACT
}

Using the firm-level data of 33 countries over 10 years (from 2008-2017), we find that the listed firms, on average, have lower marginal products of capital (measured by return on assets) than the unlisted firms in many countries. This implies that the listed firms face less financial constraints. Moreover, we investigate the institutional factors that exacerbate or mitigate the listing advantages across the countries. The listing advantages seem enlarged with better corporate governance and narrowed with stronger creditor's rights.

\author{
Kenichi Ueda \\ The University of Tokyo \\ 7-3-1 Hongo Bunkyo-ku \\ Tokyo 113-0033 \\ Japan \\ ueda.econ@gmail.com
}

Somnath Sharma

University of Tokyo 


\section{Introduction}

Using the firm-level data of 33 countries of 10 years (from 2008-2017) for both listed and unlisted firms, we find that the listed firms, on average, have lower marginal products of capital than the unlisted firms in many countries. We call it listing advantages.

For each country, we estimate the listing advantages by the average treatment effect on the return on asset $(R O A)$ using the propensity score matching technique. We find that, for the majority of the countries in our study, listing lowers $R O A$, which is proportional to the marginal products of capital under typical assumptions on production functions. In other words, the marginal product of capital of the listed firms are lower than those of the unlisted firms that have similar characteristics.

This finding supports a simple theory, which says that the listing firms should be less financially constrained. As long as diminishing marginal returns prevail, any financial constraints limiting investments raise the marginal product of capital of a firm more than its unconstrained level. Indeed, in many countries, listing is negatively correlated with debt-toasset ratio, implying that equity finance works as a source of financing. And, firms maintain lower leverage, which makes listed firms less likely to hit the prohibitive borrowing constraints. $^{1}$

Next, we investigate the institutional factors behind different levels of listing advantages across countries. We find that better corporate governance tends to improve the listing advantages, while stronger creditor's rights, and perhaps easiness of starting up business, seem to narrow them.

Although cross-country studies are rare, several country-specific studies, so far, identify

\footnotetext{
${ }^{1}$ Ueda et al. (2019) more robustly find these results in a detailed study focusing on Japanese firms.
} 
the advantages of listing. Ueda et al. (2019), using Japanese firm-level data over the period 1995-2014, find that listing can mitigate the financial constraints of firms. Compared to a similar unlisted firm, a listed firm has a lower marginal product of capital, especially in recessions. Schoubben and Van Hulle (2011), using the Belgian firm-level data (from 1992 to 2005) with consolidated financial statements, find that listing provides more flexibility in financing and reduces the financial constraints. Gilje and Taillard (2016), based on panel regressions, show that listed natural gas firms in the U.S. have a higher sensitivity to gas price movements (i.e., growth opportunities) than the unlisted rivals in the same industry. The difference is more pronounced in shale gas investments, which are more capital intensive. For British firms, Saunders and Steffen (2011) find that the listed firms enjoy lower bank loan rates. In their European cross-country study, Mortal and Reisel (2013) report that the listed firms have higher investment sensitivity on growth opportunities (proxied by sales growth) and such tendencies are higher for countries with more developed stock markets.

Other authors find that listing results in tightening of financial constraints. Stein (1989), argue that it may be possible theoretically as a result of the worsening of agency problems under sparse ownership. For the U.S. firms, Asker et al. (2014), show that U.S.-listed firms are more short-termist in nature, that is, they are less sensitive to growth opportunities (proxied by sales growth). Sheen (2016), also shows that the U.S. listing firms in chemical industries have a lower sensitivity of capacity investment on demand shocks.

Mixed evidences are sometimes reported by several authors. For British firms, Brav (2009), shows that listed firms have lower leverage, but with lower fluctuations in the capital structure. In their European cross country study, Goyal et al. (2011) also report that listed firms have lower leverage, but with more active management on leverage. They find that this difference between the listed and the unlisted firms are more pronounced in countries with stronger creditor's rights. For Japanese firms, Orihara (2014) presents univariate pictures 
that show the listed firms have lower investments on average but with lower fluctuations in investment over business cycles. Orihara and Isobe (2014), report that the listed firms have lower leverage, though with minimal control variables in their regressions.

Based on listed firms' data, many authors have explained the cross-country differences in financial frictions based on the differences in institutional factors. Claessens et al. (2014), using an improved version of the standard investment model, estimate how institutions affect financial frictions at the firm (micro) level and, through the required rate of return, at the country (macro) level. Based on listed firm-level panel data from 40 countries over the period 1990 to 2007, they show that shareholder rights affect financial frictions while creditor's rights do not. Abiad et al. (2008), show that the cross-sectional dispersion of listed-firms' Tobin's Q, which is regarded as a measure of the ex-ante efficiency of capital allocation, improves with financial liberalizations.

Few, if not none, so far, have studied the role of institutional factors on financial constraints between the listed and the unlisted firms using the cross-country panel data. In this paper, using both listed and unlisted firms data, we thus try to find extensive margins of institutional factors on listing, compared to intensive margins found so far in previous studies based on listed-firm panel data in cross-country setting.

\section{Data}

\subsection{Firm-level Data}

We use two levels of data-country-wise firm-level and country-level data. For the countrywise firm-level data, we use the ORBIS database which is provided commercially by Bureau van Dijk (BvD). The ORBIS database includes balance sheets and income statements together with information on firms' ownership structures for over 300 million companies across 
the world. In terms of downloading and cleaning the data from ORBIS, we follow KalemliOzcan et al. (2015) as much as possible to prepare nationally representative data with minimal missing information. For our purpose, we use the data from 33 countries for manufacturing firms from 2008-2017. We restrict our attention to the firms with at least 2 years of consecutively available data points. In other words, this data set is an unbalanced panel. We also eliminate the pure subsidiaries, i.e., if a firm's immediate shareholder owns more than 50 percent of the shares.

Following the literature (Hayashi, 1982), we take it as granted that the marginal product of capital (MPK) is proxied by the average product of capital, assuming typical production functions exhibiting constant returns to scale in factor inputs. We use the return on assets $(R O A, \%)$ as a benchmark proxy for the average and marginal products of capital because a firm should utilize all the assets efficiently. We estimate the $R O A$ by earnings before interest and tax payments (EBIT) divided by Total Assets(TA), i.e. $R O A=E B I T / T A .^{2}$

In reality, to ease the financing constraints, especially for possible liquidity needs in case of distress, firms may save and hold some amounts of cash and equivalents, which yield very low returns in general. If so, it makes sense to exclude liquid assets from the Total Assets(TA) and to focus on the return on fixed assets $(R O K, \%)$ as another proxy for the marginal products of capital, that is, $R O K=E B I T /$ Fixed Assets.

We use dummy Listing $=1$ if the firm is listed on the stock market and Listing $=0$ otherwise. This is the listing variable. We use Total Assets a measure of the Size of a firm and the total number of years from the year of incorporation as the Age of the firm. For Leverage, we use the debt-to-asset ratio $(D / A$ Ratio).

\footnotetext{
${ }^{2}$ The $R O A$ given in the ORBIS database is profit and loss before tax but after the interest payments.
} 
We drop outliers based on $R O A$ and $R O K$. That is, samples showing smaller (or larger) values than three standard deviations from the averages in terms of the return on asset $(R O A)$ and the return on fixed assets $(R O K)$. We present the number of firm-year observations by country in Table 1a.

\subsection{Country-level Data}

At the country-level, for each year, we take several institutional indicators for corporate governance, institutional quality, product market competition, and financial development from various sources. They are explained below and summarized in Table 1b. We present the correlation table for the cross-country analysis in two parts in Table 1c (part a and b). Notably, Table 1c shows that the average difference of marginal products of capital between the listed and the unlisted firms is negatively associated with the majority of the institutional indicators. We report the country-wise summary statistics and correlation tables of these variables in Appendix A1.

\subsubsection{Corporate Governance}

For corporate governance, we use six different indicators, namely, Self-dealing Index, Corporate Governance Quality Index, Anti-Directors Rights Index, Extent of Directors Liability, Corporate Board Efficacy Index, and Protection of Minority Shareholders Index.

- Self-dealing Index: Self-dealing index provided by Djankov et al. (2008). It is a measure of legal protection of minority shareholders against expropriation by corporate insiders (for example, owner's transactions with related parties). This index predicts a variety of stock market outcomes, and is generally known to work better than other measures of corporate governance e.g. anti-director rights.

- Corporate Governance Quality Index: We take this data from De Nicolò et al. (2008). It is a composite indicator related to accounting transparencies. It measures 
strength of auditing and accounting standards conflict of interest regulation and shareholder governance.

- Anti-director Rights Index: We take this indicator of minority shareholder's protection from Spamann (2009) which is a revised and improved version of La Porta et al. (1998). It has six components. Three are concerned with shareholder voting - voting by mail, voting without blocking of shares, and calling an extraordinary meeting, and, three with shareholders' voices - proportional board representation, preemptive rights, and judicial remedies.

- Protection of Minority Shareholders: This indicator is a measure of whether minority shareholders are protected by the legal system of the country. This is constructed on the basis of opinion surveys of business leaders around the world. We take this indicator from World Economic Forum.

- Corporate Board Efficacy: This indicator measures how well we can characterize corporate board by investors and boards of directors. We take this indicator from World Economic Forum.

- Extent of Director's Liability: The extent of director's liability index measures when board members can be held liable for harm caused by related-party transactions and what sanctions are available. We take this indicator from World Bank's Doing Business Reports.

\subsubsection{Creditor's Rights}

We use four different indicators of Creditor's Rights. They are, Creditor's Rights, Strength of Legal Rights, Strength of Insolvency Framework, and Time to Resolve Insolvency (years).

- Creditor's rights: We use the creditor's rights as given in the Djankov et al. (2008). The creditor rights index first proposed by La Porta et al. (1998). The index measures 
the legal rights of creditors against defaulting debtors in different jurisdictions and has been previously interpreted as a measure of creditor power.

- Strength of Legal Rights: We take this indicator from World Bank's Doing Business Reports. This index measures the degree to which collateral and bankruptcy laws protect the rights of borrowers and lenders and thus facilitate lending. This measure weigh borrower side compared to Creditor's Rights.

- Strength of Insolvency Framework: Strength of insolvency framework index measures the time, cost, and outcome of insolvency proceedings involving domestic entities as well as the strength of the legal framework applicable to judicial liquidation and reorganization proceedings. We take this indicator from World Bank's Doing Business Reports.

- Time to Resolve Insolvency (years): It is the number of years from the filing for insolvency in court until the resolution of distressed assets. We take this indicator from World Bank's Doing Business Reports. This one and the one above measures overall efficiency of bankruptcy procedures.

\subsubsection{General Institutional Quality}

We use three different indicators of General Institutional Quality. They are-Property Rights, Rule of Law, and Trust in People.

- Property Rights: We use the property rights index data published by the World Economic Forum. This data is part of Global Competitiveness Report and is collected through survey of different stakeholders across the world. It takes into account the protection of property rights including financial assets.

- Rule of Law: This indicator captures the perceptions of the extent to which agents have confidence in and abide by the rules of society, and in particular the quality 
of contract enforcement, property rights, the police, and the courts, as well as the likelihood of crime and violence. We take this from the World Governance Indicators published by the World Bank.

- Trust in People: We take this data from two different waves of the World Value Surveys (Wave 5 (2005-2009) and Wave 6 (2010-2014)). The World Value Survey publish time series investigation of human beliefs and values across countries. We take the indicator constructed by response of the respondents on - whether most people can be trusted in your country.

\subsubsection{Financial Development}

We take four different indicators of financial development. They are, Credit-to-GDP Ratio, Sum of stock market capitalization, private bond market capitalization, and bank credit to Private Sector (\% of GDP), Market Capitalization (\% of GDP), and Prevalence of Foreign Ownership.

- Credit-to-GDP Ratio: We take private sector Credit-to-GDP ratio (\%) from the World Bank database. Credit here refers to the domestic credit to private sector which includes financial resources provided to the private sector by financial corporations through loans, purchases of non-equity securities, and trade credits and other accounts receivable, which establish a claim for repayment.

\section{- Sum of stock market capitalization, private bond market capitalization and} bank credit to private sector (\% of GDP): We take bank credit to private nonfinancial sector data and outstanding corporate debt securities issued by the private non-financial corporations data given by the Bank for International Settlements (BIS) and market capitalization of the listed companies data from the World Bank and CEIC Database and take the sum of them and divide it by current GDP at market price of that year to obtain this ratio. 
- Market Capitalization (\% of GDP): We take this data from the World Bank and the CEIC Database. Market capitalization (also known as market value) is the share price times the number of shares outstanding (including their several classes) for listed domestic companies.

- Prevalence of Foreign Ownership: We use Prevalence of Foreign Ownership index provided by the World Economic Forum. It is constructed on the basis of responses from the survey of the stakeholders in each country on how prevalent is foreign ownership of companies in their respective countries.

\subsubsection{Product Market Competition}

We take three different indicators of product market competition. They are, New Business Registered (new registrations per 1,000 people ages 15-64), Cost of starting a Business(\% of income per capita), and Trade Barriers.

- New Business Registered: We take new business registrations in the calendar year per 1000 people in the age group of 15-64 years published by the World Bank. Business refers to limited liability corporations. This measure captures easiness of starting up business, together with the measure below.

- Cost of Starting a Business (\% of income per capita): We take this data from the World Bank. This is constructed by normalizing the cost to register a business by the gross national income (GNI) per capita.

- Trade Barriers: This index is constructed based on the responses of the stakeholders in each country to the question - to what extent do non-tariff barriers (e.g., health and product standards, technical and labeling requirements, etc.) limit the ability of imported goods to compete in the domestic market? We take this data from the World Economic Forum. 


\subsubsection{Macro Volatility}

We take three measures of macroeconomic volatility - Standard Deviation of Inflation from Last 5 Years Average Inflation, Standard Deviation of GDP growth from Last 5 Year Average GDP Growth, and Coefficient of variation of Exchange Rate. We use the annual average consumer price index to calculate inflation. For calculating the GDP growth, we use GDP at constant market price (in USD million). We take nominal effective exchange rate to calculate the coefficient of variation of the exchange rate. We use the World Bank database to obtain these variables.

\section{Within-Country Estimation of Average Treatment Effect on Treated: Propensity Score Matching}

\subsection{Benchmark Within-Country Estimation}

Following Ueda et al. (2019) study on Japanese firms, we conduct a propensity score matching estimation (PSM) to identify the listing advantages for each year for each of the 33 countries. Specifically, we first predict the probability of firms to be listed based on Size (total asset), Age, Industry (2-digit level) and Leverage (D/A Ratio). Second, we match the listed and the unlisted firms, one to one, if they share the (almost) same probability of being listed. ${ }^{3}$ We confirm that covariates are well balanced in matched samples (report omitted). Third, we compare the difference in the variables of interest i.e $R O A$ between two matched samples to determine the effects of listing.

The marginal product of capital, is proxied by the return on asset $(R O A)$. The key variable is the binary variable, Listing, taking value one if listed and zero otherwise. For the matching conditions, we use Size and Age. We also include lagged Leverage to control

\footnotetext{
${ }^{3}$ We use STATA 14 command psmatch2 with caliper 0.1 .
} 
for possible default risks, debt overhang, and ROE targeting behaviors.

In Table 2, we present the average treatment effect on treated (ATT) which is the average difference of in the $R O A$ as a result of being listed, for each year and each country. In Figure 1, we report the year-average ATT for each country based on at least $10 \%$ level of significance.

We find that, on average, the difference of the $R O A$ as a result of Listing is mostly negative for almost all the countries. In other words, on an average, the listed firms tend to have a lower $R O A$ compared to the unlisted firms. This difference is more pronounced in the case of Australia, Ireland, Romania, Sweden, the U.S. and the U.K.. For some countries like Brazil, Finland, Hungary, Greece, India, Malaysia, Norway and Taiwan, this difference is not found to be statistically significant. There are few exceptions (namely, Vietnam and Iceland), where this difference turns out to be positive. ${ }^{4}$

\subsection{Robustness Checks for Within-Country Estimation}

We notice a caveat for our propensity score matching estimates. On the one hand, Age and Industry can be regarded as pre-determined or almost exogenous to the firm's decision on Listing. On the other hand, Size (total asset) may be endogenous to Listing. However, it is a slow-moving variable which is less likely to affect the Listing decision in each year. We restrict our sample to firms that are more than 5 years of age to eliminate young firms, which are more likely to face the listing decision. We do not find any difference in our results.

Still, we care about possible endogeneity of listing. We note that the treated and the controlled are switched, in a sense, at 50 percent probability of being listed. For firms higher than 50 percent score, they should be listed according to the statistical model. The differ-

\footnotetext{
${ }^{4}$ Similar results are obtained when we pool all years observations for each country.
} 
ence between the listed and the unlisted can be interpreted as the opportunity loss for the unlisted firms not being listed, though they should be. On the other hand, for firms lower than 50 percent score, they should not be listed according to the statistical model. They may pick up distressed firms, which may exaggerate the benefits of listing. Also, these listed firms may be close to being delisted, so, even though listed, they have difficulties in obtaining funds. Hence, the bias is uncertain. In other words, randomness of listing seems to appear more clearly among those samples above the 50 percent threshold.

Note that the underlying assumption for justifying propensity score matching estimation is that the listing status is not based on the economic reasons, at least not on the current economic factors. And, assignment of such non-economic reasons across firms are assumed to be random, at least on the basis of observed characteristics (Age, Size, Industry, and Leverage). For example, some owner-managers of the unlisted firms may enjoy non-economic benefits and adamantly refuse to be listed. The assignment of these non-economic benefits to owner-managers of firms can be considered as random. These firms appear more when we take firms with 50 percent or more propensity score of Listing.

We investigate whether the beneficial effects based on all firms are similarly found in firms with more than 50 percent propensity score. We find that correlation coefficient between the overall ATT and the ATT estimated based on more than 50 percent propensity score is around 0.861. These estimates are reported in Appendix Tables A2.1-2.3. Among the firms with higher than 50 percent propensity score, the listing effects are broadly consistent with our benchmark results. This is also in line with Ueda et al. (2019) on Japanese data. Therefore, theoretically, although the random assignment assumption of listing status might not be perfectly solid, we can regard the benchmark results based on all firms as if it is having a random assignment. 
Depending on the macroeconomic conditions, listing advantages may change. Indeed, Ueda et al. (2019) report that the listing advantage is more pronounced in recessions. To purge the recession-boom effects, it would be better to look at the annual estimates. Also, we surmise that the institutional changes over time may matter. Below, we use cross-country panel regressions to allow for these time-varying possibilities.

\subsection{Robustness Check Using Fixed-Effect Panel Estimation}

As another robustness check, we also run the fixed-effect panel estimation for listing advantages for each country. Note that the distribution of the listed firms is skewed towards larger ones while that of the unlisted is towards smaller ones. ${ }^{5}$ This asymmetric distribution potentially causes bias to fixed-effect-regression estimates because the error terms of the listed and the unlisted might not be randomly distributed even with Listing binary variable and other control variables in the regressions:

$$
M P K_{i, j, t}=\alpha_{j}^{M}+\beta^{M} \text { Listing }_{i, j, t}+\gamma_{1}^{M} \text { Size }_{i, j, t-1}+\gamma_{2}^{M} \text { Age }_{i, j, t}+\gamma_{3}^{M} \text { Leverage }_{i, j, t-1}+\epsilon_{i, j, t}^{M},
$$

where $i$ denotes each firm, $j$ industry, and $t$ year.

In Figure 2, we present the estimates (statistically significant) of $\beta^{M}$ (the marginal effect of Listing on the $M P K) .{ }^{6}$ The estimation results show that the listed firms face less financial frictions as in PSM estimates of ATT in many countries. We also find that the average difference of $M P K$ due to the listing based on ATT of PSM and coefficient of listing in the fixed effect regression (i.e. $\beta^{M}$ in equation (1)) are positively correlated, with correlation coefficient 0.614 (Table A2.4, Appendix A2).

\footnotetext{
${ }^{5}$ See Appendix 1 where country-wise correlation tables are reported.

${ }^{6}$ Appendix A3 reports the country-wise results for manufacturing firms. The robust standard errors are reported with clustering at the 2-digit industry level. The coefficients on the listing are significantly negative for almost all the countries when we control for Size, Age and Leverage.
} 


\section{Cross-country Panel Regressions to Investigate the Underlying Factors}

\subsection{Benchmark Cross-country Regression}

To investigate the factors affecting listing advantages, we regress the annual ATT estimates on corporate governance, creditor's rights, institutional quality, product market competition, financial market development, macroeconomic volatility, inflation and interest rates.

$$
\begin{aligned}
& A T T_{k, t}=\alpha_{k}+\beta_{1} \text { CorporateGovernance }_{k, t}+\beta_{2} \text { Creditor }^{\prime} \text { sRights }_{k, t} \\
& +\beta_{3} \text { InstitutionalQuality }_{k, t}+\beta_{4} \text { Product MarketCompetition }_{k, t} \\
& +\beta_{5} \text { FinancialMarketDevelopment }_{k, t}+\beta_{6} \text { MacroVolatility }_{k, t} \\
& +\beta_{7} \text { In }_{\text {flation }_{k, t}}+\beta_{8} \text { InterestRate }_{k, t}+\nu_{k, t},
\end{aligned}
$$

where $k$ denotes country and $t$ year, and $\alpha_{k}$ represents the country fixed effect. We report robust standard errors with clustering at the country-level. Note that, for the cross-country study, we did not use ATT for countries in which more than 90 percent of the sample firms are listed, namely, India, Taiwan, and the U.S..

We present the benchmark regression results in the column 1 of Tables 3.a to 3.f for easy comparison with the other formulations. For the benchmark regression (2), we use the following variables: Self-dealing Index for Corporate Governance; Creditor's Rights for Creditor's Rights; Property Rights for Institutional Development; Credit-to-GDP Ratio for Financial Development; Number of New Business Registered for Product Market Competition; and Standard Deviation from last 5 years Average Inflation for Macroeconomic Volatility. ${ }^{7}$

We find that the countries with better self-dealing index have larger difference in $R O A$

\footnotetext{
${ }^{7}$ Not all the institutional variables are time varying, specifically, Anti-director's rights index, Corporate Governance Quality Index, Self-dealing Index, and Creditor's Rights Index.
} 
due to listing. This implies that in the countries where the legal protection of minority shareholders against expropriation by corporate insiders (for example, owner-manager's related party transactions) is better, the advantage due listing is more. In the countries where the creditor's rights are stronger, the difference in $R O A$ due to listing is smaller. The number of new business registered, which measures easiness to start up business, also narrows the listing advantages. This implies easier the of starting up of business, narrower would be the listing advantages. Other variables, that is, general institutional quality, financial market development and macroeconomic volatility are found to be statistically insignificant.

\subsection{Robustness Checks: Cross-country Regression}

To verify that our results are not driven by the specific firm and country measures as well as by the characteristics of the sample, we examine a number of alternative specifications. For this, we change the indicators of each of the variables one-by-one and compare the results with the benchmark.

In the benchmark, we use the ATT estimates using the propensity score match based on all firms in each country. However, as we discussed above, listed firms with the probability of listing less than 50 percent are likely financially distressed, and low $R O A$ for those firms might not be regarded as random assignment. So, we run a regression focusing only on firms with a probability of listing more than 50 percent. We obtained almost the same result (report omitted).

Next we use five more indicators for Corporate Governance, they are, Corporate Governance Quality Index, Anti-Director's Rights Index, Extent of Director's Liability, Corporate Board Efficacy Index, and Protection of Minority Shareholders Index, in addition to Selfdealing Index. Results are shown in Table 3a, columns 2-6. Overall, estimates does not seem robust - some are positive others are negative and the rest are statistically insignifi- 
cant. However, among these indicators, (anti-)self dealing index and corporate governance quality index measure more directly the restrictions on owner-manager behaviors. For these direct measures, corporate governance do matter.

For creditor's rights, we use four different indicators - Creditor's Rights, Strength of Legal Rights, Strength of Insolvency Framework, and Time to Resolve Insolvency (years). The results are shown in Table 3b. Recall that the Strength of Legal Rights reflect not only creditor's rights but also borrower's rights, while Strength of Insolvency Framework and Time to Resolve Bankruptcy capture the overall efficiency of the bankruptcy procedure. The result shows that more borrower's rights seems to enlarge the listing advantages. The overall efficiency of bankruptcy procedure seem to narrow the listing advantages. These results are consistent with the benchmark results, that is, the Creditor's Rights have narrowing effect on the listing advantage.

For the product market competition, we use two other indicators that reflect the ease of market entry, namely, the Cost of Starting Business and Trade Barriers but we do not find these variables to significantly affect the listing advantages (Table 3.e).

Other variables are statistically insignificant. For institutional quality, we use the Rule of Law instead of Property Rights and find essentially the same insignificant result (Table 3c, column 10). For the financial market development, we use three different indicatorsthe Sum of Stock Market Capitalization, Private Bond Market Capitalization and Bank Credit over GDP; The Stock Market Capitalization (over GDP); and Prevalence of Foreign Ownership. Results are given in Table 3d. For the macroeconomic conditions, macro volatilities, the level of the real interest rate and inflation does not seem to matter (Table 3f). 


\section{Conclusion}

Based on propensity score matching estimations for annual data for each country, we find that listed firms enjoy greater access to finance, which lowers marginal products of capital on average in 23 countries out of 33 sample countries. Other countries show little listing advantages, except that listed firms in Vietnam (and perhaps, Iceland) seem to have less access to finance. The effect is essentially the same for those less endogenous samples, that is, the set of firms with 50 percent or more propensity of being listed. The fixed-effect regressions broadly replicate these findings too. We surmise that, in the majority of countries, the private firm owners adamantly resisting listing would have some benefits to do so, but at the same time, we confirm that they face tighter financial constraints.

We gauge the listing advantages as the average treatment effect on treated (ATT) on $R O A$ for each sample country. We then investigate factors behind the listing advantages based on cross-country panel regressions. Better corporate governance, especially those which directly restrict the owner-manager behaviors, increase the listing advantages. Stronger creditor's rights (and weaker borrower's rights) lower the listing advantages. Easiness of starting up new business has a similar effect. However, other factors like macroeconomic instability and general institutional quality have no significant effects on the listing advantages.

These effects on the extensive margin is partially in line with the intensive margin of credit allocation efficiency among listed firms reported by Claessens et al. (2014), which shows that the primary factors affecting financial frictions for listed firms is country-specific corporate governance as well as the positive impact of product market competition. However, Claessens et al. (2014) find no effect of creditor's rights.

In essence, the listing advantages are the extensive margin of the financial constraints. They are the costs for a firm remaining private. Our results show that such a wedge exists 
almost everywhere in the world. Moreover, the wedge between the listed and the unlisted seems more extensive in a country with better corporate governance, whereas they are narrower in a country with stronger creditor's rights. 


\section{References}

Abiad, A., Oomes, N., and Ueda, K. (2008). The quality effect: Does financial liberalization improve the allocation of capital? Journal of Development Economics, 87(2):270-282.

Asker, J., Farre-Mensa, J., and Ljungqvist, A. (2014). Corporate investment and stock market listing: A puzzle? The Review of Financial Studies, 28(2):342-390.

Brav, O. (2009). Access to capital, capital structure, and the funding of the firm. The Journal of Finance, 64(1):263-308.

Claessens, S., Ueda, K., and Yafeh, Y. (2014). Institutions and financial frictions: Estimating with structural restrictions on firm value and investment. Journal of Development Economics, 110:107-122.

De Nicolò, G., Laeven, L., and Ueda, K. (2008). Corporate governance quality: Trends and real effects. Journal of Financial Intermediation, 17(2):198-228.

Djankov, S., La Porta, R., Lopez-de Silanes, F., and Shleifer, A. (2008). The law and economics of self-dealing. Journal of financial economics, 88(3):430-465.

Gilje, E. P. and Taillard, J. P. (2016). Do private firms invest differently than public firms? taking cues from the natural gas industry. The Journal of Finance, 71(4):1733-1778.

Goyal, V. K., Nova, A., and Zanetti, L. (2011). Capital market access and financing of private firms. International Review of Finance, 11(2):155-179.

Hayashi, F. (1982). Tobin's marginal q and average q: A neoclassical interpretation. Econometrica: Journal of the Econometric Society, pages 213-224.

Kalemli-Ozcan, S., Sorensen, B., Villegas-Sanchez, C., Volosovych, V., and Yesiltas, S. (2015). How to construct nationally representative firm level data from the orbis global database. Technical report, National Bureau of Economic Research.

La Porta, R., Lopez-de Silanes, F., Shleifer, A., and Vishny, R. W. (1998). Law and finance. Journal of political economy, 106(6):1113-1155.

Mortal, S. and Reisel, N. (2013). Capital allocation by public and private firms. Journal of Financial and Quantitative Analysis, 48(1):77-103.

Saunders, A. and Steffen, S. (2011). The costs of being private: Evidence from the loan market. The Review of Financial Studies, 24(12):4091-4122.

Schoubben, F. and Van Hulle, C. (2011). Stock listing and financial flexibility. Journal of Business Research, 64(5):483-489.

Sheen, A. (2016). Do public and private firms behave differently? an examination of investment in the chemical industry. An Examination of Investment in the Chemical Industry (March 23, 2016).

Spamann, H. (2009). The antidirector rights index revisited. The Review of Financial Studies, 23(2):467-486.

Stein, J. C. (1989). Efficient capital markets, inefficient firms: A model of myopic corporate behavior. The Quarterly Journal of Economics, 104(4):655-669.

Ueda, K., Ishide, A., and Goto, Y. (2019). Listing and financial constraints. Japan and the World Economy, 49:1-16. 


\section{Figures and Tables}

Figure 1: Average of Annual Average Treatment Effect on Treated

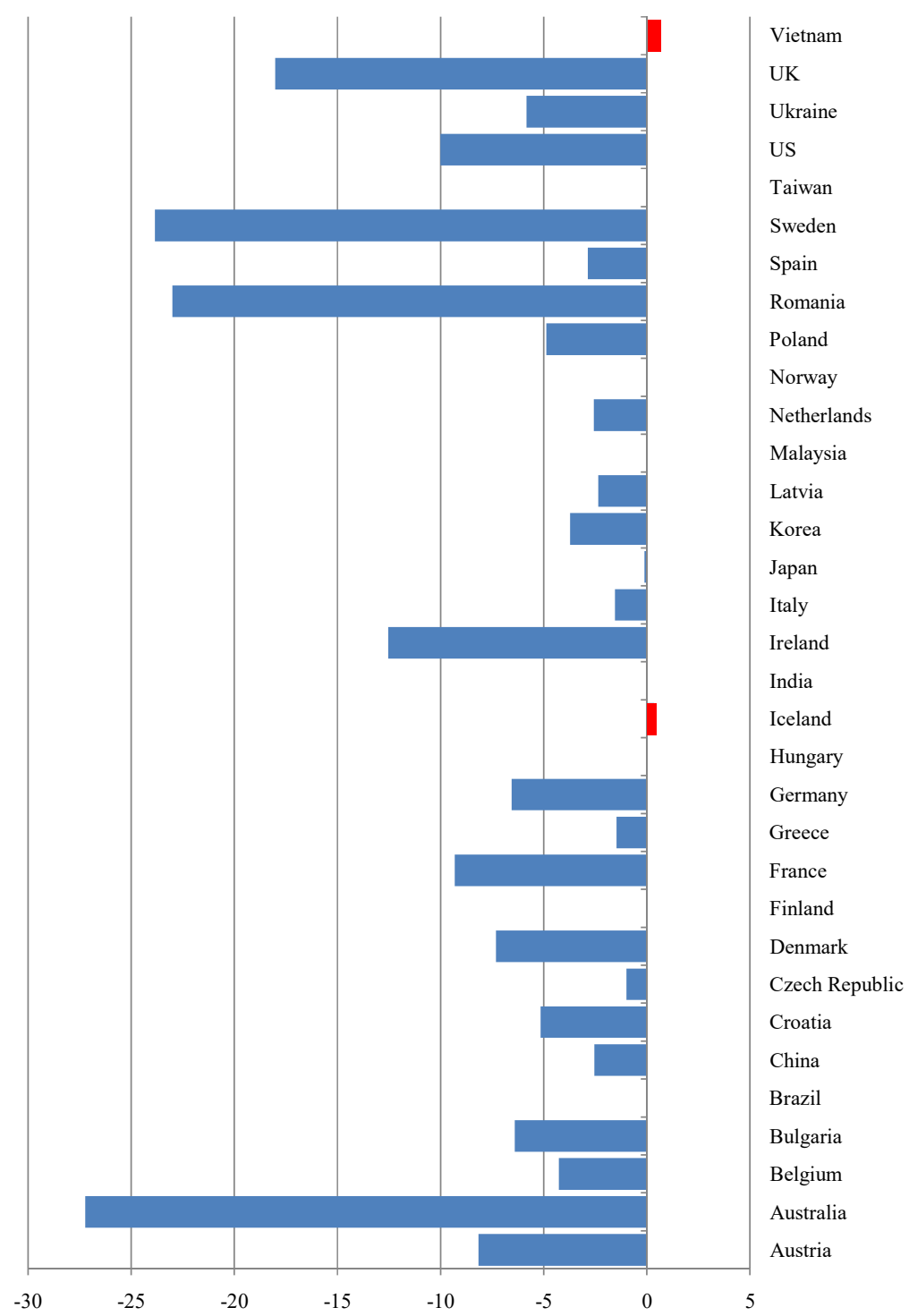


Figure 2: Effect of Listing on ROA based on Fixed Effect Panel Regressions

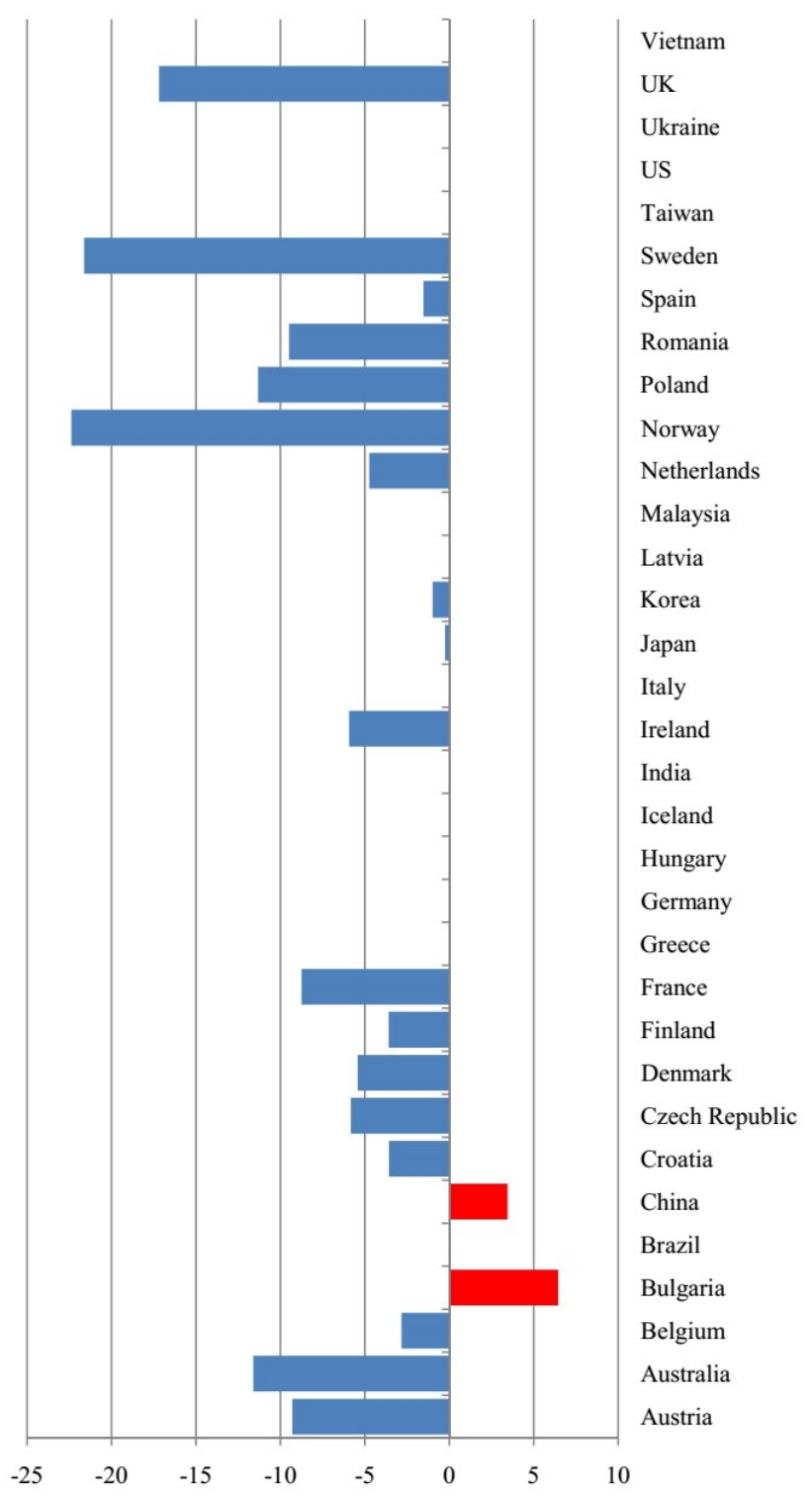


Table 1a: Number of Observations by Country

\begin{tabular}{|c|c|}
\hline Countries & Number of Observations \\
\hline Austria & 1337 \\
\hline Australia & 1192 \\
\hline Belgium & 51852 \\
\hline Bulgaria & 75667 \\
\hline Brazil & 69 \\
\hline China & 279384 \\
\hline Croatia & 41054 \\
\hline Czech Republic & 60701 \\
\hline Denmark & 1536 \\
\hline Finland & 22748 \\
\hline France & 62809 \\
\hline Greece & 22722 \\
\hline Germany & 34162 \\
\hline Hungary & 93164 \\
\hline Iceland & 1672 \\
\hline India & 1428 \\
\hline Ireland & 1786 \\
\hline Italy & 425534 \\
\hline Japan & 132846 \\
\hline Korea & 109704 \\
\hline Latvia & 15685 \\
\hline Malaysia & 357 \\
\hline Netherlands & 1484 \\
\hline Norway & 2980 \\
\hline Poland & 14116 \\
\hline Romania & 123180 \\
\hline Spain & 317753 \\
\hline Sweden & 35217 \\
\hline Taiwan & 5298 \\
\hline US & 7607 \\
\hline Ukraine & 110105 \\
\hline UK & 18602 \\
\hline Vietnam & 28038 \\
\hline
\end{tabular}


Table 1b: Country Level Variables Descriptions

\begin{tabular}{|c|c|c|c|c|c|c|c|}
\hline & Variable & Sources & Obs & Mean & Std. Dev. & Min & Max \\
\hline \multirow{4}{*}{ Corporate Governance } & Self Dealing Index & Djankov et al. 2008 & 320 & 0.447 & 0.22 & 0.081 & 0.95 \\
\hline & Anti-director Rights Index & Holger Spamann, 2006 & 210 & 3.976 & 2.185 & 2 & 14 \\
\hline & Protection of minority shareholders (1-7(best)) & World Economic Forum & 320 & 4.579 & 0.782 & 2.704 & 6.209 \\
\hline & Corporate Board Efficacy (1-7(Best)) & World Economic Forum & 320 & 4.945 & 0.672 & 3.725 & 6.272 \\
\hline \multirow{3}{*}{ Creditor's Rights } & Creditor's Rights & Djankov et al. 2008 & 320 & 2.031 & 0.919 & 0 & 4 \\
\hline & Time Resolve Insolvency (Years) & World Bank, Doing Business Project & 320 & 1.788 & 1.248 & 0 & 6.5 \\
\hline & Strength of Insolvency Framework (0-7(Strong)) & World Bank & 330 & 64.707 & 28.667 & 9.48 & 99.92 \\
\hline \multirow{3}{*}{ Institutional Quality } & Property Rights (1-7(Best)) & World Economic Forum & 320 & 5.019 & 0.977 & 2.511 & 6.638 \\
\hline & Rule of Law (-2.5 to 2.5$)$ & World Bank & 330 & 0.977 & 0.819 & -0.819 & 2.1 \\
\hline & Trust in People & World Value Survey & 240 & 33.985 & 17.714 & 8.15 & 73.7 \\
\hline Financial Development & Credit to GDP Ratio (Credit to Pvt. Sector) & World Bank & 318 & 101.157 & 43.56 & 26.51 & 201.259 \\
\hline \multirow{3}{*}{$\begin{array}{l}\text { Product Market } \\
\text { Competition }\end{array}$} & $\begin{array}{l}\text { New Business Registered (new registrations per 1,000 } \\
\text { people ages 15-64) }\end{array}$ & World Bank & 243 & 4.466 & 3.667 & 0.065 & 15.742 \\
\hline & Trade Barriers (1-7(Best)) & World Economic Forum & 320 & 4.695 & 0.579 & 3.336 & 6.168 \\
\hline & Cost of starting a Business(\% of income per capita) & World Bank & 315 & 5.263 & 5.831 & 0 & 41.5 \\
\hline \multirow{3}{*}{ Macro Volatility } & $\begin{array}{l}\text { Std. Deviation of Inflation from Last } 5 \text { Years Average } \\
\text { Inflation } \\
\text { Std. Deviation of GDP growth from Last } 5 \text { Year }\end{array}$ & Own Estimation Using World Bank data & 330 & 1.81 & 2.208 & 0.235 & 20.167 \\
\hline & $\begin{array}{l}\text { Std. Deviation of GDP growth from Last } 5 \text { Year } \\
\text { Average GDP Growth }\end{array}$ & Own Estimation Using World Bank data & 330 & 2.608 & 2.004 & 0.212 & 11.54 \\
\hline & Coefficient of variation of exchange rate & Own Estimation Using World Bank data & 326 & 4.981 & 5.452 & 0.654 & 41.91 \\
\hline Inflation Rate & Inflation (CPI Inflation) & World Bank & 330 & 2.83 & 4.264 & -1.676 & 48.684 \\
\hline Interest Rate* & Interest Rate & Datastream, CEIC & 319 & 5.721 & 8.004 & -0.695 & 52.1 \\
\hline
\end{tabular}

*: 3 Month Average T-Bill Rate, PLR or 3 Month Avg. Lending Rate 
Table 1c (Part 1): Correlation among the Country-level Variables

\begin{tabular}{|c|c|c|c|c|c|c|c|c|c|c|c|c|c|c|}
\hline & & [1] & {$[2]$} & [3] & [4] & [5] & [6] & [7] & {$[8]$} & [9] & {$[10]$} & [11] & [12] & [13] \\
\hline $\begin{array}{l}\text { Average Treatment Effect on Treated (Effect on ROA } \\
\text { due to Listing) }\end{array}$ & {$[1]$} & 1 & & & & & & & & & & & & \\
\hline Self Dealing Index & [2] & -0.053 & 1 & & & & & & & & & & & \\
\hline Corporate Goveranace Quality Index & [3] & -0.132 & 0.427 & 1 & & & & & & & & & & \\
\hline Anti-director Rights Index & [4] & 0.101 & 0.200 & 0.476 & 1 & & & & & & & & & \\
\hline Protection of minority shareholders (1-7(best)) & [5] & -0.327 & 0.208 & 0.140 & -0.128 & 1 & & & & & & & & \\
\hline Corporate Board Efficacy (1-7(Best)) & [6] & -0.405 & 0.118 & 0.038 & -0.205 & 0.875 & 1 & & & & & & & \\
\hline Extent of Director's Liability (1-10(Best)) & [7] & 0.268 & 0.262 & 0.189 & 0.552 & -0.042 & -0.147 & 1 & & & & & & \\
\hline Strength of Legal Rights(1-12(Strong)) & [8] & -0.316 & 0.608 & 0.135 & -0.135 & 0.509 & 0.546 & -0.224 & 1 & & & & & \\
\hline Creditor's Rights & [9] & -0.070 & 0.577 & 0.412 & 0.101 & 0.007 & -0.050 & 0.266 & 0.428 & 1 & & & & \\
\hline Time Resolve Insolvency (Years) & {$[10]$} & 0.244 & -0.225 & -0.182 & 0.436 & -0.459 & -0.547 & 0.055 & -0.378 & -0.330 & 1 & & & \\
\hline Strength of Insolvency Framework (0-7(Strong)) & [11] & -0.354 & -0.024 & 0.248 & -0.427 & 0.509 & 0.528 & -0.263 & 0.373 & 0.296 & -0.859 & 1 & & \\
\hline Property Rights (1-7(Best)) & [12] & -0.350 & 0.103 & 0.263 & -0.289 & 0.856 & 0.875 & -0.208 & 0.475 & 0.052 & -0.709 & 0.739 & 1 & \\
\hline Rule of Law (-2.5 to 2.5$)$ & [13] & -0.473 & -0.097 & 0.203 & -0.400 & 0.702 & 0.786 & -0.396 & 0.455 & 0.011 & -0.724 & 0.859 & 0.909 & 1 \\
\hline Trust in People & [14] & -0.533 & -0.181 & -0.022 & -0.429 & 0.644 & 0.597 & -0.532 & 0.402 & -0.001 & -0.410 & 0.720 & 0.652 & 0.782 \\
\hline Credit to GDP Ratio (Credit to Pvt. Sector) & [15] & -0.433 & 0.348 & 0.285 & -0.095 & 0.413 & 0.464 & 0.059 & 0.387 & 0.273 & -0.713 & 0.645 & 0.535 & 0.573 \\
\hline $\begin{array}{l}\text { Sum of stock market capitalization, private bond market } \\
\text { capitalization and bank credit to pvt. Sector (\% of GDP) }\end{array}$ & & & & & & & & & & & & & & \\
\hline Market Capitalization (\% of GDP) & $\begin{array}{l}116] \\
{[17]}\end{array}$ & $\begin{array}{l}-0.135 \\
-0.172\end{array}$ & $\begin{array}{r}-0.153 \\
0.539\end{array}$ & $\begin{array}{l}0.453 \\
0.223\end{array}$ & $\begin{array}{l}-0.064 \\
-0.040\end{array}$ & $\begin{array}{l}0.031 \\
0.591\end{array}$ & $\begin{array}{l}0.232 \\
0.542\end{array}$ & $\begin{array}{r}-0.059 \\
0.126\end{array}$ & $\begin{array}{r}-0.197 \\
0.543\end{array}$ & $\begin{array}{r}-0.066 \\
0.118\end{array}$ & $\begin{array}{r}-0.459 \\
-0.305\end{array}$ & $\begin{array}{l}0.365 \\
0.201\end{array}$ & $\begin{array}{l}0.398 \\
0.525\end{array}$ & $\begin{array}{l}0.447 \\
0.372\end{array}$ \\
\hline Prevalence of Foreign Ownership(1-7(Best)) & [18] & -0.405 & 0.343 & 0.384 & -0.013 & 0.657 & 0.773 & -0.071 & 0.621 & 0.182 & -0.567 & 0.507 & 0.766 & 0.728 \\
\hline New Business Registered (new registrations per 1,000 & & & & & & & & & & & & & & \\
\hline people ages $15-64)$ & [19] & -0.584 & 0.418 & 0.440 & 0.037 & 0.375 & 0.452 & -0.327 & 0.648 & 0.387 & -0.456 & 0.540 & 0.470 & 0.576 \\
\hline Trade Barriers (1-7(Best)) & {$[20]$} & -0.140 & 0.360 & 0.112 & -0.336 & 0.655 & 0.543 & -0.108 & 0.478 & 0.163 & -0.563 & 0.514 & 0.622 & 0.501 \\
\hline Cost of starting a Business( $\%$ of income per capita) & [21] & 0.368 & 0.071 & -0.191 & -0.041 & -0.473 & -0.620 & -0.069 & -0.247 & 0.164 & 0.516 & -0.499 & -0.597 & -0.677 \\
\hline $\begin{array}{l}\text { Std. Deviation of Inflation from Last } 5 \text { Years Average } \\
\text { Inflation } \\
\text { Std. Deviation of GDP growth from Last } 5 \text { Year }\end{array}$ & {$[22]$} & 0.432 & -0.081 & -0.039 & 0.188 & -0.468 & -0.591 & 0.138 & -0.293 & -0.077 & 0.555 & -0.419 & -0.610 & -0.575 \\
\hline $\begin{array}{l}\text { Sta. Devlation of GDP growth from Last } 5 \text { Year } \\
\text { Average GDP Growth }\end{array}$ & {$[23]$} & 0.221 & -0.317 & -0.041 & 0.189 & -0.070 & -0.243 & 0.161 & -0.296 & -0.171 & 0.177 & -0.052 & -0.103 & -0.119 \\
\hline Coefficient of variation of exchange rate & [24] & -0.055 & 0.028 & -0.148 & 0.522 & -0.038 & -0.094 & 0.117 & 0.000 & -0.040 & 0.538 & -0.448 & -0.289 & -0.367 \\
\hline Inflation (CPI Inflation) & {$[25]$} & 0.174 & 0.074 & -0.038 & 0.642 & -0.114 & -0.274 & 0.206 & -0.133 & 0.010 & 0.711 & -0.645 & -0.413 & -0.569 \\
\hline Interest Rate & {$[26]$} & 0.186 & -0.120 & -0.111 & 0.755 & -0.324 & -0.350 & 0.342 & -0.322 & -0.148 & 0.660 & -0.660 & -0.532 & -0.606 \\
\hline
\end{tabular}


Table 1c (Part 2): Correlation among the Country-level Variables

\begin{tabular}{|c|c|c|c|c|c|c|c|c|c|c|c|c|c|c|}
\hline & & [14] & [15] & [16] & [17] & [18] & [19] & [20] & {$[21]$} & {$[22]$} & {$[23]$} & {$[24]$} & {$[25]$} & [26] \\
\hline Trust in People & [14] & 1 & & & & & & & & & & & & \\
\hline Credit to GDP Ratio (Credit to Pvt. Sector) & [15] & 0.358 & 1 & & & & & & & & & & & \\
\hline $\begin{array}{l}\text { Sum of stock market capitalization, private bond market } \\
\text { capitalization and bank credit to pvt. Sector (\% of GDP) }\end{array}$ & [16] & -0.013 & 0267 & 1 & & & & & & & & & & \\
\hline Market Capitalization (\% of GDP) & [17] & 0.190 & $\begin{array}{l}0.207 \\
0.576\end{array}$ & 0.017 & 1 & & & & & & & & & \\
\hline Prevalence of Foreign Ownership(1-7(Best)) & [18] & 0.337 & 0.658 & 0.431 & 0.653 & 1 & & & & & & & & \\
\hline New Business Registered (new registrations per 1,000 & & & & & & & & & & & & & & \\
\hline people ages $15-64)$ & [19] & 0.558 & 0.644 & 0.194 & 0.398 & 0.614 & 1 & & & & & & & \\
\hline Trade Barriers (1-7(Best)) & [20] & 0.472 & 0.395 & 0.118 & 0.572 & 0.439 & 0.410 & 1 & & & & & & \\
\hline Cost of starting a Business $(\%$ of income per capita) & [21] & -0.385 & -0.533 & -0.488 & -0.337 & -0.642 & -0.482 & -0.287 & 1 & & & & & \\
\hline $\begin{array}{l}\text { Std. Deviation of Inflation from Last } 5 \text { Years Average } \\
\text { Inflation }\end{array}$ & [22] & -0.326 & -0.507 & -0.348 & -0.377 & -0.541 & -0.446 & -0.368 & 0.409 & 1 & & & & \\
\hline $\begin{array}{l}\text { Std. Deviation of GDP growth from Last } 5 \text { Year } \\
\text { Average GDP Growth }\end{array}$ & [23] & -0.044 & -0.169 & -0.069 & -0.090 & -0.226 & -0.271 & -0.042 & 0.022 & 0.049 & 1 & & & \\
\hline Coefficient of variation of exchange rate & [24] & -0.059 & -0.168 & -0.578 & -0.159 & -0.177 & -0.025 & -0.437 & 0.205 & 0.192 & 0.033 & 1 & & \\
\hline Inflation (CPI Inflation) & [25] & -0.292 & -0.436 & -0.527 & -0.211 & -0.286 & -0.223 & -0.397 & 0.450 & 0.305 & 0.143 & 0.806 & 1 & \\
\hline Interest Rate & [26] & -0.405 & -0.446 & -0.374 & -0.365 & -0.421 & -0.236 & -0.530 & 0.167 & 0.269 & 0.226 & 0.736 & 0.794 & 1 \\
\hline
\end{tabular}


Table 2: Year-wise ATT

\begin{tabular}{|c|c|c|c|c|c|c|c|c|c|c|}
\hline Countries & בATT2008 & בATT2009 & בATT2010 & בATT2011 & בATT2012 & בATT2013 & בATT2014 & בATT2015 & בATT2016 & ATT2017 \\
\hline Austria & & -12.614 & & -8.387 & -8.873 & -8.942 & -8.104 & 0 & -10.270 & \\
\hline Australia & -28.568 & -28.414 & -30.392 & -29.452 & -27.908 & -28.145 & -23.989 & -27.387 & -23.029 & -25.047 \\
\hline Belgium & -4.121 & -3.468 & -4.008 & -4.097 & -3.877 & -4.076 & -6.273 & -4.299 & -4.171 & \\
\hline Brazil & & & & & & & 0 & 0 & 0 & \\
\hline China & -0.978 & -1.379 & 0 & -2.222 & -2.542 & -3.680 & -1.837 & -4.962 & -5.355 & \\
\hline Croatia & -4.745 & -4.477 & -4.821 & -4.436 & -4.575 & -5.816 & -5.681 & -5.973 & -5.775 & \\
\hline Finland & 0 & 0 & 0 & 0 & 0 & 0 & 0 & 0 & 0 & 0 \\
\hline France & -9.529 & -8.924 & -9.049 & -8.408 & -8.297 & -8.890 & -9.982 & -8.240 & -9.130 & -12.710 \\
\hline Greece & -1.048 & -1.022 & -1.721 & -1.572 & -1.740 & -1.732 & -1.732 & -2.097 & -1.995 & 0 \\
\hline Germany & -6.692 & -6.627 & -6.367 & -6.688 & -6.601 & -6.801 & -6.384 & -6.589 & -6.905 & -5.919 \\
\hline Hungary & 0 & 0 & 0 & 0 & 0 & 0 & 0 & 0 & 0 & \\
\hline Japan & 0 & 0 & 0 & -0.544 & 0 & 0 & 0.000 & 0 & 0 & -0.623 \\
\hline Korea & -4.769 & -3.930 & -4.527 & -4.218 & -3.563 & -3.678 & -3.150 & -3.068 & -3.472 & -2.789 \\
\hline Latvia & 0 & 0 & 0 & -7.260 & 0 & -7.914 & 0 & -5.999 & 0 & \\
\hline Malaysia & 0 & 0 & 0 & 0 & 0 & 0 & 0 & 0 & 0 & 0 \\
\hline Netherlands & 0 & -10.726 & -6.638 & -8.320 & 0 & 0 & 0 & 0 & 0 & 0 \\
\hline Norway & & & & & & & & -85.823 & 0 & \\
\hline Poland & -3.739 & -4.882 & -5.573 & -5.376 & -5.119 & -5.110 & -4.388 & -5.740 & -3.913 & \\
\hline Romania & -21.056 & -25.229 & -24.506 & -23.557 & -21.876 & -27.109 & -26.871 & -24.401 & -12.454 & \\
\hline Spain & -3.003 & -3.267 & -3.915 & -2.889 & -3.140 & -3.604 & -2.781 & -2.781 & -3.219 & 0 \\
\hline Sweden & -23.536 & -23.554 & -23.850 & -23.532 & -24.176 & -23.388 & -24.134 & -24.417 & -24.594 & -23.385 \\
\hline Taiwan & 0 & 0.000 & 0 & 0 & 0 & 0 & 0 & 0 & 0 & 0 \\
\hline US & -16.615 & 0.000 & -17.709 & -9.785 & -5.957 & & & & & \\
\hline
\end{tabular}

Note: The non-zero values are the ATT values which were significant at 10 percent level of significance.

For all those years in which ATT takes the value zero are the statistically insignificant values.

In the cases where the ATT was not estimated, we put that value as missing. 
Table 3a: Results-Changing the Corporate Governance Indicators

\begin{tabular}{|c|c|c|c|c|c|c|}
\hline & {$[1]$} & [2] & [3] & [4] & [5] & [6] \\
\hline Self Dealing Index & $\begin{array}{c}-76.470^{* * *} \\
(-11.540)\end{array}$ & & & & & \\
\hline Corporate Governance Quality Index & & $\begin{array}{c}-4.603 * * * \\
(-11.540)\end{array}$ & & & & \\
\hline Anti-director Rights Index & & & $\begin{array}{c}0.107 \\
(0.180)\end{array}$ & & & \\
\hline Protection of minority shareholders interests & & & & $\begin{array}{c}1.939 \\
(1.480)\end{array}$ & & \\
\hline Corporate Board Efficacy & & & & & $\begin{array}{r}2.361^{* *} \\
(2.180)\end{array}$ & \\
\hline Extent of Director's Liability & & & & & & $\begin{array}{r}0.0001 \\
(0.00)\end{array}$ \\
\hline Creditor's Rights & $\begin{array}{c}23.450^{* * * *} \\
(13.710)\end{array}$ & $\begin{array}{c}53.740^{* * *} \\
(14.760)\end{array}$ & $\begin{array}{c}9.334^{* *} \\
(3.020)\end{array}$ & $\begin{array}{c}-30.950^{* * *} \\
(-6.280)\end{array}$ & $\begin{array}{c}-27.930^{* * *} \\
(-6.550)\end{array}$ & $\begin{array}{c}-28.220^{* * *} \\
(-6.790)\end{array}$ \\
\hline Property Rights & $\begin{array}{c}0.267 \\
(0.410)\end{array}$ & $\begin{array}{c}0.267 \\
(0.410)\end{array}$ & $\begin{array}{c}-0.278 \\
(-0.250)\end{array}$ & $\begin{array}{c}-0.481 \\
(-0.560)\end{array}$ & $\begin{array}{c}-0.472 \\
(-0.630)\end{array}$ & $\begin{array}{c}0.268 \\
(0.400)\end{array}$ \\
\hline Credit to GDP Ratio & $\begin{array}{c}0.022 \\
(0.800)\end{array}$ & $\begin{array}{c}0.022 \\
(0.800)\end{array}$ & $\begin{array}{c}0.033 \\
(1.130)\end{array}$ & $\begin{array}{c}0.017 \\
(0.680)\end{array}$ & $\begin{array}{c}0.034 \\
(1.270)\end{array}$ & $\begin{array}{c}0.022 \\
(0.800)\end{array}$ \\
\hline New Business Registered & $\begin{array}{l}0.333^{*} \\
(1.890)\end{array}$ & $\begin{array}{l}0.333^{*} \\
(1.890)\end{array}$ & $\begin{array}{c}0.586^{* *} \\
(2.230)\end{array}$ & $\begin{array}{l}0.341^{*} \\
(1.850)\end{array}$ & $\begin{array}{c}0.251 \\
(1.480)\end{array}$ & $\begin{array}{l}0.333^{*} \\
(1.880)\end{array}$ \\
\hline Std Deviation Inflation & $\begin{array}{l}-0.017 \\
(-0.130)\end{array}$ & $\begin{array}{c}-0.017 \\
(-0.130)\end{array}$ & $\begin{array}{l}-1.727 \\
(-1.570)\end{array}$ & $\begin{array}{l}-0.006 \\
(-0.040)\end{array}$ & $\begin{array}{c}0.103 \\
(0.850)\end{array}$ & $\begin{array}{l}-0.017 \\
(-0.130)\end{array}$ \\
\hline Inflation (CPI Inflation) & $\begin{array}{c}0.011 \\
(0.290)\end{array}$ & $\begin{array}{c}0.011 \\
(0.290)\end{array}$ & $\begin{array}{c}0.065 \\
(0.340)\end{array}$ & $\begin{array}{c}0.012 \\
(0.300)\end{array}$ & $\begin{array}{c}0.001 \\
(0.040)\end{array}$ & $\begin{array}{c}0.011 \\
(0.290)\end{array}$ \\
\hline Interest Rate & $\begin{array}{c}-0.063 \\
(-0.340)\end{array}$ & $\begin{array}{c}-0.063 \\
(-0.340)\end{array}$ & $\begin{array}{c}0.137 \\
(0.700)\end{array}$ & $\begin{array}{c}-0.138 \\
(-0.760)\end{array}$ & $\begin{array}{c}-0.030 \\
(-0.150)\end{array}$ & $\begin{array}{c}-0.063 \\
(-0.340)\end{array}$ \\
\hline Constant & $\begin{array}{c}-48.070^{* * *} \\
(-11.620) \\
\end{array}$ & $\begin{array}{c}116.900^{* * * *} \\
(8.170) \\
\end{array}$ & $\begin{array}{c}-65.470 * * * \\
(-5.650) \\
\end{array}$ & $\begin{array}{c}52.560 * * * \\
(5.560) \\
\end{array}$ & $\begin{array}{c}38.130^{* * *} \\
(3.520) \\
\end{array}$ & $\begin{array}{c}49.050^{* * *} \\
(5.540) \\
\end{array}$ \\
\hline Observations & 195 & 195 & 127 & 195 & 195 & 195 \\
\hline Adjusted R-squared & 0.921 & 0.921 & 0.935 & 0.922 & 0.926 & 0.921 \\
\hline
\end{tabular}

$\mathrm{t}$ statistics in parentheses

*denotes significance at $10 \%, * *$ at $5 \%$ and $* * *$ at $1 \%$ 
Table 3b: Results-Changing the Creditor's Rights Indicator

\begin{tabular}{|c|c|c|c|c|}
\hline & [1] & [7] & [8] & [9] \\
\hline Self Dealing Index & $\begin{array}{c}-76.470^{* * *} \\
(-11.540)\end{array}$ & $\begin{array}{c}-41.040^{* * *} \\
(-6.820)\end{array}$ & $\begin{array}{c}-45.930^{* *} \\
(-2.550)\end{array}$ & $\begin{array}{c}-42.570^{* * * *} \\
(-6.590)\end{array}$ \\
\hline Creditor's Rights & $\begin{array}{c}23.450 * * * \\
(13.710)\end{array}$ & & & \\
\hline Strength of Legal Rights & & $\begin{array}{l}0.204 * \\
(1.870)\end{array}$ & & \\
\hline Strength of Insolvency Framework & & & $\begin{array}{l}-1.635^{* *} \\
(-2.120)\end{array}$ & \\
\hline Time Resolve Insolvency & & & & $\begin{array}{c}0.015 \\
(0.580)\end{array}$ \\
\hline Property Rights & $\begin{array}{c}0.267 \\
(0.410)\end{array}$ & $\begin{array}{c}0.297 \\
(0.470)\end{array}$ & $\begin{array}{c}1.042 \\
(0.590)\end{array}$ & $\begin{array}{c}0.285 \\
(0.450)\end{array}$ \\
\hline Credit to GDP Ratio & $\begin{array}{c}0.022 \\
(0.800)\end{array}$ & $\begin{array}{c}0.022 \\
(0.980)\end{array}$ & $\begin{array}{c}0.014 \\
(0.250)\end{array}$ & $\begin{array}{c}0.021 \\
(0.970)\end{array}$ \\
\hline New Business Registered & $\begin{array}{c}0.258^{*} \\
(1.89)\end{array}$ & $\begin{array}{l}-0.557^{*} \\
(1.680)\end{array}$ & $\begin{array}{c}0.333 \\
(-0.760)\end{array}$ & $\begin{array}{c}0.275 \\
(1.530)\end{array}$ \\
\hline Std. Deviation Inflation & $\begin{array}{c}-0.017 \\
(-0.130)\end{array}$ & $\begin{array}{c}-0.019 \\
(-0.140)\end{array}$ & $\begin{array}{c}0.139 \\
(0.850)\end{array}$ & $\begin{array}{c}-0.007 \\
(-0.050)\end{array}$ \\
\hline Inflation & $\begin{array}{c}0.011 \\
(0.290)\end{array}$ & $\begin{array}{c}0.018 \\
(0.480)\end{array}$ & $\begin{array}{c}0.141^{* *} \\
(2.370)\end{array}$ & $\begin{array}{c}0.013 \\
(0.360)\end{array}$ \\
\hline Interest Rate & $\begin{array}{c}-0.063 \\
(-0.340)\end{array}$ & $\begin{array}{c}-0.113 \\
(-0.690)\end{array}$ & $\begin{array}{c}-1.815^{* *} \\
(-2.320)\end{array}$ & $\begin{array}{c}-0.097 \\
(-0.570)\end{array}$ \\
\hline Constant & $\begin{array}{c}-48.070^{* * *} \\
(-11.620) \\
\end{array}$ & $\begin{array}{c}-3.798 \\
(-1.580) \\
\end{array}$ & $\begin{array}{c}37.970 * * \\
(2.080) \\
\end{array}$ & $\begin{array}{c}-3.517 \\
(-1.450) \\
\end{array}$ \\
\hline Observations & 195 & 202 & 86 & 202 \\
\hline Adjusted R-squared & 0.921 & 0.922 & 0.917 & 0.922 \\
\hline
\end{tabular}

$\mathrm{t}$ statistics in parentheses

$* \mathrm{p}<0.05, * * \mathrm{p}<0.01, * * * \mathrm{p}<0.001$ 
Table 3c: Results-Changing the Institutional Quality Indicators

\begin{tabular}{lccc}
\hline \hline & {$[1]$} & {$[10]$} & {$[11]$} \\
\hline Self Dealing Index & $-76.470^{* * *}$ & $-79.640^{* * *}$ & $-86.330^{* * *}$ \\
& $(-11.540)$ & $(-4.660)$ & $(-6.410)$ \\
Creditor's Rights & $23.450^{* * *}$ & $24.310^{* * *}$ & $27.960^{* * *}$ \\
& $(13.710)$ & $(6.760)$ & $(4.350)$ \\
Property Rights & 0.267 & & \\
& $(0.410)$ & & \\
Rule of Law & & 0.956 & \\
& & $(0.280)$ & 0.085 \\
Trust in People & & & $(0.560)$ \\
& & & 0.005 \\
Credit to GDP Ratio & 0.022 & 0.021 & $(0.430)$ \\
& $(0.800)$ & $(0.680)$ & $0.463^{* *}$ \\
New Business Registered & $0.333^{*}$ & $0.326 *$ & $(2.930)$ \\
Std. Deviation Inflation & $(1.89)$ & $(1.660)$ & 0.056 \\
Inflation & -0.017 & -0.022 & $(0.550)$ \\
& $(-0.130)$ & $(-0.160)$ & 0.015 \\
Interest Rate & 0.011 & 0.013 & $(0.660)$ \\
Constant & $(0.290)$ & $(0.400)$ & -0.120 \\
& -0.063 & -0.043 & $(-0.730)$ \\
Observations & $(-0.340)$ & $(-0.270)$ & $-56.160^{* *}$ \\
Adjusted R-squared & $-48.070^{* * *}$ & $-48.280^{* * *}$ & $(-3.370)$ \\
\hline
\end{tabular}

$\mathrm{t}$ statistics in parentheses

$*$ denotes significance at $10 \%,{ }^{* *}$ at $5 \%$ and $* * *$ at $1 \%$ 


\begin{tabular}{|c|c|c|c|c|}
\hline & {$[1]$} & {$[12]$} & {$[13]$} & {$[14]$} \\
\hline Self Dealing Index & $\begin{array}{c}-76.470^{* * *} \\
(-11.54)\end{array}$ & $\begin{array}{c}-75.680^{* * *} \\
(-14.280)\end{array}$ & $\begin{array}{c}-70.850^{* * *} \\
(-9.810)\end{array}$ & $\begin{array}{c}-72.130 * * * \\
(-11.120)\end{array}$ \\
\hline Creditor's Rights & $\begin{array}{c}23.450^{* * *} \\
(13.710)\end{array}$ & $\begin{array}{c}26.100 * * * \\
(12.090)\end{array}$ & $\begin{array}{c}23.870^{* * *} \\
(11.880)\end{array}$ & $\begin{array}{c}23.500^{* * *} \\
(14.320)\end{array}$ \\
\hline Property Rights & $\begin{array}{c}0.267 \\
(0.410)\end{array}$ & $\begin{array}{c}-0.417 \\
(-0.880)\end{array}$ & $\begin{array}{c}0.404 \\
(0.520)\end{array}$ & $\begin{array}{l}-0.175 \\
(-0.270)\end{array}$ \\
\hline Credit to GDP Ratio & $\begin{array}{c}0.022 \\
(0.800)\end{array}$ & & & \\
\hline $\begin{array}{l}\text { Sum of stock market capitalization and private bond } \\
\text { market capitalization and bank credit over GDP }\end{array}$ & & $\begin{array}{c}-0.574 \\
(-0.760)\end{array}$ & & \\
\hline Market Capitalization ( $\%$ of GDP) & & & $\begin{array}{l}-0.010 \\
(-0.630)\end{array}$ & \\
\hline Prevalence of Foreign Ownership & & & & $\begin{array}{l}1.175^{* *} \\
(1.970)\end{array}$ \\
\hline New Business Registered & $\begin{array}{l}0.333^{*} \\
(1.890)\end{array}$ & $\begin{array}{l}0.482 * * \\
(2.830)\end{array}$ & $\begin{array}{c}0.173 \\
(0.960)\end{array}$ & $\begin{array}{c}0.179 \\
(1.020)\end{array}$ \\
\hline Std. Deviation Inflation & $\begin{array}{c}-0.017 \\
(-0.130)\end{array}$ & $\begin{array}{l}-0.091 \\
(-0.930)\end{array}$ & $\begin{array}{c}-0.063 \\
(-0.130)\end{array}$ & $\begin{array}{c}0.005 \\
(0.040)\end{array}$ \\
\hline Inflation & $\begin{array}{c}0.011 \\
(0.290)\end{array}$ & $\begin{array}{c}0.030 \\
(0.910)\end{array}$ & $\begin{array}{l}-0.012 \\
(-0.110)\end{array}$ & $\begin{array}{c}0.003 \\
(0.070)\end{array}$ \\
\hline Interest Rate & $\begin{array}{c}-0.063 \\
(-0.340)\end{array}$ & $\begin{array}{c}0.085 \\
(0.680)\end{array}$ & $\begin{array}{c}0.053 \\
(0.290)\end{array}$ & $\begin{array}{c}0.031 \\
(0.170)\end{array}$ \\
\hline Constant & $\begin{array}{c}-48.070^{* * *} \\
(-11.620)\end{array}$ & $\begin{array}{c}-47.730 * * * \\
(-10.570)\end{array}$ & $\begin{array}{c}-49.330^{* * *} \\
(-11.640)\end{array}$ & $\begin{array}{c}-51.520 * * * \\
(-11.220) \\
\end{array}$ \\
\hline Observations & 195 & 181 & 190 & 197 \\
\hline Adjusted R-squared & 0.921 & 0.923 & 0.919 & 0.920 \\
\hline
\end{tabular}

$\mathrm{t}$ statistics in parenthese

$*$ denotes significance at $10 \%, * *$ at $5 \%$ and $* * *$ at $1 \%$ 
Table 3e: Results-Changing the Product Market Competition Indicators

\begin{tabular}{lccc}
\hline & {$[1]$} & {$[15]$} & {$[16]$} \\
\hline Self Dealing Index & $-76.470^{* * *}$ & $-69.260^{* * *}$ & $-68.980^{* * *}$ \\
& $(-11.540)$ & $(-12.100)$ & $(-11.920)$ \\
Creditor's Rights & $23.450^{* * *}$ & $21.720^{* * *}$ & $21.680^{* * *}$ \\
& $(13.710)$ & $(12.520)$ & $(12.560)$ \\
Property Rights & 0.267 & 0.721 & 0.746 \\
& $(0.410)$ & $(1.300)$ & $(1.530)$ \\
Credit to GDP Ratio & 0.0220 & 0.006 & 0.007 \\
& $(0.800)$ & $(0.270)$ & $(0.270)$ \\
New Business Registered & $0.333^{*}$ & & \\
& $(1.890)$ & & \\
Cost of starting a business & & -0.014 & \\
& & $(-0.790)$ & -0.079 \\
Trade Barriers & & & $(-0.130)$ \\
Std. Deviation Inflation & & -0.004 \\
Inflation & -0.017 & -0.011 & $(-0.030)$ \\
Interest Rate & $(-0.130)$ & $(-0.090)$ & 0.009 \\
Constant & 0.011 & 0.011 & $(0.280)$ \\
& $(0.290)$ & $(0.320)$ & -0.099 \\
Observations & -0.063 & -0.101 & $(-0.49)$ \\
Adjusted R-squared & $(-0.340)$ & $(-0.570)$ & $-44.180^{* * *}$ \\
\hline
\end{tabular}

$\mathrm{t}$ statistics in parentheses

*denotes significance at $10 \%, * *$ at $5 \%$ and $* * *$ at $1 \%$ 
Table 3f: Results-Changing the Macro-Volatility Indicators

\begin{tabular}{lccc}
\hline \hline & {$[1]$} & {$[17]$} & {$[18]$} \\
\hline Self Dealing Index & $-76.470^{* * *}$ & $-77.550^{* * *}$ & $-76.270^{* * *}$ \\
Creditor's Rights & $(-11.540)$ & $(-11.010)$ & $(-11.190)$ \\
& $23.450^{* * *}$ & $24.120^{* * *}$ & $23.370^{* * *}$ \\
Property Rights & $(13.710)$ & $(15.230)$ & $(14.220)$ \\
& 0.267 & 0.160 & 0.303 \\
Credit to GDP Ratio & $(0.410)$ & $(0.270)$ & $(0.480)$ \\
& 0.022 & 0.0220 & 0.022 \\
New Business Registered & $(0.800)$ & $(0.800)$ & $(0.820)$ \\
& $0.333^{*}$ & $0.329^{*}$ & $0.331^{*}$ \\
Std. Deviation Inflation & $(1.890)$ & $(1.910)$ & $(1.870)$ \\
& -0.017 & & \\
Std. Deviation GDP growth & $(-0.130)$ & & \\
Coefficient of variation of exchange rate & & -0.140 & \\
Inflation & & $(-0.860)$ & \\
& & & 0.009 \\
Interest Rate & & & $0.300)$ \\
Constant & 0.011 & -0.003 & 0.005 \\
& $(0.290)$ & $(-0.090)$ & $(0.180)$ \\
\hline Observations & -0.063 & -0.022 & -0.066 \\
Adjusted R-squared & $(-0.340)$ & $(-0.120)$ & $(-0.360)$ \\
\hline tstisics & $-48.070^{* * *}$ & $-48.730^{* * *}$ & $-48.240^{* * *}$ \\
& $(-11.620)$ & $(-11.510)$ & $(-10.810)$ \\
\hline
\end{tabular}

$\mathrm{t}$ statistics in parentheses

$*$ denotes significance at $10 \%, * *$ at $5 \%$ and $* * *$ at $1 \%$ 


\section{Appendix A1. Countrywise Descriptive Statistics}

Table A1.a: Austria: Descriptive Statistics

\begin{tabular}{lccccc}
\hline \hline & Obs. Number & Mean & Std. Dev. & Min & Max \\
\hline Listing (dummy) & 1884 & 0.058 & 0.234 & 0 & 1 \\
ROA(EBIT/TA, \%) & 1879 & 4.095 & 123.324 & -5257.721 & 564.141 \\
ROK (EBIT/Fixed Asset, \%) & 1878 & 0.072 & 16.999 & -715.05 & 100.387 \\
ROA(P/L before tax, \%) & 1876 & 6.569 & 11.845 & -82.88 & 95.78 \\
ROK & 1875 & 47.198 & 379.757 & -4877.153 & 10203.679 \\
D/A(ratio) & 1884 & 0.572 & 0.225 & 0.008 & 0.997 \\
Age & 1884 & 42.327 & 44.979 & 1 & 413 \\
Total Assets & 1884 & 174897.143 & 1222311.431 & 55 & 18577708 \\
Number of Workers & 1884 & 575.721 & 3336.254 & 1 & 47186 \\
\hline
\end{tabular}

Austria: Correlation Table

\begin{tabular}{|c|c|c|c|c|c|c|c|c|c|}
\hline & (1) & $(2)$ & $\overline{(3)}$ & $(4)$ & $(5)$ & $(6)$ & $(7)$ & (8) & $\overline{(9)}$ \\
\hline (1) Listing (dummy) & 1 & & & & & & & & \\
\hline (2) ROA (EBIT/TA, \%) & -0.073 & 1 & & & & & & & \\
\hline (3) ROK (EBIT) & -0.027 & 0.290 & 1 & & & & & & \\
\hline (4) ROA (P/L before tax, \%) & -0.074 & 0.980 & 0.290 & 1 & & & & & \\
\hline (5) $\mathrm{ROK}$ & -0.027 & 0.288 & 1 & 0.292 & 1 & & & & \\
\hline (6) $\mathrm{D} / \mathrm{A}$ (ratio) & -0.079 & -0.188 & -0.096 & -0.243 & -0.101 & 1 & & & \\
\hline (7) Age & 0.154 & -0.073 & -0.046 & -0.079 & -0.047 & -0.031 & 1 & & \\
\hline (8) Total Assets & 0.455 & -0.009 & -0.010 & -0.014 & -0.010 & -0.023 & 0.031 & 1 & \\
\hline (9) Number of Workers & 0.545 & -0.02 & -0.013 & -0.027 & -0.014 & -0.009 & 0.046 & 0.972 & 1 \\
\hline
\end{tabular}


Table A1.b: Australia: Descriptive Statistics

\begin{tabular}{lccccc}
\hline \hline & Obs. Number & Mean & Std. Dev. & Min & Max \\
\hline Listing (dummy) & 1899 & 0.34 & 0.474 & 0 & 1 \\
ROA (EBIT/TA, \%) & 1887 & -1.718 & 44.946 & -1283.302 & 109.256 \\
ROK (EBIT) & 1877 & -1.529 & 21.63 & -447 & 268.667 \\
ROA (P/L before tax, \%) & 1853 & 1.297 & 20.559 & -96.66 & 86.53 \\
ROK & 1843 & -26.962 & 1382.121 & -18962.6 & 40842.16 \\
D/A (ratio) & 1899 & 0.431 & 0.243 & 0 & 0.992 \\
Age & 1899 & 27.967 & 24.318 & 1 & 157 \\
Total Assets & 1899 & 372374.535 & 1251179.143 & 248 & 11731606 \\
Number of Workers & 1899 & 819.735 & 2933.308 & 1 & 33868 \\
\hline
\end{tabular}

Australia: Correlation Table

\begin{tabular}{lccccccccc}
\hline \hline & $(1)$ & $(2)$ & $(3)$ & $(4)$ & $(5)$ & $(6)$ & $(7)$ & $(8)$ & $(9)$ \\
\hline (1) Listing (dummy) & 1 & & & & & & & & \\
(2) ROA (EBIT/TA, \%) & -0.353 & 1 & & & & & & \\
(3) ROK (EBIT) & -0.095 & 0.277 & 1 & & & & & & \\
(4) ROA (P/L before tax, \%) & -0.42 & 0.833 & 0.205 & 1 & & & & & \\
(5) ROK & -0.069 & 0.221 & 0.779 & 0.247 & 1 & & & & \\
(6) D/A (ratio) & -0.371 & 0.152 & 0.026 & 0.161 & 0.019 & 1 & & & \\
(7) Age & 0.055 & 0.165 & 0.03 & 0.185 & 0.017 & 0.039 & 1 & & \\
(8) Total Assets & 0.291 & 0.076 & 0.015 & 0.077 & 0.009 & 0.121 & 0.438 & 1 & \\
(9) Number of Workers & 0.257 & 0.092 & 0.015 & 0.098 & 0.009 & 0.12 & 0.412 & 0.852 & 1 \\
\hline
\end{tabular}


Table A1.c: Belgium: Descriptive Statistics

\begin{tabular}{lccccc}
\hline \hline & Obs. Number & Mean & Std. Dev. & Min & Max \\
\hline Listing (dummy) & 62780 & 0.004 & 0.063 & 0 & 1 \\
ROA (EBIT/TA, \%) & 62779 & 6.223 & 14.703 & -544.444 & 1161.017 \\
ROK(EBIT/FIXED ASSET, \%) & 62044 & 0.789 & 7.985 & -328 & 846 \\
ROA (P/L before tax, \%) & 62707 & 4.870 & 12.274 & -99.630 & 99.800 \\
ROK & 61980 & 72.336 & 840.683 & -33088.762 & 79532.570 \\
D/A (ratio) & 62780 & 0.597 & 0.250 & 0 & 1 \\
Age & 62780 & 20.808 & 15.601 & 1 & 238 \\
Total Assets & 62780 & 31957.819 & 1777117.338 & 4 & 258381000 \\
Number of Workers & 62780 & 48.322 & 1791.944 & 1 & 206633 \\
\hline
\end{tabular}

Belgium: Correlation Table

\begin{tabular}{|c|c|c|c|c|c|c|c|c|c|}
\hline & 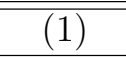 & 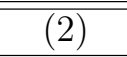 & $\overline{(3)}$ & $\overline{(4)}$ & $\overline{(5)}$ & $\overline{(6)}$ & $\overline{(7)}$ & $\overline{(8)}$ & $(9)$ \\
\hline (1) Listing (dummy) & 1 & & & & & & & & \\
\hline (2) ROA (EBIT/TA, \%) & -0.029 & 1 & & & & & & & \\
\hline (3) ROK (EBIT) & -0.008 & 0.218 & 1 & & & & & & \\
\hline (4) ROA (P/L before tax, \%) & -0.034 & 0.953 & 0.22 & 1 & & & & & \\
\hline (5) $\mathrm{ROK}$ & -0.007 & 0.193 & 0.904 & 0.215 & 1 & & & & \\
\hline (6) $\mathrm{D} / \mathrm{A}$ (ratio) & -0.011 & -0.083 & -0.033 & -0.174 & -0.047 & 1 & & & \\
\hline (7) Age & 0.16 & -0.128 & -0.026 & -0.1 & -0.017 & -0.281 & 1 & & \\
\hline (8) Total Assets & 0.249 & 0.002 & -0.001 & 0.001 & -0.001 & 0.003 & 0.029 & 1 & \\
\hline (9) Number of Workers & 0.314 & 0.001 & -0.002 & 0 & -0.002 & 0.004 & 0.054 & 0.978 & 1 \\
\hline
\end{tabular}


Table A1.d: Bulgaria: Descriptive Statistics

\begin{tabular}{lccccc}
\hline \hline & Obs. Number & Mean & Std. Dev. & Min & Max \\
\hline Listing (dummy) & 100618 & 0.002 & 0.047 & 0 & 1 \\
ROA (EBIT/TA, \%) & 100618 & 13.531 & 31.215 & -2866.667 & 2511.765 \\
ROK(EBIT/FIXED ASSET, \%) & 79579 & 2.009 & 13.443 & -1871 & 1472 \\
ROA (P/L before tax, \%) & 100068 & 12.265 & 21.355 & -100 & 100 \\
ROK & 79319 & 190.507 & 1154.933 & -18765.9 & 146819.766 \\
D/A (ratio) & 100618 & 0.407 & 0.312 & -0.02 & 1 \\
Age & 98609 & 10.171 & 8.408 & 1 & 168 \\
Total Assets & 100618 & 881.131 & 7057.107 & 1 & 790180 \\
Number of Workers & 100618 & 23.662 & 66.899 & 1 & 4543 \\
\hline
\end{tabular}

Bulgaria: Correlation Table

\begin{tabular}{|c|c|c|c|c|c|c|c|c|c|}
\hline & $\overline{(1)}$ & $\overline{(2)}$ & $\overline{(3)}$ & $\overline{(4)}$ & $\overline{(5)}$ & $\overline{(6)}$ & $\overline{(7)}$ & $\overline{(8)}$ & 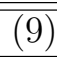 \\
\hline (1) Listing (dummy) & 1 & & & & & & & & \\
\hline (2) ROA (EBIT/TA, \%) & -0.028 & 1 & & & & & & & \\
\hline (3) ROK (EBIT) & -0.009 & 0.27 & 1 & & & & & & \\
\hline (4) ROA (P/L before tax, \%) & -0.028 & 0.98 & 0.273 & 1 & & & & & \\
\hline (5) $\mathrm{ROK}$ & -0.009 & 0.266 & 0.989 & 0.276 & 1 & & & & \\
\hline (6) $\mathrm{D} / \mathrm{A}$ (ratio) & -0.013 & -0.233 & -0.084 & -0.272 & -0.09 & 1 & & & \\
\hline (7) Age & 0.265 & -0.166 & -0.068 & -0.163 & -0.066 & -0.1 & 1 & & \\
\hline (8) Total Assets & 0.187 & -0.021 & -0.013 & -0.021 & -0.012 & 0.014 & 0.136 & 1 & \\
\hline (9) Number of Workers & 0.203 & 0.011 & -0.018 & 0.009 & -0.016 & 0.026 & 0.237 & 0.638 & 1 \\
\hline
\end{tabular}


Table A1.e: Brazil: Descriptive Statistics

\begin{tabular}{lccccc}
\hline \hline & Obs. Number & Mean & Std. Dev. & Min & Max \\
\hline Listing (dummy) & 1324 & 0.088 & 0.284 & 0 & 1 \\
ROA (EBIT/TA, \%) & 1324 & 3.228 & 21.091 & -425.869 & 73.538 \\
ROK(EBIT/FIXED ASSET, \%) & 1321 & 0.234 & 3.184 & -29 & 73.875 \\
ROA (P/L before tax, \%) & 1312 & 2.164 & 16.231 & -93.38 & 74.71 \\
ROK & 1309 & 25.941 & 341.145 & -2281.5 & 8327.443 \\
D/A (ratio) & 1324 & 0.512 & 0.259 & 0 & 0.996 \\
Age & 1324 & 33.548 & 18.763 & 1 & 125 \\
Total Assets & 1324 & 391558.443 & 2034492.625 & 8 & 26258178 \\
Number of Workers & 1324 & 1371.4 & 5204.178 & 2 & 120096 \\
\hline
\end{tabular}

Brazil: Correlation Table

\begin{tabular}{|c|c|c|c|c|c|c|c|c|c|}
\hline & $\overline{(1)}$ & 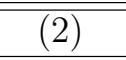 & 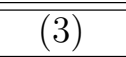 & $\overline{(4)}$ & 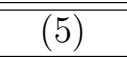 & $\overline{(6)}$ & $\overline{(7)}$ & $\overline{(8)}$ & (9) \\
\hline (1) Listing (dummy) & 1 & & & & & & & & \\
\hline (2) ROA (EBIT/TA, \%) & 0.088 & 1 & & & & & & & \\
\hline (3) ROK(EBIT/FIXED ASSET, \%) & -0.002 & 0.331 & 1 & & & & & & \\
\hline (4) ROA (P/L before tax, \%) & 0.08 & 0.88 & 0.315 & 1 & & & & & \\
\hline (5) $\mathrm{ROK}$ & -0.006 & 0.282 & 0.976 & 0.314 & 1 & & & & \\
\hline (6) $\mathrm{D} / \mathrm{A}$ (ratio) & 0.095 & -0.047 & -0.042 & -0.227 & -0.076 & 1 & & & \\
\hline (7) Age & 0.267 & 0.077 & -0.057 & 0.081 & -0.055 & 0.003 & 1 & & \\
\hline (8) Total Assets & 0.477 & 0.036 & -0.006 & 0.04 & -0.008 & 0.095 & 0.17 & 1 & \\
\hline (9) Number of Workers & 0.558 & 0.049 & -0.005 & 0.049 & -0.007 & 0.063 & 0.22 & 0.624 & 1 \\
\hline
\end{tabular}


Table A1.f: China: Descriptive Statistics

\begin{tabular}{lccccc}
\hline \hline & Obs. Number & Mean & Std. Dev. & Min & Max \\
\hline Listing (dummy) & 470805 & 0.043 & 0.204 & 0 & 1 \\
ROA (EBIT/TA, \%) & 470805 & 15.663 & 67.063 & -4508.403 & 19450.686 \\
ROK(EBIT/FIXED ASSET, \%) & 469995 & 0.774 & 12.393 & -1447.128 & 3375 \\
ROA (P/L before tax, \%) & 325392 & 9.416 & 18.266 & -100 & 100 \\
ROK & 324979 & 51.153 & 1472.016 & -99042.906 & 779355.625 \\
D/A (ratio) & 470805 & 0.542 & 0.282 & -46.571 & 1 \\
Age & 466439 & 8.24 & 5.673 & 1 & 116 \\
Total Assets & 470805 & 41279.528 & 471325.137 & -625 & 53719639 \\
Number of Workers & 470805 & 294.389 & 1800.102 & 1 & 200000 \\
\hline
\end{tabular}

China: Correlation Table

\begin{tabular}{|c|c|c|c|c|c|c|c|c|c|}
\hline & $\overline{(1)}$ & $\overline{(2)}$ & $\overline{(3)}$ & $\overline{(4)}$ & $\overline{(5)}$ & (6) & $\overline{(7)}$ & $\overline{(8)}$ & $\overline{(9)}$ \\
\hline (1) Listing (dummy) & 1 & & & & & & & & \\
\hline (2) ROA (EBIT/TA, \%) & -0.064 & 1 & & & & & & & \\
\hline (3) ROK(EBIT/FIXED ASSET, \%) & -0.01 & 0.147 & 1 & & & & & & \\
\hline (4) ROA (P/L before tax, \%) & -0.045 & 0.953 & 0.143 & 1 & & & & & \\
\hline (5) $\mathrm{ROK}$ & 0.004 & 0.051 & 0.381 & 0.058 & 1 & & & & \\
\hline (6) $\mathrm{D} / \mathrm{A}$ (ratio) & -0.118 & -0.202 & -0.014 & -0.225 & -0.011 & 1 & & & \\
\hline (7) Age & 0.251 & -0.081 & -0.012 & -0.077 & -0.001 & -0.032 & 1 & & \\
\hline (8) Total Assets & 0.247 & -0.024 & -0.005 & -0.02 & -0.001 & 0.011 & 0.122 & 1 & \\
\hline (9) Number of Workers & 0.274 & -0.023 & -0.006 & -0.018 & -0.002 & 0.002 & 0.139 & 0.765 & 1 \\
\hline
\end{tabular}


Table A1.g: Croatia: Descriptive Statistics

\begin{tabular}{lccccc}
\hline \hline & Obs. Number & Mean & Std. Dev. & Min & Max \\
\hline Listing (dummy) & 50892 & 0.007 & 0.083 & 0 & 1 \\
ROA (EBIT/TA, \%) & 50892 & 7.628 & 21.304 & -900 & 623.077 \\
ROK(EBIT/FIXED ASSET, \%) & 47315 & 1.223 & 9.625 & -209 & 1040 \\
ROA (P/L before tax, \%) & 50637 & 7.313 & 16.164 & -99.33 & 99.74 \\
ROK & 47135 & 113.847 & 858.463 & -16789.85 & 97952.258 \\
D/A (ratio) & 50892 & 0.581 & 0.265 & 0 & 1 \\
Age & 50675 & 13.514 & 9.44 & 1 & 319 \\
Total Assets & 50892 & 2150.091 & 18732.167 & 1 & 944563 \\
Number of Workers & 50892 & 23.284 & 136.052 & 1 & 6706 \\
\hline
\end{tabular}

Croatia: Correlation Table

\begin{tabular}{|c|c|c|c|c|c|c|c|c|c|}
\hline & 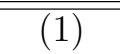 & $\overline{(2)}$ & $\overline{(\overline{(3)}}$ & $(4)$ & 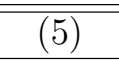 & $\overline{(6)}$ & $\overline{(7)}$ & $\overline{(8)}$ & 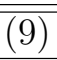 \\
\hline (1) Listing (dummy) & 1 & & & & & & & & \\
\hline (2) $\mathrm{ROA}(\mathrm{EBIT} / \mathrm{TA}, \%)$ & -0.037 & 1 & & & & & & & \\
\hline (3) ROK(EBIT/FIXED ASSET, \%) & -0.011 & 0.266 & 1 & & & & & & \\
\hline (4) ROA (P/L before tax, \%) & -0.044 & 0.925 & 0.263 & 1 & & & & & \\
\hline (5) $\mathrm{ROK}$ & -0.011 & 0.262 & 0.979 & 0.279 & 1 & & & & \\
\hline (6) $\mathrm{D} / \mathrm{A}$ (ratio) & -0.041 & -0.181 & -0.054 & -0.227 & -0.063 & 1 & & & \\
\hline (7) Age & 0.338 & -0.083 & -0.033 & -0.096 & -0.033 & -0.183 & 1 & & \\
\hline (8) Total Assets & 0.437 & -0.016 & -0.01 & -0.025 & -0.011 & -0.004 & 0.283 & 1 & \\
\hline (9) Number of Workers & 0.466 & -0.01 & -0.012 & -0.02 & -0.013 & -0.012 & 0.31 & 0.873 & 1 \\
\hline
\end{tabular}


Table A1.h: Czech Republic: Descriptive Statistics

\begin{tabular}{lccccc}
\hline \hline & Obs. Number & Mean & Std. Dev. & Min & Max \\
\hline Listing (dummy) & 77284 & 0.001 & 0.029 & 0 & 1 \\
ROA (EBIT/TA, \%) & 77284 & 8.656 & 42.674 & -1266.667 & 7500 \\
ROK(EBIT/FIXED ASSET, \%) & 70267 & 1.221 & 8.796 & -130 & 699 \\
ROA (P/L before tax, \%) & 76921 & 7.357 & 16.765 & -98.68 & 100 \\
ROK & 70061 & 108.603 & 789.671 & -17430.6 & 48971.441 \\
D/A (ratio) & 77284 & 0.439 & 4.528 & -1148 & 1 \\
Age & 77284 & 12.657 & 6.983 & 1 & 71 \\
Total Assets & 77284 & 2682.272 & 15402.264 & -26423 & 1231431 \\
Number of Workers & 77284 & 38.719 & 103.848 & 3 & 4500 \\
\hline
\end{tabular}

Czech Republic: Correlation Table

\begin{tabular}{|c|c|c|c|c|c|c|c|c|c|}
\hline Variables & $\overline{(1)}$ & 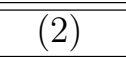 & 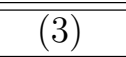 & $\overline{(4)}$ & 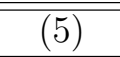 & (6) & 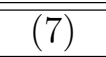 & 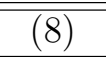 & $\overline{(9)}$ \\
\hline (1) Listing (dummy) & 1 & & & & & & & & \\
\hline (2) ROA (EBIT/TA, \%) & -0.012 & 1 & & & & & & & \\
\hline (3) ROK(EBIT/FIXED ASSET, \%) & -0.004 & 0.304 & 1 & & & & & & \\
\hline (4) ROA (P/L before tax, \%) & -0.011 & 0.972 & 0.301 & 1 & & & & & \\
\hline (5) $\mathrm{ROK}$ & -0.004 & 0.3 & 0.984 & 0.309 & 1 & & & & \\
\hline (6) $\mathrm{D} / \mathrm{A}$ (ratio) & -0.006 & -0.176 & -0.061 & -0.211 & -0.067 & 1 & & & \\
\hline (7) Age & 0.036 & -0.14 & -0.057 & -0.127 & -0.052 & -0.243 & 1 & & \\
\hline (8) Total Assets & 0.22 & -0.023 & -0.017 & -0.016 & -0.015 & -0.012 & 0.069 & 1 & \\
\hline (9) Number of Workers & 0.265 & -0.036 & -0.03 & -0.031 & -0.028 & -0.023 & 0.177 & 0.636 & 1 \\
\hline
\end{tabular}


Table A1.i: Denmark: Descriptive Statistics

\begin{tabular}{lccccc}
\hline \hline & Obs. Number & Mean & Std. Dev. & Min & Max \\
\hline Listing (dummy) & 3342 & 0.093 & 0.29 & 0 & 1 \\
ROA (EBIT/TA, \%) & 3342 & 9.171 & 22.924 & -318.914 & 244.828 \\
ROK(EBIT/FIXED ASSET, \%) & 3155 & 1.193 & 14.937 & -471 & 328 \\
ROA (P/L before tax, \%) & 3327 & 8.773 & 18.919 & -96.42 & 98.38 \\
ROK & 3142 & 130.33 & 1277.863 & -28817.045 & 32694.199 \\
D/A (ratio) & 3342 & 0.545 & 0.238 & -0.067 & 0.997 \\
Age & 3342 & 21.688 & 23.224 & 1 & 224 \\
Total Assets & 3342 & 95196.066 & 548972.96 & 5 & 8529015 \\
Number of Workers & 3342 & 313.672 & 1699.901 & 1 & 27350 \\
\hline
\end{tabular}

Denmark: Correlation Table

\begin{tabular}{|c|c|c|c|c|c|c|c|c|c|}
\hline & 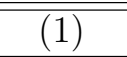 & (2) & 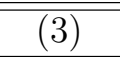 & 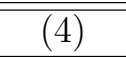 & 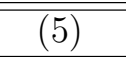 & 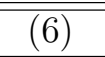 & 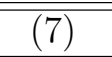 & $\overline{(8)}$ & 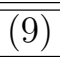 \\
\hline (1) Listing (dummy) & 1 & & & & & & & & \\
\hline (2) ROA (EBIT/TA, \%) & -0.115 & 1 & & & & & & & \\
\hline (3) ROK(EBIT/FIXED ASSET, \%) & -0.087 & 0.282 & 1 & & & & & & \\
\hline (4) ROA (P/L before tax, \%) & -0.122 & 0.934 & 0.295 & 1 & & & & & \\
\hline (5) $\mathrm{ROK}$ & -0.089 & 0.271 & 0.99 & 0.292 & 1 & & & & \\
\hline (6) $\mathrm{D} / \mathrm{A}$ (ratio) & -0.104 & -0.113 & -0.046 & -0.182 & -0.054 & 1 & & & \\
\hline (7) Age & 0.475 & -0.058 & -0.064 & -0.05 & -0.066 & -0.12 & 1 & & \\
\hline (8) Total Assets & 0.351 & -0.009 & -0.02 & -0.017 & -0.018 & 0.024 & 0.224 & 1 & \\
\hline (9) Number of Workers & 0.401 & 0.015 & -0.02 & 0.012 & -0.018 & 0.011 & 0.223 & 0.909 & 1 \\
\hline
\end{tabular}


Table A1.j: Finland: Descriptive Statistics

\begin{tabular}{lccccc}
\hline \hline & Obs. Number & Mean & Std. Dev. & Min & Max \\
\hline Listing (dummy) & 33193 & 0.015 & 0.12 & 0 & 1 \\
ROA (EBIT/TA, \%) & 33193 & 8.538 & 24.568 & -1600 & 988.889 \\
ROK(EBIT/FIXED ASSET, \%) & 32063 & 0.989 & 6.915 & -227 & 283 \\
ROA (P/L before tax, \%) & 33069 & 8.016 & 17.861 & -100 & 100 \\
ROK & 31990 & 97.569 & 692.157 & -9770.58 & 28769.398 \\
D/A (ratio) & 33193 & 0.493 & 0.282 & -1 & 0.999 \\
Age & 33193 & 20.29 & 15.947 & 1 & 183 \\
Total Assets & 33193 & 46063.786 & 887704.811 & 1 & 55086242 \\
Number of Workers & 33193 & 130.349 & 2081.013 & 1 & 132427 \\
\hline
\end{tabular}

Finland: Correlation Table

\begin{tabular}{|c|c|c|c|c|c|c|c|c|c|}
\hline & 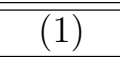 & (2) & (3) & $\overline{(4)}$ & $\overline{(5)}$ & (6) & 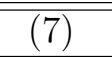 & (8) & (9) \\
\hline (1) Listing (dummy) & 1 & & & & & & & & \\
\hline (2) ROA (EBIT/TA, \%) & -0.028 & 1 & & & & & & & \\
\hline (3) ROK(EBIT/FIXED ASSET, \%) & -0.016 & 0.271 & 1 & & & & & & \\
\hline (4) ROA (P/L before tax, \%) & -0.028 & 0.985 & 0.274 & 1 & & & & & \\
\hline (5) $\mathrm{ROK}$ & -0.016 & 0.263 & 0.988 & 0.271 & 1 & & & & \\
\hline (6) $\mathrm{D} / \mathrm{A}$ (ratio) & 0.027 & -0.21 & -0.087 & -0.272 & -0.096 & 1 & & & \\
\hline (7) Age & 0.303 & -0.089 & -0.046 & -0.076 & -0.043 & -0.121 & 1 & & \\
\hline (8) Total Assets & 0.392 & -0.012 & -0.007 & -0.011 & -0.006 & 0.016 & 0.169 & 1 & \\
\hline (9) Number of Workers & 0.422 & -0.009 & -0.007 & -0.007 & -0.007 & 0.021 & 0.174 & 0.92 & 1 \\
\hline
\end{tabular}


Table A1.k: France: Descriptive Statistics

\begin{tabular}{lccccc}
\hline \hline & Obs. Number & Mean & Std. Dev. & Min & Max \\
\hline Listing (dummy) & 107482 & 0.011 & 0.102 & 0 & 1 \\
ROA (EBIT/TA, \%) & 107482 & 7.179 & 22.035 & -818.069 & 5491.256 \\
ROK(EBIT/FIXED ASSET, \%) & 106523 & 1.135 & 43.121 & -258.889 & 7886 \\
ROA (P/L before tax, \%) & 107392 & 6.558 & 13.544 & -99.96 & 99.93 \\
ROK & 106440 & 78.054 & 594.753 & -75438.211 & 37508.418 \\
D/A (ratio) & 107482 & 0.563 & 0.225 & -3.444 & 1 \\
Age & 107482 & 18.304 & 16.009 & 1 & 352 \\
Total Assets & 107482 & 71258.332 & 1998977.319 & 1 & 132483238 \\
Number of Workers & 107482 & 168.137 & 4079.177 & 1 & 324000 \\
\hline
\end{tabular}

France: Correlation Table

\begin{tabular}{|c|c|c|c|c|c|c|c|c|c|}
\hline & (1) & (2) & 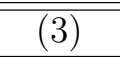 & 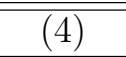 & 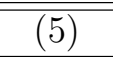 & (6) & 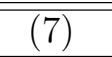 & (8) & (9) \\
\hline (1) Listing (dummy) & 1 & & & & & & & & \\
\hline (2) ROA (EBIT/TA, \%) & -0.074 & 1 & & & & & & & \\
\hline (3) ROK(EBIT/FIXED ASSET, \%) & -0.005 & 0.044 & 1 & & & & & & \\
\hline (4) ROA (P/L before tax, \%) & -0.083 & 0.982 & 0.034 & 1 & & & & & \\
\hline (5) $\mathrm{ROK}$ & -0.035 & 0.272 & 0.097 & 0.283 & 1 & & & & \\
\hline (6) $\mathrm{D} / \mathrm{A}$ (ratio) & -0.019 & -0.141 & 0.007 & -0.195 & -0.073 & 1 & & & \\
\hline (7) Age & 0.197 & -0.113 & -0.002 & -0.09 & -0.012 & -0.277 & 1 & & \\
\hline (8) Total Assets & 0.334 & -0.004 & -0.001 & -0.004 & -0.004 & 0.006 & 0.169 & 1 & \\
\hline (9) Number of Workers & 0.368 & -0.003 & 0 & -0.005 & -0.005 & 0.01 & 0.236 & 0.87 & 1 \\
\hline
\end{tabular}


Table A1.l: Greece: Descriptive Statistics

\begin{tabular}{lccccc}
\hline \hline & Obs. Number & Mean & Std. Dev. & Min & Max \\
\hline Listing (dummy) & 27761 & 0.022 & 0.146 & 0 & 1 \\
ROA (EBIT/TA, \%) & 27761 & 3.886 & 11.321 & -602.381 & 210.096 \\
ROK(EBIT/FIXED ASSET, \%) & 27631 & 0.664 & 4.952 & -179.5 & 219.5 \\
ROA (P/L before tax, \%) & 27747 & 2.375 & 9.612 & -93.52 & 82.45 \\
ROK & 27623 & 51.155 & 488.239 & -19281.35 & 21935.102 \\
D/A (ratio) & 27761 & 0.515 & 0.248 & 0 & 0.999 \\
Age & 27412 & 23.873 & 16.554 & 1 & 156 \\
Total Assets & 27761 & 14653.368 & 104531.848 & 1 & 4445213 \\
Number of Workers & 27761 & 48.011 & 246.234 & 1 & 9314 \\
\hline
\end{tabular}

Greece: Correlation Table

\begin{tabular}{|c|c|c|c|c|c|c|c|c|c|}
\hline Variables & 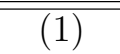 & $\overline{(2)}$ & $\overline{(3)}$ & $\overline{(4)}$ & $\overline{(5)}$ & 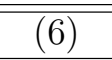 & $\overline{(7)}$ & $\overline{(8)}$ & 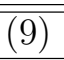 \\
\hline (1) Listing (dummy) & 1 & & & & & & & & \\
\hline (2) ROA (EBIT/TA, \%) & -0.029 & 1 & & & & & & & \\
\hline (3) ROK(EBIT/FIXED ASSET, \%) & -0.018 & 0.322 & 1 & & & & & & \\
\hline (4) ROA (P/L before tax, \%) & -0.049 & 0.979 & 0.32 & 1 & & & & & \\
\hline (5) $\mathrm{ROK}$ & -0.015 & 0.316 & 0.938 & 0.323 & 1 & & & & \\
\hline (6) $\mathrm{D} / \mathrm{A}$ (ratio) & 0.053 & 0.016 & 0.029 & -0.08 & 0.007 & 1 & & & \\
\hline (7) Age & 0.122 & -0.042 & -0.054 & -0.046 & -0.048 & -0.106 & 1 & & \\
\hline (8) Total Assets & 0.382 & 0.012 & -0.013 & 0.003 & -0.01 & 0.039 & 0.133 & 1 & \\
\hline (9) Number of Workers & 0.406 & 0.026 & -0.015 & 0.015 & -0.012 & 0.04 & 0.109 & 0.721 & 1 \\
\hline
\end{tabular}


Table A1.m: Germany: Descriptive Statistics

\begin{tabular}{lccccc}
\hline \hline & Obs. Number & Mean & Std. Dev. & Min & Max \\
\hline Listing (dummy) & 50397 & 0.027 & 0.163 & 0 & 1 \\
ROA (EBIT/TA, \%) & 45812 & 10.603 & 45.305 & -177.466 & 7013.468 \\
ROK(EBIT/FIXED ASSET, \%) & 45349 & 2.312 & 43.847 & -338 & 4659.667 \\
ROA (P/L before tax, \%) & 50346 & 9.176 & 12.833 & -97.92 & 99.46 \\
ROK & 49889 & 206.282 & 4038.987 & -39853.441 & 441459.75 \\
D/A (ratio) & 50397 & 0.61 & 0.239 & 0 & 1 \\
Age & 50368 & 41.794 & 42.844 & 1 & 733 \\
Total Assets & 50397 & 246570.549 & 5081694.763 & 4 & 306546968 \\
Number of Workers & 50397 & 594.868 & 8597.175 & 1 & 427000 \\
\hline
\end{tabular}

Germany: Correlation Table

\begin{tabular}{|c|c|c|c|c|c|c|c|c|c|}
\hline & (1) & $\overline{(2)}$ & 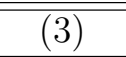 & $\overline{(4)}$ & $\overline{(5)}$ & $\overline{(26)}$ & 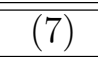 & $\overline{(8)}$ & $\overline{(9)}$ \\
\hline (1) Listing (dummy) & 1 & & & & & & & & \\
\hline (2) ROA (EBIT/TA, \%) & -0.092 & 1 & & & & & & & \\
\hline (3) ROK(EBIT/FIXED ASSET, \%) & -0.009 & 0.068 & 1 & & & & & & \\
\hline (4) ROA (P/L before tax, \%) & -0.086 & 0.983 & 0.069 & 1 & & & & & \\
\hline (5) $\mathrm{ROK}$ & -0.009 & 0.069 & 0.998 & 0.071 & 1 & & & & \\
\hline (6) $\mathrm{D} / \mathrm{A}$ (ratio) & -0.075 & -0.118 & -0.002 & -0.187 & -0.006 & 1 & & & \\
\hline (7) Age & 0.104 & -0.047 & -0.012 & -0.042 & -0.012 & 0.003 & 1 & & \\
\hline (8) Total Assets & 0.256 & -0.016 & -0.002 & -0.013 & -0.002 & 0.011 & 0.05 & 1 & \\
\hline (9) Number of Workers & 0.289 & -0.018 & -0.003 & -0.016 & -0.003 & 0.012 & 0.069 & 0.836 & 1 \\
\hline
\end{tabular}


Table A1.n: Hungary: Descriptive Statistics

\begin{tabular}{lccccc}
\hline \hline & Obs. Number & Mean & Std. Dev. & Min & Max \\
\hline Listing (dummy) & 123852 & 0.001 & 0.024 & 0 & 1 \\
ROA (EBIT/TA, \%) & 123814 & 8.455 & 35.275 & -2562.963 & 4878.571 \\
ROK(EBIT/FIXED ASSET, \%) & 114087 & 0.918 & 7.567 & -247 & 928.667 \\
ROA (P/L before tax, \%) & 122833 & 8.188 & 19.168 & -99.77 & 100 \\
ROK & 113481 & 89.315 & 676.227 & -12511.711 & 85663.266 \\
D/A (ratio) & 123852 & 0.453 & 0.34 & -66.357 & 1 \\
Age & 123849 & 12.88 & 7.919 & 1 & 117 \\
Total Assets & 123852 & 2071.954 & 27302.84 & -18 & 3311281 \\
Number of Workers & 123852 & 26.392 & 138.942 & 1 & 11820 \\
\hline
\end{tabular}

Hungary: Correlation Table

\begin{tabular}{|c|c|c|c|c|c|c|c|c|c|}
\hline & 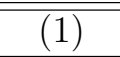 & (2) &  & $\overline{(4)}$ & 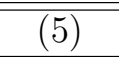 & 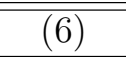 & 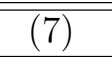 & $\overline{(8)}$ & (9) \\
\hline (1) Listing (dummy) & 1 & & & & & & & & \\
\hline (2) ROA (EBIT/TA, \%) & -0.002 & 1 & & & & & & & \\
\hline (3) ROK(EBIT/FIXED ASSET, \%) & -0.003 & 0.28 & 1 & & & & & & \\
\hline (4) ROA (P/L before tax, \%) & -0.002 & 0.983 & 0.281 & 1 & & & & & \\
\hline (5) $\mathrm{ROK}$ & -0.003 & 0.281 & 0.988 & 0.288 & 1 & & & & \\
\hline (6) $\mathrm{D} / \mathrm{A}$ (ratio) & -0.002 & -0.102 & -0.006 & -0.139 & -0.013 & 1 & & & \\
\hline (7) Age & 0.095 & -0.122 & -0.061 & -0.116 & -0.06 & -0.188 & 1 & & \\
\hline (8) Total Assets & 0.372 & -0.008 & -0.006 & -0.008 & -0.006 & 0.007 & 0.139 & 1 & \\
\hline (9) Number of Workers & 0.399 & -0.014 & -0.01 & -0.015 & -0.01 & 0.026 & 0.184 & 0.743 & 1 \\
\hline
\end{tabular}


Table A1.o: India: Descriptive Statistics

\begin{tabular}{lccccc}
\hline \hline & Obs. Number & Mean & Std. Dev. & Min & Max \\
\hline Listing (dummy) & 3079 & 0.974 & 0.158 & 0 & 1 \\
ROA (EBIT/TA, \%) & 3079 & 7.411 & 9.936 & -133.333 & 168.078 \\
ROK(EBIT/FIXED ASSET, \%) & 3070 & 0.028 & 11.5 & -627.741 & 78.5 \\
ROA (P/L before tax, \%) & 3075 & 5.671 & 10.234 & -85.8 & 76.98 \\
ROK & 3066 & 0.112 & 945.913 & -49299.746 & 8357.625 \\
D/A (ratio) & 3079 & 0.548 & 0.228 & 0 & 0.999 \\
Age & 3079 & 36.546 & 22.126 & 1 & 141 \\
Total Assets & 3079 & 934477.406 & 5089997.387 & 7 & 109009446 \\
Number of Workers & 3079 & 2612.867 & 7613.794 & 1 & 86548 \\
\hline
\end{tabular}

India: Correlation Table

\begin{tabular}{|c|c|c|c|c|c|c|c|c|c|}
\hline & $\overline{(1)}$ & $\overline{(2)}$ & $\overline{(3)}$ & $\overline{(4)}$ & $(5)$ & (6) & $\overline{(7)}$ & $\overline{(8)}$ & $\overline{(9)}$ \\
\hline (1) Listing (dummy) & 1 & & & & & & & & \\
\hline (2) ROA (EBIT/TA, \%) & 0.02 & 1 & & & & & & & \\
\hline (3) ROK(EBIT/FIXED ASSET, \%) & -0.005 & 0.146 & 1 & & & & & & \\
\hline (4) ROA (P/L before tax, \%) & 0.006 & 0.814 & 0.108 & 1 & & & & & \\
\hline (5) $\mathrm{ROK}$ & -0.006 & 0.15 & 0.966 & 0.158 & 1 & & & & \\
\hline (6) $\mathrm{D} / \mathrm{A}$ (ratio) & -0.018 & -0.099 & -0.021 & -0.337 & -0.039 & 1 & & & \\
\hline (7) Age & -0.012 & 0.031 & -0.015 & 0.074 & -0.007 & -0.012 & 1 & & \\
\hline (8) Total Assets & 0.025 & 0.005 & 0.002 & 0.006 & 0.002 & 0.074 & 0.133 & 1 & \\
\hline (9) Number of Workers & 0.037 & 0.079 & 0.004 & 0.06 & 0.005 & 0.099 & 0.253 & 0.614 & 1 \\
\hline
\end{tabular}


Table A1.p: Iceland: Descriptive Statistics

\begin{tabular}{lccccc}
\hline \hline & Obs. Number & Mean & Std. Dev. & Min & Max \\
\hline Listing (dummy) & 2549 & 0.024 & 0.153 & 0 & 1 \\
ROA (EBIT/TA, \%) & 2549 & 14.962 & 31.887 & -210 & 940 \\
ROK(EBIT/FIXED ASSET, \%) & 2367 & 1.379 & 6.671 & -49.5 & 149 \\
ROA (P/L before tax, \%) & 2527 & 12.556 & 21.975 & -86.14 & 96.58 \\
ROK & 2351 & 125.772 & 656.833 & -6605.39 & 13965.12 \\
D/A (ratio) & 2549 & 0.531 & 0.268 & 0 & 0.998 \\
Age & 2549 & 17.433 & 13.96 & 1 & 109 \\
Total Assets & 2549 & 10595.965 & 83329.068 & 1 & 1727711 \\
Number of Workers & 2549 & 35.332 & 256.455 & 1 & 4912 \\
\hline
\end{tabular}

Iceland: Correlation Table

\begin{tabular}{|c|c|c|c|c|c|c|c|c|c|}
\hline & 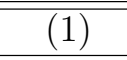 & 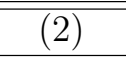 & (3) & $(\overline{(4)}$ & 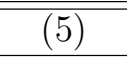 & 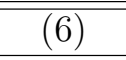 & 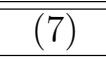 & 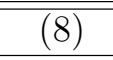 & 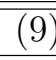 \\
\hline (1) Listing (dummy) & 1 & & & & & & & & \\
\hline (2) ROA (EBIT/TA, \%) & -0.04 & 1 & & & & & & & \\
\hline (3) ROK(EBIT/FIXED ASSET, \%) & -0.031 & 0.371 & 1 & & & & & & \\
\hline (4) ROA (P/L before tax, \%) & -0.034 & 0.944 & 0.36 & 1 & & & & & \\
\hline (5) $\mathrm{ROK}$ & -0.029 & 0.362 & 0.97 & 0.395 & 1 & & & & \\
\hline (6) $\mathrm{D} / \mathrm{A}$ (ratio) & 0.042 & -0.134 & -0.082 & -0.219 & -0.108 & 1 & & & \\
\hline (7) Age & 0.423 & -0.09 & -0.042 & -0.067 & -0.039 & -0.038 & 1 & & \\
\hline (8) Total Assets & 0.585 & -0.038 & -0.024 & -0.037 & -0.023 & 0.022 & 0.153 & 1 & \\
\hline (9) Number of Workers & 0.537 & -0.037 & -0.023 & -0.038 & -0.022 & 0.026 & 0.157 & 0.965 & 1 \\
\hline
\end{tabular}


Table A1.q: Ireland: Descriptive Statistics

\begin{tabular}{lccccc}
\hline \hline & Obs. Number & Mean & Std. Dev. & Min & Max \\
\hline Listing (dummy) & 2545 & 0.102 & 0.303 & 0 & 1 \\
ROA (EBIT/TA, \%) & 2545 & 3.254 & 19.122 & -363.38 & 100 \\
ROK(EBIT/FIXED ASSET, \%) & 2456 & 1.345 & 18.823 & -81 & 564 \\
ROA (P/L before tax, \%) & 1950 & 4.131 & 15.004 & -83.44 & 95.87 \\
ROK & 1886 & 172.22 & 2116.822 & -7330.4 & 55946.516 \\
D/A (ratio) & 2545 & 0.465 & 0.271 & 0 & 0.998 \\
Age & 2545 & 23.809 & 20.208 & 1 & 177 \\
Total Assets & 2545 & 1037657.31 & 6929072.976 & 2 & 135840700 \\
Number of Workers & 2545 & 2113.597 & 11442.182 & 1 & 209000 \\
\hline
\end{tabular}

Ireland: Correlation Table

\begin{tabular}{|c|c|c|c|c|c|c|c|c|c|}
\hline & 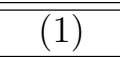 & (2) & 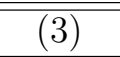 & 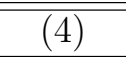 & 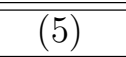 & 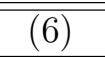 & 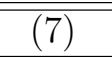 & $\overline{(8)}$ & $\overline{(9)}$ \\
\hline (1) Listing (dummy) & 1 & & & & & & & & \\
\hline (2) $\mathrm{ROA}(\mathrm{EBIT} / \mathrm{TA}, \%)$ & -0.061 & 1 & & & & & & & \\
\hline (3) ROK(EBIT/FIXED ASSET, \%) & -0.035 & 0.134 & 1 & & & & & & \\
\hline (4) ROA (P/L before tax) & -0.085 & 0.945 & 0.131 & 1 & & & & & \\
\hline (5) $\mathrm{ROK}$ & -0.035 & 0.134 & 0.999 & 0.136 & 1 & & & & \\
\hline (6) $\mathrm{D} / \mathrm{A}$ (ratio) & 0.052 & 0.035 & 0.002 & 0.001 & -0.002 & 1 & & & \\
\hline (7) Age & 0.276 & -0.031 & -0.039 & -0.025 & -0.04 & 0.001 & 1 & & \\
\hline (8) Total Assets & 0.431 & 0.005 & -0.013 & -0.009 & -0.014 & 0.056 & 0.24 & 1 & \\
\hline (9) Number of Workers & 0.511 & 0.044 & -0.016 & 0.029 & -0.016 & 0.106 & 0.276 & 0.683 & 1 \\
\hline
\end{tabular}


Table A1.r: Italy: Descriptive Statistics

\begin{tabular}{lccccc}
\hline \hline & Obs. Number & Mean & Std. Dev. & Min & Max \\
\hline Listing (dummy) & 546402 & 0.001 & 0.027 & 0 & 1 \\
ROA (EBIT/TA, \%) & 546402 & 5.388 & 32.092 & -19261.549 & 8259.259 \\
ROK(EBIT/FIXED ASSET, \%) & 542873 & 0.904 & 7.555 & -961.5 & 1220 \\
ROA (P/L before tax, \%) & 546226 & 4.259 & 9.793 & -99.97 & 99.13 \\
ROK & 542709 & 77.444 & 721.659 & -39582.75 & 121701.18 \\
D/A (ratio) & 546402 & 0.717 & 0.218 & -3.636 & 1 \\
Age & 546401 & 18.846 & 14.378 & 1 & 144 \\
Total Assets & 546402 & 7095.184 & 172914.108 & 1 & 43916703 \\
Number of Workers & 546402 & 21.636 & 301.277 & 1 & 75197 \\
\hline
\end{tabular}

Italy: Correlation Table

\begin{tabular}{|c|c|c|c|c|c|c|c|c|c|}
\hline & (1) & 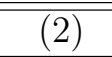 & (3) & $\overline{(4)}$ & $\overline{(5)}$ & 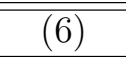 & 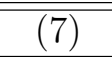 & $\overline{(8)}$ & 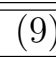 \\
\hline (1) Listing (dummy) & 1 & & & & & & & & \\
\hline (2) ROA (EBIT/TA, \%) & -0.002 & 1 & & & & & & & \\
\hline (3) ROK(EBIT/FIXED ASSET, \%) & -0.004 & 0.249 & 1 & & & & & & \\
\hline (4) $\mathrm{ROA}(\mathrm{P} / \mathrm{L}$ before tax, \%) & -0.008 & 0.336 & 0.243 & 1 & & & & & \\
\hline (5) $\mathrm{ROK}$ & -0.004 & 0.085 & 0.972 & 0.255 & 1 & & & & \\
\hline (6) $\mathrm{D} / \mathrm{A}$ (ratio) & -0.014 & -0.071 & -0.047 & -0.264 & -0.061 & 1 & & & \\
\hline (7) Age & 0.054 & -0.033 & -0.043 & -0.087 & -0.04 & -0.274 & 1 & & \\
\hline (8) Total Assets & 0.319 & -0.002 & -0.003 & -0.006 & -0.003 & -0.008 & 0.052 & 1 & \\
\hline (9) Number of Workers & 0.336 & -0.002 & -0.004 & -0.005 & -0.004 & -0.007 & 0.065 & 0.964 & 1 \\
\hline
\end{tabular}


Table A1.s: Japan: Descriptive Statistics

\begin{tabular}{lccccc}
\hline \hline & Obs. Number & Mean & Std. Dev. & Min & Max \\
\hline Listing (dummy) & 182306 & 0.069 & 0.254 & 0 & 1 \\
ROA (EBIT/TA, \%) & 182306 & 2.744 & 9.224 & -871.429 & 206.579 \\
ROK(EBIT/FIXED ASSET, \%) & 181834 & 0.233 & 3.353 & -129 & 468.667 \\
ROA (P/L before tax, \%) & 182217 & 3.414 & 8.113 & -99.850 & 97.100 \\
ROK & 181751 & 27.639 & 337.928 & -10357.950 & 50840.047 \\
D/A (ratio) & 182306 & 0.641 & 0.248 & 0 & 1 \\
Age & 182306 & 37.986 & 20.155 & 1 & 184 \\
Total Assets & 182306 & 197752.211 & 3640856.891 & 2 & 435075307 \\
Number of Workers & 182306 & 472.151 & 6196.925 & 1 & 384586 \\
\hline
\end{tabular}

Japan: Correlation Table

\begin{tabular}{|c|c|c|c|c|c|c|c|c|c|}
\hline & 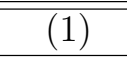 & $\overline{(2)}$ & $\overline{(3)}$ & $\overline{(4)}$ & $\overline{(5)}$ & $(6)$ & $(7)$ & $(8)$ & $\overline{(9)}$ \\
\hline (1) Listing (dummy) & 1 & & & & & & & & \\
\hline (2) ROA (EBIT/TA, \%) & 0.038 & 1 & & & & & & & \\
\hline (3) ROK(EBIT/FIXED ASSET, \%) & -0.013 & 0.244 & 1 & & & & & & \\
\hline (4) ROA (P/L before tax, \%) & 0.015 & 0.892 & 0.228 & 1 & & & & & \\
\hline (5) $\mathrm{ROK}$ & -0.016 & 0.22 & 0.975 & 0.238 & 1 & & & & \\
\hline (6) $\mathrm{D} / \mathrm{A}$ (ratio) & -0.197 & -0.079 & -0.006 & -0.139 & -0.014 & 1 & & & \\
\hline (7) Age & 0.304 & -0.034 & -0.057 & -0.053 & -0.064 & -0.270 & 1 & & \\
\hline (8) Total Assets & 0.182 & 0.01 & -0.002 & 0.005 & -0.003 & -0.018 & 0.092 & 1 & \\
\hline (9) Number of Workers & 0.250 & 0.016 & -0.003 & 0.007 & -0.004 & -0.029 & 0.133 & 0.790 & 1 \\
\hline
\end{tabular}


Table A1.t: Korea: Descriptive Statistics

\begin{tabular}{lccccc}
\hline \hline & Obs. Number & Mean & Std. Dev. & Min & Max \\
\hline Listing (dummy) & 168946 & 0.018 & 0.132 & 0 & 1 \\
ROA (EBIT/TA, \%) & 168934 & 7.837 & 28.998 & -6200 & 3800 \\
ROK(EBIT/FIXED ASSET, \%) & 168568 & 0.441 & 2.483 & -167 & 222.087 \\
ROA (P/L before tax, \%) & 168608 & 7.047 & 11.38 & -99.74 & 100 \\
ROK & 168268 & 43.871 & 285.538 & -35169.598 & 51693.449 \\
D/A (ratio) & 168946 & 0.576 & 0.222 & -7.722 & 1 \\
Age & 168245 & 11.813 & 8.701 & 1 & 119 \\
Total Assets & 168946 & 10340.757 & 54616.658 & 1 & 6237490 \\
Number of Workers & 168946 & 34.701 & 75.001 & 1 & 6002 \\
\hline
\end{tabular}

Korea: Correlation Table

\begin{tabular}{|c|c|c|c|c|c|c|c|c|c|}
\hline & 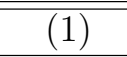 & $\overline{(2)}$ & $\overline{(3)}$ & $\overline{(4)}$ & $\overline{(5)}$ & $(6)$ & $(7)$ & $(8)$ & $\overline{(9)}$ \\
\hline (1) Listing (dummy) & 1 & & & & & & & & \\
\hline (2) ROA (EBIT/TA, \%) & -0.048 & 1 & & & & & & & \\
\hline (3) ROK(EBIT/FIXED ASSET, \%) & -0.019 & 0.354 & 1 & & & & & & \\
\hline (4) $\mathrm{ROA}(\mathrm{P} / \mathrm{L}$ befor ) & -0.065 & 0.843 & 0.321 & 1 & & & & & \\
\hline (5) $\mathrm{ROK}$ & -0.018 & 0.239 & 0.762 & 0.292 & 1 & & & & \\
\hline (6) $\mathrm{D} / \mathrm{A}$ (ratio) & -0.075 & -0.192 & -0.084 & -0.305 & -0.091 & 1 & & & \\
\hline (7) Age & 0.174 & -0.092 & -0.076 & -0.098 & -0.062 & -0.206 & 1 & & \\
\hline (8) Total Assets & 0.231 & -0.032 & -0.02 & -0.033 & -0.017 & -0.021 & 0.16 & 1 & \\
\hline (9) Number of Workers & 0.287 & -0.054 & -0.04 & -0.059 & -0.034 & -0.015 & 0.289 & 0.712 & 1 \\
\hline
\end{tabular}


Table A1.u: Latvia: Descriptive Statistics

\begin{tabular}{lccccc}
\hline \hline & Obs. Number & Mean & Std. Dev. & Min & Max \\
\hline Listing (dummy) & 21501 & 0.006 & 0.078 & 0 & 1 \\
ROA (EBIT/TA, \%) & 21501 & 10.107 & 36.147 & -700 & 1028.571 \\
ROK(EBIT/FIXED ASSET, \%) & 18558 & 1.107 & 7.217 & -167 & 339 \\
ROA (P/L before tax, \%) & 21096 & 9.229 & 24.326 & -98.47 & 99.99 \\
ROK & 18355 & 100.942 & 672.693 & -22227.549 & 33845.758 \\
D/A (ratio) & 21501 & 0.546 & 0.29 & -1 & 1 \\
Age & 21501 & 10.541 & 8.677 & 1 & 150 \\
Total Assets & 21501 & 1447.181 & 11686.931 & 1 & 494248 \\
Number of Workers & 21501 & 21.953 & 62.354 & 1 & 1679 \\
\hline
\end{tabular}

Latvia: Correlation Table

\begin{tabular}{|c|c|c|c|c|c|c|c|c|c|}
\hline & (1) & (2) & $\overline{(3)}$ & (4) & $\overline{(5)}$ & $\overline{(6)}$ & (7) & $(8)$ & $\overline{(9)}$ \\
\hline (1) Listing (dummy) & 1 & & & & & & & & \\
\hline (2) ROA (EBIT/TA, \%) & -0.03 & 1 & & & & & & & \\
\hline (3) ROK(EBIT/FIXED ASSET, \%) & -0.013 & 0.32 & 1 & & & & & & \\
\hline (4) $\mathrm{ROA}(\mathrm{P} / \mathrm{L}$ befor $)$ & -0.032 & 0.956 & 0.32 & 1 & & & & & \\
\hline (5) $\mathrm{ROK}$ & -0.012 & 0.298 & 0.982 & 0.317 & 1 & & & & \\
\hline (6) $\mathrm{D} / \mathrm{A}$ (ratio) & -0.053 & -0.194 & -0.073 & -0.219 & -0.076 & 1 & & & \\
\hline (7) Age & 0.464 & -0.098 & -0.041 & -0.098 & -0.036 & -0.18 & 1 & & \\
\hline (8) Total Assets & 0.243 & -0.016 & -0.011 & -0.02 & -0.011 & -0.007 & 0.156 & 1 & \\
\hline (9) Number of Workers & 0.407 & -0.015 & -0.024 & -0.021 & -0.025 & -0.02 & 0.382 & 0.599 & 1 \\
\hline
\end{tabular}

Table A1.v: Malaysia: Descriptive Statistics

\begin{tabular}{lccccc}
\hline \hline & Obs. Number & Mean & Std. Dev. & Min & Max \\
\hline Listing (dummy) & 594 & 0.157 & 0.364 & 0 & 1 \\
ROA (EBIT/TA, \%) & 594 & 7.052 & 12.883 & -50.353 & 102.585 \\
ROK(EBIT/FIXED ASSET, \%) & 594 & 0.862 & 5.968 & -3.8 & 132 \\
ROA (P/L before tax, \%) & 592 & 6.338 & 12.001 & -55.36 & 94.92 \\
ROK & 592 & 79.239 & 484.79 & -361.027 & 10100.46 \\
D/A (ratio) & 594 & 0.518 & 0.23 & 0.027 & 0.998 \\
Age & 594 & 22.606 & 13.051 & 1 & 67 \\
Total Assets & 594 & 98592.15 & 239639.832 & 79 & 2529464 \\
Number of Workers & 594 & 700.463 & 1747.562 & 1 & 12000 \\
\hline
\end{tabular}


Malaysia: Correlation Table

\begin{tabular}{|c|c|c|c|c|c|c|c|c|c|}
\hline & $(1)$ & $(2)$ & $(3)$ & $(4)$ & $(5)$ & $(6)$ & (7) & $(8)$ & $(9)$ \\
\hline (1) Listing (dummy) & 1 & & & & & & & & \\
\hline (2) ROA (EBIT/TA, \%) & 0.089 & 1 & & & & & & & \\
\hline (3) ROK(EBIT/FIXED ASSET, \%) & -0.046 & 0.296 & 1 & & & & & & \\
\hline (4) ROA (P/L before tax, \%) & 0.079 & 0.968 & 0.245 & 1 & & & & & \\
\hline (5) $\mathrm{ROK}$ & -0.053 & 0.309 & 0.991 & 0.27 & 1 & & & & \\
\hline (6) $\mathrm{D} / \mathrm{A}$ (ratio) & -0.197 & -0.077 & -0.014 & -0.153 & -0.026 & 1 & & & \\
\hline (7) Age & -0.007 & 0.157 & -0.115 & 0.195 & -0.122 & -0.198 & 1 & & \\
\hline (8) Total Assets & 0.481 & 0.09 & -0.035 & 0.107 & -0.037 & -0.162 & 0.105 & 1 & \\
\hline (9) Number of Workers & 0.517 & 0.064 & -0.04 & 0.074 & -0.044 & -0.184 & -0.011 & 0.66 & 1 \\
\hline
\end{tabular}

Table A1.w: Netherlands: Descriptive Statistics

\begin{tabular}{lccccc}
\hline \hline & Obs. Number & Mean & Std. Dev. & Min & Max \\
\hline Listing (dummy) & 2005 & 0.17 & 0.376 & 0 & 1 \\
ROA (EBIT/TA, \%) & 1999 & 8.726 & 30.691 & -518.455 & 325 \\
ROK(EBIT/FIXED ASSET, \%) & 1929 & 3.268 & 47.513 & -174.5 & 1887 \\
ROA (P/L before tax, \%) & 1947 & 7.701 & 14.77 & -97.82 & 96.09 \\
ROK & 1882 & 311.031 & 4642.671 & -11834.76 & 185728.5 \\
D/A (ratio) & 2005 & 0.495 & 2.194 & -95 & 0.998 \\
Age & 2005 & 38.097 & 35.458 & 1 & 252 \\
Total Assets & 2005 & 2239017.389 & 12194362.89 & -17 & 136644596 \\
Number of Workers & 2005 & 4116.639 & 20531.757 & 1 & 238162 \\
\hline
\end{tabular}

Netherlands: Correlation Table

\begin{tabular}{|c|c|c|c|c|c|c|c|c|c|}
\hline & $(1)$ & $(2)$ & $(3)$ & $(4)$ & $(5)$ & $(6)$ & $(7)$ & $(8)$ & $(9)$ \\
\hline (1) Listing (dummy) & 1 & & & & & & & & \\
\hline (2) ROA (EBIT/TA, \%) & -0.076 & 1 & & & & & & & \\
\hline (3) ROK(EBIT/FIXED ASSET, \%) & -0.031 & 0.13 & 1 & & & & & & \\
\hline (4) ROA (P/L before tax, \%) & -0.094 & 0.784 & 0.096 & 1 & & & & & \\
\hline (5) $\mathrm{ROK}$ & -0.03 & 0.087 & 0.985 & 0.102 & 1 & & & & \\
\hline (6) $\mathrm{D} / \mathrm{A}$ (ratio) & 0.022 & -0.149 & 0.009 & -0.199 & 0.007 & 1 & & & \\
\hline (7) Age & 0.17 & -0.058 & -0.012 & -0.069 & -0.013 & 0.005 & 1 & & \\
\hline (8) Total Assets & 0.381 & -0.045 & -0.013 & -0.056 & -0.012 & 0.167 & 0.089 & 1 & \\
\hline (9) Number of Workers & 0.406 & -0.054 & -0.014 & -0.068 & -0.013 & 0.146 & 0.16 & 0.932 & 1 \\
\hline
\end{tabular}


Table A1.x: Norway: Descriptive Statistics

\begin{tabular}{lccccc}
\hline \hline & Obs. Number & Mean & Std. Dev. & Min & Max \\
\hline Listing (dummy) & 6412 & 0.04 & 0.197 & 0 & 1 \\
ROA (EBIT/TA, \%) & 6412 & 7.65 & 30.512 & -588.235 & 1000 \\
ROK(EBIT/FIXED ASSET, \%) & 5854 & -0.633 & 81.75 & -3831 & 490.5 \\
ROA (P/L before tax, \%) & 6368 & 7.664 & 20.608 & -100 & 99.71 \\
ROK & 5824 & 65.972 & 5426.704 & -362353.188 & 49896.883 \\
D/A (ratio) & 6412 & 0.531 & 0.287 & -6.543 & 0.998 \\
Age & 6412 & 16.266 & 19.024 & 1 & 362 \\
Total Assets & 6412 & 55947.843 & 778111.718 & -49 & 22129215 \\
Number of Workers & 6412 & 120.168 & 1264.833 & 1 & 32026 \\
\hline
\end{tabular}

Norway: Correlation Table

\begin{tabular}{lccccccccc}
\hline \hline & $(1)$ & $(2)$ & $(3)$ & $(4)$ & $(5)$ & $(6)$ & $(7)$ & $(8)$ & $(9)$ \\
\hline (1) Listing (dummy) & 1 & & & & & & & & \\
(2) ROA (EBIT/TA, \%) & -0.134 & 1 & & & & & & & \\
(3) ROK(EBIT/FIXED ASSET, \%) & -0.115 & 0.124 & 1 & & & & & & \\
(4) ROA (P/L before tax) & -0.135 & 0.962 & 0.118 & 1 & & & & & \\
(5) ROK & -0.113 & 0.123 & 0.996 & 0.123 & 1 & & & & \\
(6) D/A (ratio) & -0.073 & 0.017 & 0.036 & -0.047 & 0.029 & 1 & & & \\
(7) Age & 0.377 & -0.018 & 0.007 & -0.012 & 0.007 & -0.103 & 1 & & \\
(8) Total Assets & 0.344 & -0.013 & -0.001 & -0.017 & -0.001 & -0.02 & 0.579 & 1 & \\
(9) Number of Workers & 0.426 & -0.013 & -0.001 & -0.019 & -0.001 & -0.003 & 0.722 & 0.875 & 1 \\
\hline
\end{tabular}

Table A1.y: Poland: Descriptive Statistics

\begin{tabular}{lccccc}
\hline \hline & Obs. Number & Mean & Std. Dev. & Min & Max \\
\hline Listing (dummy) & 24597 & 0.024 & 0.153 & 0 & 1 \\
ROA (EBIT/TA, \%) & 24570 & 9.816 & 67.827 & -8800 & 800 \\
ROK(EBIT/FIXED ASSET, \%) & 23588 & 1.294 & 19.618 & -387 & 2399 \\
ROA (P/L before tax, \%) & 24420 & 9.223 & 17.21 & -100 & 100 \\
ROK & 23484 & 125.085 & 1915.158 & -28112 & 232948.516 \\
D/A (ratio) & 24597 & 0.463 & 0.373 & -43 & 1 \\
Age & 24597 & 17.08 & 19.076 & 1 & 253 \\
Total Assets & 24597 & 13901.688 & 293227.377 & -16 & 17257016 \\
Number of Workers & 24597 & 114.238 & 471.551 & 1 & 22956 \\
\hline
\end{tabular}


Poland: Correlation Table

\begin{tabular}{|c|c|c|c|c|c|c|c|c|c|}
\hline & $\overline{(1)}$ & (2) & $\overline{(3)}$ & $(4)$ & $\overline{(5)}$ & 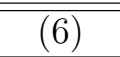 & $\overline{(7)}$ & $(8)$ & (9) \\
\hline (1) Listing (dummy) & 1 & & & & & & & & \\
\hline (2) ROA (EBIT/TA, \%) & -0.065 & 1 & & & & & & & \\
\hline (3) ROK(EBIT/FIXED ASSET, \%) & -0.007 & 0.146 & 1 & & & & & & \\
\hline (4) $\mathrm{ROA}(\mathrm{P} / \mathrm{L}$ befor $)$ & -0.069 & 0.956 & 0.145 & 1 & & & & & \\
\hline (5) $\mathrm{ROK}$ & -0.005 & 0.145 & 0.994 & 0.151 & 1 & & & & \\
\hline (6) $\mathrm{D} / \mathrm{A}$ (ratio) & 0.006 & -0.194 & -0.014 & -0.266 & -0.02 & 1 & & & \\
\hline (7) Age & 0.151 & -0.103 & -0.028 & -0.098 & -0.027 & -0.086 & 1 & & \\
\hline (8) Total Assets & 0.162 & -0.013 & -0.003 & -0.012 & -0.003 & 0.006 & 0.069 & 1 & \\
\hline (9) Number of Workers & 0.282 & -0.025 & -0.011 & -0.027 & -0.011 & 0.003 & 0.177 & 0.827 & 1 \\
\hline
\end{tabular}

Table A1.z: Romania: Descriptive Statistics

\begin{tabular}{lccccc}
\hline \hline & Obs. Number & Mean & Std. Dev. & Min & Max \\
\hline Listing (dummy) & 160172 & 0.006 & 0.08 & 0 & 1 \\
ROA (EBIT/TA, \%) & 160172 & 13.394 & 38.512 & -4337.5 & 6000 \\
ROK(EBIT/FIXED ASSET, \%) & 143468 & 1.22 & 8.275 & -258 & 1231 \\
ROA (P/L before tax, \%) & 158404 & 11.64 & 22.239 & -99.71 & 100 \\
ROK & 142563 & 112.559 & 736.823 & -19038.801 & 124511.398 \\
D/A (ratio) & 160172 & 0.498 & 6.616 & -2628 & 1 \\
Age & 160172 & 11.667 & 6.907 & 1 & 144 \\
Total Assets & 160172 & 1037.558 & 12287.089 & -75 & 2345003 \\
Number of Workers & 160172 & 25.407 & 114.776 & 1 & 33809 \\
\hline
\end{tabular}

Romania: Correlation Table

\begin{tabular}{|c|c|c|c|c|c|c|c|c|c|}
\hline & $(1)$ & $(2)$ & $(3)$ & $(4)$ & $(5)$ & $(6)$ & $(7)$ & $(8)$ & $(9)$ \\
\hline (1) Listing (dummy) & 1 & & & & & & & & \\
\hline (2) ROA (EBIT/TA, \%) & -0.039 & 1 & & & & & & & \\
\hline (3) ROK(EBIT/FIXED ASSET, \%) & -0.012 & 0.29 & 1 & & & & & & \\
\hline (4) ROA (P/L before tax, \%) & -0.04 & 0.982 & 0.291 & 1 & & & & & \\
\hline (5) $\mathrm{ROK}$ & -0.012 & 0.288 & 0.989 & 0.296 & 1 & & & & \\
\hline (6) $\mathrm{D} / \mathrm{A}$ (ratio) & -0.048 & -0.234 & -0.077 & -0.264 & -0.082 & 1 & & & \\
\hline (7) Age & 0.165 & -0.148 & -0.075 & -0.148 & -0.074 & -0.166 & 1 & & \\
\hline (8) Total Assets & 0.106 & -0.015 & -0.008 & -0.018 & -0.008 & 0.008 & 0.062 & 1 & \\
\hline (9) Number of Workers & 0.078 & 0.006 & 0.005 & -0.001 & 0.004 & -0.006 & 0.079 & 0.286 & 1 \\
\hline
\end{tabular}


Table A1.aa: Spain: Descriptive Statistics

\begin{tabular}{lccccc}
\hline \hline & Obs. Number & Mean & Std. Dev. & Min & Max \\
\hline Listing (dummy) & 390890 & 0.001 & 0.034 & 0 & 1 \\
ROA (EBIT/TA, \%) & 390887 & 2.685 & 18.279 & -6808.85 & 639.13 \\
ROK(EBIT/FIXED ASSET, \%) & 382739 & 0.437 & 7.023 & -1923.5 & 669 \\
ROA (P/L before tax, \%) & 390289 & 1.686 & 11.417 & -99.95 & 99.97 \\
ROK & 382229 & 37.309 & 570.76 & -65573.758 & 63810.758 \\
D/A (ratio) & 390890 & 0.569 & 0.265 & -2.554 & 1 \\
Age & 390799 & 17.595 & 11.113 & 1 & 136 \\
Total Assets & 390890 & 4180.871 & 79059.894 & 1 & 13096668 \\
Number of Workers & 390890 & 15.979 & 148.927 & 1 & 26083 \\
\hline
\end{tabular}

Spain: Correlation Table

\begin{tabular}{|c|c|c|c|c|c|c|c|c|c|}
\hline & $\overline{(1)}$ & (2) & 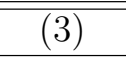 & $(4)$ & 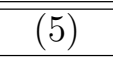 & 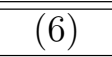 & 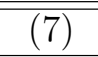 & $\overline{(8)}$ & 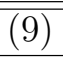 \\
\hline (1) Listing (dummy) & 1 & & & & & & & & \\
\hline (2) ROA (EBIT/TA, \%) & 0.002 & 1 & & & & & & & \\
\hline (3) ROK(EBIT/FIXED ASSET, \%) & -0.002 & 0.238 & 1 & & & & & & \\
\hline (4) ROA (P/L befor ) & 0.002 & 0.972 & 0.235 & 1 & & & & & \\
\hline (5) ROK & -0.002 & 0.234 & 0.939 & 0.243 & 1 & & & & \\
\hline (6) $\mathrm{D} / \mathrm{A}$ (ratio) & -0.007 & -0.051 & -0.022 & -0.117 & -0.035 & 1 & & & \\
\hline (7) Age & 0.102 & -0.089 & -0.04 & -0.07 & -0.036 & -0.283 & 1 & & \\
\hline (8) Total Assets & 0.477 & 0.008 & -0.002 & 0.009 & -0.001 & -0.005 & 0.08 & 1 & \\
\hline (9) Number of Workers & 0.484 & 0.015 & -0.002 & 0.015 & -0.002 & -0.007 & 0.096 & 0.843 & 1 \\
\hline
\end{tabular}

Table A1.ab: Sweden: Descriptive Statistics

\begin{tabular}{lccccc}
\hline \hline & Obs. Number & Mean & Std. Dev. & Min & Max \\
\hline Listing (dummy) & 40814 & 0.04 & 0.197 & 0 & 1 \\
ROA (EBIT/TA, \%) & 40799 & 8.304 & 18.532 & -471.311 & 852.632 \\
ROK(EBIT/FIXED ASSET, \%) & 39875 & 1.451 & 17.37 & -2360 & 595 \\
ROA (P/L before tax, \%) & 40684 & 7.827 & 16.059 & -99.82 & 99.32 \\
ROK & 39765 & 149.184 & 1287.455 & -94502.656 & 60239.188 \\
D/A (ratio) & 40814 & 0.541 & 0.236 & -0.538 & 0.999 \\
Age & 40798 & 21.569 & 17.851 & 1 & 327 \\
Total Assets & 40814 & 56157.783 & 1105597.744 & 9 & 53679911 \\
Number of Workers & 40814 & 169.829 & 2830.419 & 1 & 118055 \\
\hline
\end{tabular}


Sweden: Correlation Table

\begin{tabular}{|c|c|c|c|c|c|c|c|c|c|}
\hline & $(1)$ & 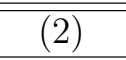 & $(3)$ & $(4)$ & $(5)$ & 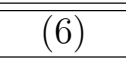 & $\overline{(7)}$ & $\overline{(8)}$ & 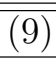 \\
\hline (1) Listing (dummy) & 1 & & & & & & & & \\
\hline (2) ROA (EBIT/TA, \%) & -0.236 & 1 & & & & & & & \\
\hline (3) ROK(EBIT/FIXED ASSET, \%) & -0.069 & 0.241 & 1 & & & & & & \\
\hline (4) $\mathrm{ROA}(\mathrm{P} / \mathrm{L}$ befor $)$ & -0.228 & 0.969 & 0.238 & 1 & & & & & \\
\hline (5) $\mathrm{ROK}$ & -0.069 & 0.236 & 0.984 & 0.245 & 1 & & & & \\
\hline (6) $\mathrm{D} / \mathrm{A}$ (ratio) & -0.073 & -0.193 & -0.054 & -0.266 & -0.064 & 1 & & & \\
\hline (7) Age & 0.148 & -0.054 & -0.025 & -0.037 & -0.021 & -0.164 & 1 & & \\
\hline (8) Total Assets & 0.242 & -0.007 & -0.006 & -0.007 & -0.005 & 0.016 & 0.214 & 1 & \\
\hline (9) Number of Workers & 0.276 & -0.005 & -0.006 & -0.005 & -0.006 & 0.018 & 0.247 & 0.938 & 1 \\
\hline
\end{tabular}

Table A1.ac: Taiwan: Descriptive Statistics

\begin{tabular}{lccccc}
\hline \hline & Obs. Number & Mean & Std. Dev. & Min & Max \\
\hline Listing (dummy) & 6998 & 0.945 & 0.227 & 0 & 1 \\
ROA (EBIT/TA, \%) & 6998 & 4.086 & 10.61 & -161.165 & 77.302 \\
ROK(EBIT/FIXED ASSET, \%) & 6997 & 0.172 & 1.255 & -69.615 & 24.315 \\
ROA (P/L before tax, \%) & 6991 & 3.135 & 11.402 & -94.52 & 96.61 \\
ROK & 6990 & 15.439 & 108.171 & -4307.175 & 2674.671 \\
D/A (ratio) & 6998 & 0.423 & 0.188 & 0.004 & 0.992 \\
Age & 6998 & 25.234 & 13.121 & 1 & 70 \\
Total Assets & 6998 & 549146.182 & 2373492.734 & 107 & 58437272 \\
Number of Workers & 6998 & 3573.246 & 24239.422 & 1 & 961000 \\
\hline
\end{tabular}

Taiwan: Correlation Table

\begin{tabular}{|c|c|c|c|c|c|c|c|c|c|}
\hline & $(1)$ & $(2)$ & $(3)$ & $(4)$ & $(5)$ & $(6)$ & $(7)$ & (8) & $(9)$ \\
\hline (1) Listing (dummy) & 1 & & & & & & & & \\
\hline (2) ROA (EBIT/TA, \%) & 0.024 & 1 & & & & & & & \\
\hline (3) ROK(EBIT/FIXED ASSET, \%) & 0.023 & 0.383 & 1 & & & & & & \\
\hline (4) ROA (P/L before tax, \%) & 0.003 & 0.914 & 0.337 & 1 & & & & & \\
\hline (5) $\mathrm{ROK}$ & 0.021 & 0.437 & 0.916 & 0.46 & 1 & & & & \\
\hline (6) $\mathrm{D} / \mathrm{A}$ (ratio) & -0.023 & -0.144 & -0.04 & -0.193 & -0.082 & 1 & & & \\
\hline (7) Age & -0.047 & 0.038 & -0.032 & 0.063 & -0.028 & 0.127 & 1 & & \\
\hline (8) Total Assets & 0.018 & 0.039 & -0.002 & 0.041 & 0 & 0.138 & 0.115 & 1 & \\
\hline (9) Number of Workers & 0.025 & 0.029 & 0.002 & 0.03 & 0.003 & 0.106 & 0.062 & 0.57 & 1 \\
\hline
\end{tabular}


Table A1.ad: US: Descriptive Statistics

\begin{tabular}{lccccc}
\hline \hline & Obs. Number & Mean & Std. Dev. & Min & Max \\
\hline Listing (dummy) & 8944 & 0.996 & 0.062 & 0 & 1 \\
ROA (EBIT/TA, \%) & 8944 & -138.258 & 11031.192 & -1031163.625 & 164.67 \\
ROK(EBIT/FIXED ASSET, \%) & 8924 & -9.292 & 191.582 & -12153.333 & 455.886 \\
ROA (P/L before tax, \%) & 8694 & -0.901 & 22.751 & -99.77 & 94.94 \\
ROK & 8688 & -695.377 & 18386.518 & -1267476.75 & 45592.902 \\
D/A (ratio) & 8944 & 0.451 & 0.232 & -0.464 & 1 \\
Age & 8944 & 29.529 & 27.526 & 1 & 141 \\
Total Assets & 8944 & 6550319.367 & 30993592.27 & 2 & 797769000 \\
Number of Workers & 8944 & 9826.254 & 26987.762 & 1 & 349600 \\
\hline
\end{tabular}

US: Correlation Table

\begin{tabular}{|c|c|c|c|c|c|c|c|c|c|}
\hline & $(1)$ & $(2)$ & $(3)$ & $(4)$ & $(5)$ & $(6)$ & $(7)$ & $(8)$ & $(9)$ \\
\hline (1) Listing (dummy) & 1 & & & & & & & & \\
\hline (2) ROA (EBIT/TA, \%) & -0.012 & 1 & & & & & & & \\
\hline (3) ROK(EBIT/FIXED ASSET, \%) & -0.002 & 0.117 & 1 & & & & & & \\
\hline (4) ROA (P/L befor ) & -0.01 & 0.911 & 0.123 & 1 & & & & & \\
\hline (5) $\mathrm{ROK}$ & -0.002 & 0.114 & 0.999 & 0.122 & 1 & & & & \\
\hline (6) $\mathrm{D} / \mathrm{A}$ (ratio) & -0.02 & 0.099 & 0.032 & 0.057 & 0.031 & 1 & & & \\
\hline (7) Age & 0.045 & 0.21 & 0.025 & 0.221 & 0.024 & 0.154 & 1 & & \\
\hline (8) Total Assets & 0.013 & 0.075 & 0.008 & 0.086 & 0.008 & 0.156 & 0.24 & 1 & \\
\hline (9) Number of Workers & 0.021 & 0.137 & 0.014 & 0.143 & 0.014 & 0.287 & 0.279 & 0.689 & 1 \\
\hline
\end{tabular}


Table A1.ae: Ukraine: Descriptive Statistics

\begin{tabular}{lccccc}
\hline \hline & Obs. Number & Mean & Std. Dev. & Min & Max \\
\hline Listing (dummy) & 147324 & 0.007 & 0.085 & 0 & 1 \\
ROA (EBIT/TA, \%) & 147324 & 7.93 & 269.697 & -39675 & 90700 \\
ROK(EBIT/FIXED ASSET, \%) & 125665 & 1.34 & 16.297 & -1705 & 2721 \\
ROA (P/L before tax, \%) & 144205 & 6.949 & 22.478 & -100 & 100 \\
ROK & 123897 & 121.769 & 1069.173 & -27575.592 & 148172.422 \\
D/A (ratio) & 147324 & 0.401 & 0.313 & -2.833 & 1 \\
Age & 137708 & 11.96 & 10.588 & 1 & 466 \\
Total Assets & 147324 & 1393.083 & 17869.065 & 1 & 1802272 \\
Number of Workers & 147324 & 43.902 & 280.702 & 1 & 27688 \\
\hline
\end{tabular}

Ukraine: Correlation Table

\begin{tabular}{|c|c|c|c|c|c|c|c|c|c|}
\hline & $\overline{(1)}$ & $\overline{(2)}$ & 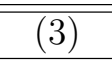 & $(4)$ & $(5)$ & $\overline{(6)}$ & $\overline{(7)}$ & $(8)$ & $\overline{(9)}$ \\
\hline (1) Listing (dummy) & 1 & & & & & & & & \\
\hline (2) ROA (EBIT/TA, \%) & -0.016 & 1 & & & & & & & \\
\hline (3) ROK(EBIT/FIXED ASSET, \%) & -0.009 & 0.209 & 1 & & & & & & \\
\hline (4) ROA (P/L befor ) & -0.021 & 0.988 & 0.21 & 1 & & & & & \\
\hline (5) $\mathrm{ROK}$ & -0.01 & 0.213 & 0.982 & 0.216 & 1 & & & & \\
\hline (6) $\mathrm{D} / \mathrm{A}$ (ratio) & 0.003 & -0.063 & 0.017 & -0.074 & 0.013 & 1 & & & \\
\hline (7) Age & 0.283 & -0.022 & -0.027 & -0.029 & -0.028 & -0.06 & 1 & & \\
\hline (8) Total Assets & 0.154 & -0.001 & 0.003 & -0.007 & -0.002 & 0.045 & 0.118 & 1 & \\
\hline (9) Number of Workers & 0.197 & 0.012 & -0.006 & 0.003 & -0.007 & 0.047 & 0.197 & 0.809 & 1 \\
\hline
\end{tabular}


Table A1.af: UK: Descriptive Statistics

\begin{tabular}{lccccc}
\hline \hline & Obs. Number & Mean & Std. Dev. & Min & Max \\
\hline Listing (dummy) & 23707 & 0.12 & 0.324 & 0 & 1 \\
ROA (EBIT/TA, \%) & 23698 & 7.273 & 32.981 & -806.305 & 2937.302 \\
ROK(EBIT/FIXED ASSET, \%) & 23363 & 0.718 & 38.466 & -1119.333 & 3826.667 \\
ROA (P/L before tax, \%) & 23439 & 6.969 & 15.843 & -98.78 & 100 \\
ROK & 23166 & 120.723 & 3860.978 & -102737.117 & 383348.531 \\
D/A (ratio) & 23707 & 0.498 & 0.238 & 0 & 1 \\
Age & 23703 & 29.144 & 27.057 & 1 & 218 \\
Total Assets & 23707 & 369223.241 & 6172565.771 & 1 & 305690000 \\
Number of Workers & 23707 & 706.743 & 5740.418 & 1 & 174381 \\
\hline
\end{tabular}

UK: Correlation Table

\begin{tabular}{|c|c|c|c|c|c|c|c|c|c|}
\hline & (1) & 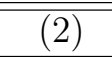 & (3) & (4) & $\overline{(5)}$ & 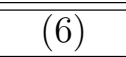 & 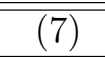 & $\overline{(8)}$ & 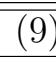 \\
\hline (1) Listing (dummy) & 1 & & & & & & & & \\
\hline (2) ROA (EBIT/TA, \%) & -0.22 & 1 & & & & & & & \\
\hline (3) ROK(EBIT/FIXED ASSET, \%) & -0.039 & 0.112 & 1 & & & & & & \\
\hline (4) $\mathrm{ROA}(\mathrm{P} / \mathrm{L}$ before tax $)$ & -0.248 & 0.898 & 0.117 & 1 & & & & & \\
\hline (5) $\mathrm{ROK}$ & -0.036 & 0.1 & 0.977 & 0.111 & 1 & & & & \\
\hline (6) $\mathrm{D} / \mathrm{A}$ (ratio) & -0.103 & 0.047 & 0.005 & -0.006 & 0.002 & 1 & & & \\
\hline (7) Age & 0.124 & -0.009 & -0.012 & 0 & -0.011 & -0.207 & 1 & & \\
\hline (8) Total Assets & 0.155 & 0.003 & -0.002 & 0.003 & -0.002 & 0.038 & 0.082 & 1 & \\
\hline (9) Number of Workers & 0.266 & 0.016 & -0.003 & 0.013 & -0.003 & 0.068 & 0.116 & 0.623 & 1 \\
\hline
\end{tabular}


Table A1.ag: Vietnam: Descriptive Statistics

\begin{tabular}{lccccc}
\hline \hline & Obs. Number & Mean & Std. Dev. & Min & Max \\
\hline Listing (dummy) & 70833 & 0.015 & 0.122 & 0 & 1 \\
ROA (EBIT/TA, \%) & 70833 & -0.915 & 58.961 & -11200 & 736.752 \\
ROK(EBIT/FIXED ASSET, \%) & 51389 & 0.152 & 6.347 & -109 & 743 \\
ROA (P/L before tax, \%) & 70576 & -0.833 & 9.75 & -99.92 & 99.38 \\
ROK & 51295 & 4.229 & 595.303 & -6997.44 & 70946.414 \\
D/A (ratio) & 70833 & 0.398 & 0.363 & -24.5 & 1 \\
Age & 70624 & 5.914 & 7.228 & 1 & 1002 \\
Total Assets & 70833 & 3639.419 & 39854.336 & -25 & 4260011 \\
Number of Workers & 70833 & 71.595 & 481.021 & 1 & 36904 \\
\hline
\end{tabular}

Vietnam: Correlation Table

\begin{tabular}{lccccccccc}
\hline \hline & $(1)$ & $(2)$ & $(3)$ & $(4)$ & $(5)$ & $(6)$ & $(7)$ & $(8)$ & $(9)$ \\
\hline (1) Listing (dummy) & 1 & & & & & & & & \\
(2) ROA (EBIT/TA, \%) & 0.111 & 1 & & & & & & \\
(3) ROK(EBIT/FIXED ASSET, \%) & 0.003 & 0.205 & 1 & & & & & \\
(4) ROA (P/L befor) & 0.115 & 0.876 & 0.182 & 1 & & & & \\
(5) ROK & 0.005 & 0.18 & 0.954 & 0.199 & 1 & & & \\
(6) D/A (ratio) & 0.017 & 0.086 & 0.026 & 0.028 & 0.013 & 1 & & \\
(7) Age & 0.355 & 0.179 & 0.024 & 0.17 & 0.024 & 0.128 & 1 & & \\
(8) Total Assets & 0.177 & 0.058 & 0.004 & 0.056 & 0.004 & 0.054 & 0.115 & 1 & \\
(9) Number of Workers & 0.24 & 0.098 & 0.003 & 0.09 & 0.004 & 0.062 & 0.197 & 0.37 & 1 \\
\hline
\end{tabular}




\section{Appendix A2. Propensity Score Match}

Table A2.1: Propensity Score Matching Estimates

Propensity scores for the listing probability are used to match the treated (i.e., the listed) to the controlled (i.e., the unlisted), based onone-to-one nearest neighbour matching restricting to the common support. Size (logarithm of total assets), Age(the years since incorporation), and Industry(2-digit level) areused to compute propensity scores. Differences (ATT) of MPK (ROA) between the treated and controlled are reported here. Upper Half refers to $50 \%<=$ Propensity Score $<=75 \%$ and the Lower Half refers to $50 \%<=$ Propensity Score

\begin{tabular}{|c|c|c|c|c|c|c|c|c|}
\hline & Parts & ATT & SE & t-stat & $\begin{array}{c}\text { Obs. } \\
\text { Untreated (on } \\
\text { support) } \\
\end{array}$ & $\begin{array}{l}\text { Obs. Treated } \\
\text { (on support) }\end{array}$ & $\begin{array}{c}\text { Obs. } \\
\text { Untreated } \\
\text { (off support) }\end{array}$ & $\begin{array}{l}\text { Obs. Treated } \\
\text { (off support) }\end{array}$ \\
\hline \multirow{3}{*}{ Austria } & Overall & -1.419 & 1.689 & $\begin{array}{l}-0.840 \\
\end{array}$ & 954 & 50 & 0 & 39 \\
\hline & Upper Half & 1.940 & 1.958 & 0.990 & 20 & 26 & 0 & 37 \\
\hline & Lower Half & -4.275 & 1.863 & -2.290 & 934 & 19 & 0 & 7 \\
\hline \multirow{3}{*}{ Australia } & Overall & -27.031 & 3.505 & -7.710 & 978 & 527 & 0 & 16 \\
\hline & Upper Half & -31.373 & 4.699 & -6.680 & 67 & 350 & 0 & 12 \\
\hline & Lower Half & -18.428 & 3.648 & -5.050 & 911 & 176 & 0 & 5 \\
\hline \multirow{3}{*}{ Belgium } & Overall & -4.082 & 1.943 & -2.100 & 44,999 & 225 & 0 & 22 \\
\hline & Upper Half & 0.866 & 1.247 & 0.690 & 38 & 111 & 0 & 27 \\
\hline & Lower Half & -9.560 & 2.965 & -3.220 & 44,961 & 108 & 0 & 1 \\
\hline \multirow{3}{*}{ Bulgaria } & Overall & -4.966 & 1.390 & -3.570 & 61,008 & 210 & 0 & 13 \\
\hline & Upper Half & -2.313 & 2.135 & -1.080 & 35 & 48 & 0 & 13 \\
\hline & Lower Half & -5.752 & 1.566 & -3.670 & 60,973 & 162 & 0 & 0 \\
\hline \multirow{3}{*}{ Brazil } & Overall & 3.250 & 3.978 & 0.820 & 414 & 13 & 0 & 17 \\
\hline & Upper Half & & & & & & & \\
\hline & Lower Half & 3.250 & 3.978 & 0.820 & 412 & 13 & 0 & 1 \\
\hline \multirow{3}{*}{ China } & Overall & -2.536 & 0.527 & -4.810 & 445,882 & 20,229 & 0 & 0 \\
\hline & Upper Half & -2.037 & 1.000 & -2.040 & 4,175 & 11,032 & 0 & 0 \\
\hline & Lower Half & -3.142 & 0.511 & -6.150 & 441,707 & 9,196 & 0 & 1 \\
\hline \multirow{3}{*}{ Croatia } & Overall & -7.213 & 0.905 & -7.970 & 33,934 & 282 & 0 & 71 \\
\hline & Upper Half & -6.201 & 1.894 & -3.270 & 77 & 72 & 0 & 71 \\
\hline & Lower Half & -7.547 & 0.953 & -7.920 & 33,857 & 210 & 0 & 0 \\
\hline & Overall & -2.606 & 1.682 & -1.550 & 36,671 & 51 & 0 & 15 \\
\hline \multirow[t]{2}{*}{ Czech Republi } & c Upper Half & 2.382 & 0.976 & 2.440 & 12 & 3 & 0 & 15 \\
\hline & Lower Half & -2.917 & 1.783 & -1.640 & 36,659 & 48 & 0 & 0 \\
\hline \multirow{3}{*}{ Denmark } & Overall & -4.475 & 2.476 & -1.810 & 2,381 & 133 & 0 & 76 \\
\hline & Upper Half & -2.141 & 2.278 & -0.940 & 46 & 92 & 0 & 72 \\
\hline & Lower Half & -10.088 & 5.676 & -1.780 & 2,335 & 37 & 0 & 8 \\
\hline \multirow{3}{*}{ Finland } & Overall & -0.728 & 1.182 & $\begin{array}{c}-0.620 \\
\end{array}$ & 30,390 & 438 & 0 & 46 \\
\hline & Upper Half & 0.217 & 1.283 & 0.170 & 54 & 330 & 0 & 67 \\
\hline & Lower Half & -6.162 & 2.260 & -2.730 & 30,336 & 83 & 0 & 4 \\
\hline
\end{tabular}


Table A2.2: Propensity Score Matching Estimates

Propensity scores for the listing probability are used to match the treated (i.e., the listed) to the controlled (i.e., the unlisted), based onone-to-one nearest neighbour matching restricting to the common support. Size (logarithm of total assets), Age(the years since incorporation), and Industry(2-digit level) areused to compute propensity scores. Differences (ATT) of MPK (ROA) between the treated and controlled are reported here. Upper Half refers to $50 \%<=$ Propensity Score $<=75 \%$ and the Lower Half refers to $50 \%<=$ Propensity Score

\begin{tabular}{|c|c|c|c|c|c|c|c|c|}
\hline & Parts & ATT & SE & t-stat & $\begin{array}{c}\text { Obs. } \\
\text { Untreated (on } \\
\text { support) } \\
\end{array}$ & $\begin{array}{l}\text { Obs. Treated } \\
\text { (on support) }\end{array}$ & $\begin{array}{c}\text { Obs. } \\
\text { Untreated } \\
\text { (off support) }\end{array}$ & $\begin{array}{l}\text { Obs. Treated } \\
\text { (off support) }\end{array}$ \\
\hline & Overall & -9.381 & 1.533 & -6.120 & 96,700 & 1,113 & 0 & 22 \\
\hline \multirow[t]{2}{*}{ France } & Upper Half & -1.485 & 1.904 & -0.780 & 164 & 649 & 0 & 57 \\
\hline & Lower Half & -22.397 & 2.169 & -10.330 & 96,536 & 428 & 0 & 1 \\
\hline & Overall & 0.351 & 0.617 & 0.570 & 25,914 & 597 & 0 & 2 \\
\hline \multirow[t]{2}{*}{ Greece } & Upper Half & 4.439 & 0.919 & 4.830 & 92 & 254 & 0 & 10 \\
\hline & Lower Half & 2.886 & 0.666 & -4.330 & 25,822 & 335 & 0 & 0 \\
\hline \multirow{3}{*}{ Germany } & Overall & -6.461 & 0.645 & -10.020 & 43,180 & 1,343 & 0 & 22 \\
\hline & Upper Half & -1.456 & 0.882 & -1.650 & 180 & 453 & 0 & 24 \\
\hline & Lower Half & -9.004 & 0.773 & -11.650 & 43,000 & 888 & 0 & 0 \\
\hline \multirow{3}{*}{ Hungary } & Overall & 4.673 & 1.503 & 3.110 & 40,598 & 55 & 0 & 14 \\
\hline & Upper Half & -0.016 & 4.429 & -0.000 & 9 & 3 & 0 & 14 \\
\hline & Lower Half & 4.967 & 1.593 & 3.120 & 40,589 & 51 & 0 & 1 \\
\hline \multirow{3}{*}{ Iceland } & Overall & 0.043 & 0.016 & 2.680 & 871 & 28 & 0 & 33 \\
\hline & Upper Half & 0.040 & 0.020 & 1.970 & 15 & 22 & 0 & 29 \\
\hline & Lower Half & 0.069 & 0.011 & 6.170 & 856 & 5 & 0 & 5 \\
\hline \multirow{3}{*}{ India } & Overall & -1.153 & 7.007 & -0.160 & 76 & 1,413 & 0 & 229 \\
\hline & Upper Half & -1.246 & 7.207 & -0.170 & 63 & 1,406 & 0 & 229 \\
\hline & Lower Half & 10.484 & 8.001 & 1.310 & 13 & 7 & 0 & 0 \\
\hline \multirow{3}{*}{ Ireland } & Overall & -22.559 & 4.706 & -4.790 & 1,629 & 115 & 0 & 111 \\
\hline & Upper Half & -21.233 & 4.820 & -4.410 & 12 & 87 & 0 & 113 \\
\hline & Lower Half & -28.519 & 8.891 & -3.210 & 1,617 & 24 & 0 & 2 \\
\hline \multirow{3}{*}{ Italy } & Overall & -2.807 & 0.790 & -3.550 & 439,632 & 378 & 0 & 10 \\
\hline & Upper Half & 0.093 & 0.886 & 0.100 & 37 & 115 & 0 & 12 \\
\hline & Lower Half & -4.119 & 0.970 & -4.250 & 439,595 & 261 & 0 & 0 \\
\hline \multirow{3}{*}{ Japan } & Overall & -0.330 & 0.254 & -1.300 & 169,676 & 12,579 & 0 & 9 \\
\hline & Upper Half & 0.262 & 0.314 & 0.830 & 1,539 & 9,267 & 0 & 11 \\
\hline & Lower Half & -1.975 & 0.260 & -7.590 & 168,137 & 3310 & 0 & 0 \\
\hline \multirow{3}{*}{ Korea } & Overall & -4.481 & 0.839 & -5.340 & 162,122 & 2,980 & 0 & 1 \\
\hline & Upper Half & -4.498 & 5.017 & -0.900 & 315 & 532 & 0 & 1 \\
\hline & Lower Half & -4.438 & 0.412 & -10.770 & 161,807 & 2,448 & 0 & 0 \\
\hline
\end{tabular}


Table A2.3: Propensity Score Matching Estimates

Propensity scores for the listing probability are used to match the treated (i.e., the listed) to the controlled (i.e., the unlisted), based onone-to-one nearest neighbour matching restricting to the common support. Size (logarithm of total assets), Age(the years since incorporation), and Industry(2-digit level) areused to compute propensity scores. Differences (ATT) of MPK (ROA) between the treated and controlled are reported here. Upper Half refers to $50 \%<=$ Propensity Score $<=75 \%$ and the Lower Half refers to

Obs.

Untreated (on Obs. Treated Untreated Obs. Treated

\begin{tabular}{|c|c|c|c|c|c|c|c|c|}
\hline & Parts & ATT & SE & t-stat & $\begin{array}{l}\text { Untreated (or } \\
\text { support) }\end{array}$ & $\begin{array}{l}\text { Obs. Treated } \\
\text { (on support) }\end{array}$ & $\begin{array}{c}\text { Untreated } \\
\text { (off support) }\end{array}$ & $\begin{array}{l}\text { Obs. Treated } \\
\text { (off support) }\end{array}$ \\
\hline \multirow{3}{*}{ Latvia } & Overall & -2.252 & 2.923 & -0.770 & 14,223 & 40 & 0 & 93 \\
\hline & Upper Half & 5.769 & 2.597 & 2.220 & 3 & 2 & 0 & 93 \\
\hline & Lower Half & -2.963 & 3.007 & -0.990 & 14,220 & 36 & 0 & 2 \\
\hline \multirow{3}{*}{ Malaysia } & Overall & 2.968 & 3.717 & 0.800 & 243 & 31 & 0 & 27 \\
\hline & Upper Half & 10.172 & 5.662 & 1.800 & 13 & 4 & 0 & 32 \\
\hline & Lower Half & 2.663 & 4.863 & 0.550 & 230 & 20 & 0 & 2 \\
\hline \multirow{3}{*}{ Netherlands } & Overall & -2.560 & 3.335 & -0.770 & 1,182 & 288 & 0 & 37 \\
\hline & Upper Half & -1.864 & 2.042 & -0.910 & 36 & 207 & 0 & 60 \\
\hline & Lower Half & -5.535 & 4.917 & -1.130 & 1,146 & 58 & 0 & 0 \\
\hline \multirow{3}{*}{ Norway } & Overall & -12.873 & 7.608 & -1.690 & 5,610 & 62 & 0 & 16 \\
\hline & Upper Half & -2.748 & 9.198 & -0.300 & 10 & 46 & 0 & 15 \\
\hline & Lower Half & -35.871 & 15.465 & -2.320 & 5,600 & 14 & 0 & 3 \\
\hline \multirow{3}{*}{ Poland } & Overall & -4.524 & 0.957 & -4.730 & 22,178 & 529 & 0 & 51 \\
\hline & Upper Half & -1.553 & 1.513 & -1.030 & 82 & 177 & 0 & 50 \\
\hline & Lower Half & -5.984 & 1.019 & -5.870 & 22,096 & 350 & 0 & 3 \\
\hline \multirow{3}{*}{ Romania } & Overall & $\begin{array}{l}-8.444 \\
\end{array}$ & 4.743 & -1.780 & 149,998 & 935 & 0 & 82 \\
\hline & Upper Half & 24.611 & 44.403 & 0.550 & 8 & 29 & 0 & 104 \\
\hline & Lower Half & 12.264 & 4.981 & -2.460 & 149,990 & 884 & 0 & 0 \\
\hline \multirow{3}{*}{ Spain } & Overall & $\begin{array}{l}-2.838 \\
\end{array}$ & 0.803 & -3.540 & 323,691 & 443 & 0 & 7 \\
\hline & Upper Half & -3.163 & 1.543 & -2.050 & 66 & 207 & 0 & 7 \\
\hline & Lower Half & -2.510 & 0.705 & -3.560 & 323,625 & 235 & 0 & 1 \\
\hline \multirow{3}{*}{ Sweden } & Overall & -26.453 & 1.462 & -18.09 & 37,604 & 1,570 & 0 & 31 \\
\hline & Upper Half & -14.255 & 1.8889291 & -7.55 & 206 & 803 & 0 & 63 \\
\hline & Lower Half & -40.954 & 2.009 & -20.39 & 37,398 & 735 & 0 & 0 \\
\hline \multirow{3}{*}{ Taiwan } & Overall & -1.173 & 1.027 & -1.14 & 383 & 6,130 & 0 & 18 \\
\hline & Upper Half & -1.177 & 1.038 & -1.130 & 359 & 6120 & 0 & 18 \\
\hline & Lower Half & 1.226 & 4.695 & 0.260 & 24 & 10 & 0 & 0 \\
\hline \multirow[b]{2}{*}{ US } & Overall & -21.075 & 4.530 & -4.650 & 34 & 1,065 & 0 & 193 \\
\hline & $\begin{array}{l}\text { Upper Half } \\
\text { Lower Half }\end{array}$ & -21.075 & 4.530 & -4.650 & 34 & 1,065 & 0 & 193 \\
\hline \multirow{3}{*}{ Ukaraine } & Overall & -5.948 & 0.767 & -7.760 & 125,547 & 1,047 & 0 & 10 \\
\hline & Upper Half & -0.066 & 1.813 & -0.040 & 125 & 76 & 0 & 10 \\
\hline & Lower Half & -6.408 & 0.817 & -7.840 & 125,422 & 971 & 0 & 0 \\
\hline \multirow{3}{*}{ UK } & Overall & -16.808 & 1.205 & -13.940 & 20,797 & 2,793 & 0 & 34 \\
\hline & Upper Half & -5.614 & 2.523 & -2.220 & 337 & 1,216 & 0 & 34 \\
\hline & Lower Half & -25.443 & 1.475 & -17.250 & 20,460 & 1,569 & 0 & 8 \\
\hline \multirow{3}{*}{ Vietnam } & Overall & 1.530 & 0.480 & 3.190 & 69,446 & 1,006 & 0 & 1 \\
\hline & Upper Half & 2.483 & 0.996 & 2.490 & 225 & 344 & 0 & 2 \\
\hline & Lower Half & 1.071 & 0.531 & 2.020 & 69,221 & 661 & 0 & 0 \\
\hline
\end{tabular}


Table A2.4: Correlation between the ATT Estimates and the Fixed Effects Coefficients

\begin{tabular}{lccccc}
\hline & ATT ROA & ATT ROA Upper Half & ATT ROK & ATT ROK Upper Half & Fixed Effect Coeff \\
\hline \hline ATT ROA & 1 & & & & \\
ATT ROA Upper Half & 0.861 & 1 & & & \\
ATT ROK & 0.595 & 0.620 & 1 & & \\
ATT ROK Upper Half & 0.569 & 0.654 & 0.979 & 1 & \\
Fixed Effect Coeff & 0.614 & 0.318 & 0.106 & 0.046 & 1 \\
\hline
\end{tabular}


Appendix A3. Countrywise Benchmark Panel Regressions 
Table A3.1: Benchmark Panel Regressions for MPK

The dependent variable is MPK, proxied by ROA or ROK. Listing is the binary variable, taking the vlaue of one if a firm is listed and zero otherise. L.Size is the logarithm of the lagged total assets. Age is the years since incorporation. L.D/A is the lagged debt to asset ratio. 2-digit industry fixed effects are included but not reported in the results. The robust standard errors are reported in the

\begin{tabular}{|c|c|c|c|c|c|c|c|c|c|c|c|c|}
\hline & \multicolumn{4}{|c|}{ Australia } & \multicolumn{4}{|c|}{ Austria } & \multicolumn{4}{|c|}{ Belgium } \\
\hline & ROA(EBIT) & ROK(EBIT) & ROA(P/L) & ROK & ROA(EBIT) & ROK(EBIT) & ROA(P/L) & ROK & ROA(EBIT) & ROK(EBIT) & $\operatorname{ROA}(\mathbf{P} / \mathrm{L})$ & ROK \\
\hline Listing (dummy) & $\begin{array}{c}-11.600^{* * * *} \\
(-3.990)\end{array}$ & $\begin{array}{c}-2.593 \\
(-1.090)\end{array}$ & $\begin{array}{c}-9.095 * * * \\
(-5.130)\end{array}$ & $\begin{array}{l}36.150 \\
(0.220)\end{array}$ & $\begin{array}{c}-9.298 * * * \\
(-3.350)\end{array}$ & $\begin{array}{l}-0.965^{*} \\
(-2.390)\end{array}$ & $\begin{array}{c}-5.655^{* * * *} \\
(-3.480)\end{array}$ & $\begin{array}{c}-96.090^{*} \\
(-2.340)\end{array}$ & $\begin{array}{c}-2.833^{* *} \\
(-3.010)\end{array}$ & $\begin{array}{c}-0.570 \\
(-0.970)\end{array}$ & $\begin{array}{c}-4.939 * * * \\
(-5.800)\end{array}$ & $\begin{array}{l}-73.410 \\
(-1.150)\end{array}$ \\
\hline L.Size & $\begin{array}{c}5.137^{* * * *} \\
(7.910)\end{array}$ & $\begin{array}{c}2.432^{* * * *} \\
(4.580)\end{array}$ & $\begin{array}{c}2.259 * * * \\
(5.550)\end{array}$ & $\begin{array}{l}82.780^{*} \\
(2.210)\end{array}$ & $\begin{array}{c}1.992^{* * * *} \\
(4.080)\end{array}$ & $\begin{array}{c}0.125 \\
(1.760)\end{array}$ & $\begin{array}{l}0.809 * * \\
(2.820)\end{array}$ & $\begin{array}{l}11.690 \\
(1.610)\end{array}$ & $\begin{array}{c}0.195 * * * \\
(4.370)\end{array}$ & $\begin{array}{c}0.054 \\
(1.910)\end{array}$ & $\begin{array}{c}0.333^{* * *} \\
(8.250)\end{array}$ & $\begin{array}{l}7.123^{*} \\
(2.330)\end{array}$ \\
\hline Age & $\begin{array}{l}0.095^{*} \\
(2.330)\end{array}$ & $\begin{array}{c}0.004 \\
(0.101)\end{array}$ & $\begin{array}{c}0.101 * * * \\
(4.040)\end{array}$ & $\begin{array}{c}-0.464 \\
(-0.200)\end{array}$ & $\begin{array}{l}-0.012 \\
(-1.140)\end{array}$ & $\begin{array}{l}-0.001 \\
(-0.550)\end{array}$ & $\begin{array}{c}-0.020 * * * \\
(-3.310)\end{array}$ & $\begin{array}{c}-0.100 \\
(-0.650)\end{array}$ & $\begin{array}{c}-0.092 * * * \\
(-23.190)\end{array}$ & $\begin{array}{c}-0.017 * * * \\
(-6.770)\end{array}$ & $\begin{array}{c}-0.092 * * * \\
(-25.470)\end{array}$ & $\begin{array}{c}-1.655^{* * *} \\
(-6.110)\end{array}$ \\
\hline L.D/A (ratio) & $\begin{array}{c}1.757 \\
(0.370)\end{array}$ & $\begin{array}{l}-6.248 \\
(-1.600)\end{array}$ & $\begin{array}{c}0.496 \\
(0.170)\end{array}$ & $\begin{array}{l}-466.0 \\
(-1.720)\end{array}$ & $\begin{array}{l}-4.563^{*} \\
(-2.090)\end{array}$ & $\begin{array}{l}-0.751^{*} \\
(-2.360)\end{array}$ & $\begin{array}{c}-9.914 * * * \\
(-7.740)\end{array}$ & $\begin{array}{c}-88.660 * * \\
(-2.740)\end{array}$ & $\begin{array}{c}0.354 \\
(1.490)\end{array}$ & $\begin{array}{c}-0.738 * * * \\
(-4.930)\end{array}$ & $\begin{array}{l}-4.160 * * * \\
(-19.390)\end{array}$ & $\begin{array}{c}-135.300^{* * * *} \\
(-8.360)\end{array}$ \\
\hline Constant & $\begin{array}{c}-77.170^{* * *} \\
(-9.110) \\
\end{array}$ & $\begin{array}{c}-22.170 * * \\
(-3.210) \\
\end{array}$ & $\begin{array}{c}-44.130^{* * * *} \\
(-8.270)\end{array}$ & $\begin{array}{c}-849.0 \\
(-1.730) \\
\end{array}$ & $\begin{array}{c}-3.851 \\
(-0.220) \\
\end{array}$ & $\begin{array}{c}0.193 \\
(0.080) \\
\end{array}$ & $\begin{array}{l}12.130 \\
(1.190) \\
\end{array}$ & $\begin{array}{l}41.930 \\
(0.160) \\
\end{array}$ & $\begin{array}{c}7.315 \\
(0.580) \\
\end{array}$ & $\begin{array}{c}6.684 \\
(0.840) \\
\end{array}$ & $\begin{array}{c}8.898 \\
(0.780) \\
\end{array}$ & $\begin{array}{r}686.100 \\
(0.800) \\
\end{array}$ \\
\hline Observations & 1192 & 1187 & 1175 & 1170 & 1337 & 1337 & 1335 & 1335 & 51852 & 51267 & 51811 & 51231 \\
\hline Adjusted R-squared & 0.190 & 0.011 & 0.270 & -0.043 & -0.037 & 0.012 & 0.062 & 0.013 & 0.024 & 0.001 & 0.032 & 0.003 \\
\hline
\end{tabular}


Table A3.2: Benchmark Panel Regressions for MPK

The dependent variable is MPK, proxied by ROA or ROK. Listing is the binary variable, taking the vlaue of one if a firm is listed and zero otherise. L.Size is the logarithm of the lagged total assets. Age is the years since incorporation. L.D/A is the lagged debt to asset ratio. 2-digit industry fixed effects are included but not reported in the results. The robust standard errors are reported in the

\begin{tabular}{lcccccccccccc}
\multicolumn{10}{c}{ parenthesis corrected for clustering at the industry level: *denotes significance at $10 \% ; * *$ at $5 \%$; and $* * *$ at $1 \%$} \\
\hline \hline
\end{tabular}


Table A3.3: Benchmark Panel Regressions for MPK

The dependent variable is MPK, proxied by ROA or ROK. Listing is the binary variable, taking the vlaue of one if a firm is listed and zero otherise. L.Size is the logarithm of the lagged total assets. Age is the years since incorporation. L.D/A is the lagged debt to asset ratio. 2-digit industry fixed effects are included but not reported in the results. The robust standard errors are reported in the

\begin{tabular}{lcccccccccccc}
\multicolumn{10}{c}{ parenthesis corrected for clustering at the industry level: *denotes significance at $10 \%$; ** at $5 \%$; and $* * *$ at $1 \%$} \\
\hline \hline
\end{tabular}


Table A3.4: Benchmark Panel Regressions for MPK

The dependent variable is MPK, proxied by ROA or ROK. Listing is the binary variable, taking the vlaue of one if a firm is listed and zero otherise. L.Size is the logarithm of the lagged total assets. Age is the years since incorporation. L.D/A is the lagged debt to asset ratio. 2-digit industry fixed effects are included but not reported in the results. The robust standard errors are reported in the

\begin{tabular}{|c|c|c|c|c|c|c|c|c|c|c|c|c|}
\hline & \multicolumn{4}{|c|}{ Finland } & \multicolumn{4}{|c|}{ France } & \multicolumn{4}{|c|}{$\begin{array}{l}\text { Greece } \\
\end{array}$} \\
\hline & ROA(EBIT) & ROK(EBIT) & $\operatorname{ROA}(\mathbf{P} / \mathbf{L})$ & ROK & ROA(EBIT) & ROK(EBIT) & ROA(P/L) & ROK & ROA(EBIT) & ROK(EBIT) & $\operatorname{ROA}(\mathbf{P} / \mathbf{L})$ & ROK \\
\hline Listing (dummy) & $\begin{array}{c}-3.583 * * \\
(-2.660)\end{array}$ & $\begin{array}{c}-0.187 \\
(-0.520)\end{array}$ & $\begin{array}{l}-2.106^{*} \\
(-2.270)\end{array}$ & $\begin{array}{l}-26.210 \\
(-0.720)\end{array}$ & $\begin{array}{c}-8.743 * * * \\
(-8.950)\end{array}$ & $\begin{array}{c}-6.951 * * * \\
(-4.410)\end{array}$ & $\begin{array}{c}-8.359 * * * \\
(-17.040)\end{array}$ & $\begin{array}{c}-189.3 * * * \\
(-10.32)\end{array}$ & $\begin{array}{c}-1.528 * * \\
(-2.89)\end{array}$ & $\begin{array}{l}0.477 * \\
(2.12)\end{array}$ & $\begin{array}{c}-2.089 * * * \\
(-4.80)\end{array}$ & $\begin{array}{l}49.90^{*} \\
(2.20)\end{array}$ \\
\hline L.Size & $\begin{array}{c}0.719^{* * * *} \\
(7.490)\end{array}$ & $\begin{array}{l}-0.004 \\
(-0.160)\end{array}$ & $\begin{array}{c}0.417^{* * * *} \\
(6.290)\end{array}$ & $\begin{array}{c}2.072 \\
(0.770)\end{array}$ & $\begin{array}{l}0.269 * * \\
(3.210)\end{array}$ & $\begin{array}{c}0.958^{* * *} \\
(7.030)\end{array}$ & $\begin{array}{c}0.155^{* * *} \\
(3.700)\end{array}$ & $\begin{array}{l}2.101 \\
(1.33)\end{array}$ & $\begin{array}{r}-0.0637 \\
(-1.01)\end{array}$ & $\begin{array}{c}-0.339 * * * \\
(-12.60)\end{array}$ & $\begin{array}{c}-0.268^{* * * *} \\
(-5.17)\end{array}$ & $\begin{array}{r}-30.49 * * * \\
(-11.23)\end{array}$ \\
\hline Age & $\begin{array}{c}-0.094 * * * \\
(-8.760)\end{array}$ & $\begin{array}{c}-0.018^{* * * *} \\
(-6.200)\end{array}$ & $\begin{array}{c}-0.087 * * * \\
(-11.830)\end{array}$ & $\begin{array}{c}-1.962 * * * \\
(-6.780)\end{array}$ & $\begin{array}{c}-0.077 * * * \\
(-10.520)\end{array}$ & $\begin{array}{c}-0.023 \\
(-1.930)\end{array}$ & $\begin{array}{c}-0.0710^{* * *} \\
(-19.44)\end{array}$ & $\begin{array}{c}-0.731 * * * \\
(-5.35)\end{array}$ & $\begin{array}{c}-0.0105^{*} \\
(-2.26)\end{array}$ & $\begin{array}{c}-0.00375 \\
(-1.90)\end{array}$ & $\begin{array}{c}-0.0139 * * * \\
(-3.63)\end{array}$ & $\begin{array}{l}-0.380 \\
(-1.90)\end{array}$ \\
\hline L.D/A (ratio) & $\begin{array}{l}-1.530^{* *} \\
(-2.600)\end{array}$ & $\begin{array}{c}-1.252^{* * *} \\
(-7.910)\end{array}$ & $\begin{array}{l}-5.704 * * * \\
(-14.060)\end{array}$ & $\begin{array}{c}158.900^{* *} \\
(-9.880)\end{array}$ & $\begin{array}{c}-1.425^{* *} \\
(-2.860)\end{array}$ & $\begin{array}{c}3.118^{* * * *} \\
(3.870)\end{array}$ & $\begin{array}{c}-5.071 * * * \\
(-20.44)\end{array}$ & $\begin{array}{c}-98.15 * * * \\
(-10.53)\end{array}$ & $\begin{array}{c}3.075^{* * * *} \\
(10.07)\end{array}$ & $\begin{array}{c}0.915 * * * \\
(7.05)\end{array}$ & $\begin{array}{c}-1.129 * * * \\
(-4.49)\end{array}$ & $\begin{array}{c}40.97^{* *} \\
(3.12)\end{array}$ \\
\hline Constant & $\begin{array}{c}74.690^{* *} \\
(3.150)\end{array}$ & $\begin{array}{l}12.720^{*} \\
(2.030)\end{array}$ & $\begin{array}{c}77.940^{* * *} \\
(4.780)\end{array}$ & $\begin{array}{c}1270.80^{*} \\
(2.000)\end{array}$ & $\begin{array}{c}6.477 \\
(0.430)\end{array}$ & $\begin{array}{l}-5.352 \\
(-0.220)\end{array}$ & $\begin{array}{l}9.971 \\
(1.34)\end{array}$ & $\begin{array}{l}162.3 \\
(0.59)\end{array}$ & $\begin{array}{c}3.508^{* * * *} \\
(5.15)\end{array}$ & $\begin{array}{c}2.800^{* * * *} \\
(9.64)\end{array}$ & $\begin{array}{c}6.091^{* * *} \\
(10.86)\end{array}$ & $\begin{array}{c}271.2^{* * * *} \\
(9.25)\end{array}$ \\
\hline Observations & 22748 & 22110 & 22691 & 22077 & 62809 & 62318 & 62759 & 62271 & 22722 & 22655 & 22713 & 22650 \\
\hline Adjusted R-squared & 0.010 & 0.013 & 0.025 & 0.014 & 0.009 & -0.001 & 0.035 & 0.013 & 0.038 & 0.021 & 0.051 & 0.015 \\
\hline
\end{tabular}


Table A3.5: Benchmark Panel Regressions for MPK

The dependent variable is MPK, proxied by ROA or ROK. Listing is the binary variable, taking the vlaue of one if a firm is listed and zero otherise. L.Size is the logarithm of the lagged total assets. Age is the years since incorporation. L.D/A is the lagged debt to asset ratio. 2-digit industry fixed effects are included but not reported in the results. The robust standard errors are reported in the

\begin{tabular}{|c|c|c|c|c|c|c|c|c|c|c|c|c|}
\hline & \multicolumn{4}{|c|}{ 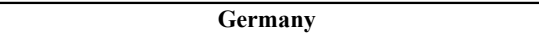 } & \multicolumn{4}{|c|}{ Hungary } & \multicolumn{4}{|c|}{ Iceland } \\
\hline & ROA(EBIT) & ROK(EBIT) & $\operatorname{ROA}(\mathbf{P} / \mathrm{L})$ & ROK & ROA(EBIT) & ROK(EBIT) & $\operatorname{ROA}(\mathbf{P} / \mathrm{L})$ & ROK & ROA(EBIT) & ROK(EBIT) & $\operatorname{ROA}(\mathbf{P} / \mathrm{L})$ & ROK \\
\hline Listing (dummy) & $\begin{array}{c}-7.182 * * * \\
(-5.50)\end{array}$ & $\begin{array}{l}-2.433 \\
(-1.63)\end{array}$ & $\begin{array}{c}-6.199 * * * \\
(-16.26)\end{array}$ & $\begin{array}{l}-233.1 \\
(-1.71)\end{array}$ & $\begin{array}{l}7.645^{*} \\
(2.07)\end{array}$ & $\begin{array}{l}1.262 \\
(1.44)\end{array}$ & $\begin{array}{c}8.539 * * * \\
(3.75)\end{array}$ & $\begin{array}{l}124.7 \\
(1.47)\end{array}$ & $\begin{array}{l}2.765 \\
(0.77)\end{array}$ & $\begin{array}{l}0.147 \\
(0.14)\end{array}$ & $\begin{array}{l}3.422 \\
(0.98)\end{array}$ & $\begin{array}{l}8.901 \\
(0.09)\end{array}$ \\
\hline L.Size & $\begin{array}{l}-0.0264 \\
(-0.15)\end{array}$ & $\begin{array}{l}0.133 \\
(0.65)\end{array}$ & $\begin{array}{c}-0.248 * * * \\
(-5.33)\end{array}$ & $\begin{array}{l}11.50 \\
(0.68)\end{array}$ & $\begin{array}{c}0.192 * * * \\
(3.53)\end{array}$ & $\begin{array}{c}-0.0929 * * * \\
(-6.73)\end{array}$ & $\begin{array}{c}-0.237 * * * \\
(-7.01)\end{array}$ & $\begin{array}{c}-9.449 * * * \\
(-7.08)\end{array}$ & $\begin{array}{c}-1.205^{* * * *} \\
(-3.46)\end{array}$ & $\begin{array}{c}-0.351^{* * * *} \\
(-3.43)\end{array}$ & $\begin{array}{c}-1.280 * * * \\
(-3.81)\end{array}$ & $\begin{array}{c}-31.32 * * \\
(-3.13)\end{array}$ \\
\hline Age & $\begin{array}{c}-0.00883 \\
(-1.76)\end{array}$ & $\begin{array}{c}-0.0124 * \\
(-2.17)\end{array}$ & $\begin{array}{c}-0.00597 * * * \\
\quad(-4.12)\end{array}$ & $\begin{array}{l}-1.111^{*} \\
(-2.14)\end{array}$ & $\begin{array}{c}-0.247^{* * * *} \\
(-19.63)\end{array}$ & $\begin{array}{c}-0.0358^{* * *} \\
(-11.66)\end{array}$ & $\begin{array}{c}-0.228 * * * \\
(-29.31)\end{array}$ & $\begin{array}{c}-3.533 * * * \\
(-11.90)\end{array}$ & $\begin{array}{c}-0.0120 \\
(-0.27)\end{array}$ & $\begin{array}{c}0.00270 \\
(0.21)\end{array}$ & $\begin{array}{c}0.0230 \\
(0.55)\end{array}$ & $\begin{array}{l}0.404 \\
(0.32)\end{array}$ \\
\hline L.D/A (ratio) & $\begin{array}{l}-1.023 \\
(-1.09)\end{array}$ & $\begin{array}{l}1.007 \\
(0.93)\end{array}$ & $\begin{array}{c}-5.669^{* * *} \\
(-21.62)\end{array}$ & $\begin{array}{l}0.523 \\
(0.01)\end{array}$ & $\begin{array}{c}2.629 * * * \\
(9.96)\end{array}$ & $\begin{array}{c}0.443 * * * \\
(6.91)\end{array}$ & $\begin{array}{c}0.637^{* * *} \\
(3.91)\end{array}$ & $\begin{array}{c}31.71 * * * \\
(5.12)\end{array}$ & $\begin{array}{l}1.041 \\
(0.50)\end{array}$ & $\begin{array}{l}-1.108 \\
(-1.79)\end{array}$ & $\begin{array}{c}-5.513^{* *} \\
(-2.74)\end{array}$ & $\begin{array}{l}-144.1 * \\
(-2.38)\end{array}$ \\
\hline Constant & $\begin{array}{l}9.504 \\
(0.34) \\
\end{array}$ & $\begin{array}{l}-1.676 \\
(-0.05) \\
\end{array}$ & $\begin{array}{l}12.53 \\
(1.51) \\
\end{array}$ & $\begin{array}{l}-84.32 \\
(-0.03) \\
\end{array}$ & $\begin{array}{r}1.446 \\
(0.05) \\
\end{array}$ & $\begin{array}{r}1.425 \\
(0.21) \\
\end{array}$ & $\begin{array}{l}5.817 \\
(0.34) \\
\end{array}$ & $\begin{array}{l}145.6 \\
(0.23) \\
\end{array}$ & $\begin{array}{c}26.40 * * * \\
(5.16) \\
\end{array}$ & $\begin{array}{c}7.111 \text { *** } \\
(4.42) \\
\end{array}$ & $\begin{array}{c}27.93 * * * \\
(5.66) \\
\end{array}$ & $\begin{array}{c}676.8^{* * *} \\
(4.32) \\
\end{array}$ \\
\hline Observations & 34162 & 33864 & 37646 & 37349 & 93164 & 87711 & 92730 & 87416 & 1672 & 1589 & 1668 & 1586 \\
\hline Adjusted R-squared & 0.001 & -0.001 & 0.037 & -0.001 & 0.011 & 0.005 & 0.028 & 0.006 & 0.045 & 0.122 & 0.049 & 0.119 \\
\hline
\end{tabular}


Table A3.6: Benchmark Panel Regressions for MPK

The dependent variable is MPK, proxied by ROA or ROK. Listing is the binary variable, taking the vlaue of one if a firm is listed and zero otherise. L.Size is the logarithm of the lagged total assets. Age is the years since incorporation. L.D/A is the lagged debt to asset ratio. 2-digit industry fixed effects are included but not reported in the results. The robust standard errors are reported in the

parenthesis corrected for clustering at the industry level: *denotes significance at $10 \% \cdot * *$ at $5 \% \cdot$ and $* * *$ at $1 \%$

\begin{tabular}{|c|c|c|c|c|c|c|c|c|c|c|c|c|}
\hline & \multicolumn{4}{|c|}{ India } & \multicolumn{4}{|c|}{ Ireland } & \multicolumn{4}{|c|}{ Italy } \\
\hline & ROA(EBIT) & ROK(EBIT) & $\operatorname{ROA}(\mathbf{P} / \mathrm{L})$ & ROK & ROA(EBIT) & ROK(EBIT) & $\operatorname{ROA}(\mathbf{P} / \mathrm{L})$ & ROK & ROA(EBIT) & ROK(EBIT) & $\operatorname{ROA}(\mathbf{P} / \mathbf{L})$ & ROK \\
\hline Listing (dummy) & $\begin{array}{l}0.396 \\
(0.25)\end{array}$ & $\begin{array}{l}-0.192 \\
(-0.44)\end{array}$ & $\begin{array}{l}-0.0995 \\
(-0.06)\end{array}$ & $\begin{array}{l}-60.00 \\
(-0.71)\end{array}$ & $\begin{array}{c}-5.930 * * \\
(-3.02)\end{array}$ & $\begin{array}{l}-3.999^{*} \\
(-1.99)\end{array}$ & $\begin{array}{c}-4.513^{* *} \\
(-2.95)\end{array}$ & $\begin{array}{l}-368.2 \\
(-1.54)\end{array}$ & $\begin{array}{l}0.0383 \\
(0.04)\end{array}$ & $\begin{array}{c}0.0848 \\
(0.22)\end{array}$ & $\begin{array}{l}-0.158 \\
(-0.32)\end{array}$ & $\begin{array}{l}2.157 \\
(0.06)\end{array}$ \\
\hline L.Size & $\begin{array}{c}0.828 * * * \\
(6.77)\end{array}$ & $\begin{array}{c}-0.0109 \\
(-0.33)\end{array}$ & $\begin{array}{c}0.686^{* * * *} \\
(5.60)\end{array}$ & $\begin{array}{l}4.950 \\
(0.76)\end{array}$ & $\begin{array}{c}0.984 * * * \\
(4.62)\end{array}$ & $\begin{array}{l}0.154 \\
(0.69)\end{array}$ & $\begin{array}{c}0.619^{* * *} \\
(3.35)\end{array}$ & $\begin{array}{l}6.198 \\
(0.21)\end{array}$ & $\begin{array}{c}-0.241^{* * *} \\
(-12.43)\end{array}$ & $\begin{array}{c}-0.169^{* * *} \\
(-19.65)\end{array}$ & $\begin{array}{c}-0.241^{* * *} \\
(-21.69)\end{array}$ & $\begin{array}{c}-14.17^{* * *} \\
(-16.72)\end{array}$ \\
\hline Age & $\begin{array}{l}-0.0207 \\
(-1.87)\end{array}$ & $\begin{array}{c}-0.000958 \\
(-0.32)\end{array}$ & $\begin{array}{c}-0.00336 \\
(-0.31)\end{array}$ & $\begin{array}{l}0.207 \\
(0.35)\end{array}$ & $\begin{array}{c}-0.00507 \\
(-0.23)\end{array}$ & $\begin{array}{r}-0.0220 \\
(-1.01)\end{array}$ & $\begin{array}{c}-0.0291 \\
(-1.76)\end{array}$ & $\begin{array}{l}-2.597 \\
(-1.02)\end{array}$ & $\begin{array}{c}-0.0725 * * * \\
(-37.52)\end{array}$ & $\begin{array}{c}-0.0159 * * * \\
(-18.69)\end{array}$ & $\begin{array}{c}-0.0762 * * * \\
(-68.97)\end{array}$ & $\begin{array}{c}-1.675^{* * *} \\
(-19.97)\end{array}$ \\
\hline L.D/A (ratio) & $\begin{array}{c}-3.324^{* *} \\
(-2.74)\end{array}$ & $\begin{array}{c}0.0818 \\
(0.25)\end{array}$ & $\begin{array}{c}-12.60^{* * *} \\
(-10.36)\end{array}$ & $\begin{array}{l}-22.37 \\
(-0.35)\end{array}$ & $\begin{array}{c}4.257 * * \\
(2.68)\end{array}$ & $\begin{array}{l}0.213 \\
(0.13)\end{array}$ & $\begin{array}{l}1.162 \\
(0.86)\end{array}$ & $\begin{array}{l}15.31 \\
(0.07)\end{array}$ & $\begin{array}{c}-6.125^{* * * *} \\
(-52.97)\end{array}$ & $\begin{array}{c}-1.406 * * * \\
(-27.56)\end{array}$ & $\begin{array}{c}-9.026 * * * \\
(-136.39)\end{array}$ & $\begin{array}{c}-180.6^{* * * *} \\
(-35.89)\end{array}$ \\
\hline Constant & $\begin{array}{l}-3.009 \\
(-0.33) \\
\end{array}$ & $\begin{array}{l}0.304 \\
(0.12) \\
\end{array}$ & $\begin{array}{c}-0.0588 \\
(-0.01) \\
\end{array}$ & $\begin{array}{l}26.78 \\
(0.06) \\
\end{array}$ & $\begin{array}{l}-13.75 \\
(-1.41) \\
\end{array}$ & $\begin{array}{l}2.765 \\
(0.28) \\
\end{array}$ & $\begin{array}{l}-5.226 \\
(-0.72) \\
\end{array}$ & $\begin{array}{l}356.7 \\
(0.32) \\
\end{array}$ & $\begin{array}{c}12.62 * * * \\
(3.69) \\
\end{array}$ & $\begin{array}{l}2.398 \\
(1.60) \\
\end{array}$ & $\begin{array}{c}12.61 * * * \\
(6.45)\end{array}$ & $\begin{array}{l}174.2 \\
(1.18) \\
\end{array}$ \\
\hline Observations & 1428 & 1426 & 1426 & 1424 & 1786 & 1744 & 1431 & 1401 & 425534 & 423162 & 425437 & 423071 \\
\hline Adjusted R-squared & 0.094 & -0.077 & 0.175 & -0.081 & 0.027 & -0.017 & 0.063 & -0.042 & 0.016 & 0.006 & 0.069 & 0.006 \\
\hline
\end{tabular}


Table A3.7: Benchmark Panel Regressions for MPK

The dependent variable is MPK, proxied by ROA or ROK. Listing is the binary variable, taking the vlaue of one if a firm is listed and zero otherise. L.Size is the logarithm of the lagged total assets. Age is the years since incorporation. L.D/A is the lagged debt to asset ratio. 2-digit industry fixed effects are included but not reported in the results. The robust standard errors are reported in the

\begin{tabular}{|c|c|c|c|c|c|c|c|c|c|c|c|c|}
\hline & \multicolumn{4}{|c|}{$\begin{array}{c}\text { Japan } \\
\end{array}$} & \multicolumn{4}{|c|}{ Korea } & \multicolumn{4}{|c|}{ Latvia } \\
\hline & ROA(EBIT) & ROK(EBIT) & $\operatorname{ROA}(\mathbf{P} / \mathbf{L})$ & ROK & ROA(EBIT) & ROK(EBIT) & $\operatorname{ROA}(\mathbf{P} / \mathrm{L})$ & ROK & ROA(EBIT) & ROK(EBIT) & ROA(P/L) & ROK \\
\hline Listing (dummy) & $\begin{array}{l}-0.253^{*} \\
(-2.45)\end{array}$ & $\begin{array}{l}0.0235 \\
(0.61)\end{array}$ & $\begin{array}{c}-0.00428 \\
(-0.05)\end{array}$ & $\begin{array}{l}8.477^{*} \\
(2.19)\end{array}$ & $\begin{array}{l}-0.985^{*} \\
(-2.14)\end{array}$ & $\begin{array}{c}0.124 * * \\
(3.02)\end{array}$ & $\begin{array}{c}-1.968 * * * \\
(-9.41)\end{array}$ & $\begin{array}{l}9.940 \\
(1.86)\end{array}$ & $\begin{array}{l}-0.371 \\
(-0.11)\end{array}$ & $\begin{array}{l}-0.314 \\
(-0.43)\end{array}$ & $\begin{array}{r}-0.0240 \\
(-0.01)\end{array}$ & $\begin{array}{l}-74.17 \\
(-1.03)\end{array}$ \\
\hline L.Size & $\begin{array}{c}0.649 * * * \\
(38.76)\end{array}$ & $\begin{array}{c}0.00330 \\
(0.53)\end{array}$ & $\begin{array}{c}0.406^{* * * *} \\
(26.37)\end{array}$ & $\begin{array}{c}-1.956^{* *} \\
(-3.11)\end{array}$ & $\begin{array}{c}-1.268^{* * *} \\
(-23.93)\end{array}$ & $\begin{array}{c}-0.157 * * * \\
(-32.98)\end{array}$ & $\begin{array}{c}-1.393 * * * \\
(-57.83)\end{array}$ & $\begin{array}{c}-17.15^{* * *} \\
(-27.78)\end{array}$ & $\begin{array}{c}0.688^{* * * *} \\
(4.95)\end{array}$ & $\begin{array}{c}-0.0445 \\
(-1.35)\end{array}$ & $\begin{array}{c}0.355^{* * * *} \\
(3.57)\end{array}$ & $\begin{array}{l}-3.903 \\
(-1.21)\end{array}$ \\
\hline Age & $\begin{array}{c}-0.0468 * * * \\
(-35.13)\end{array}$ & $\begin{array}{c}-0.00728^{* * *} \\
(-14.76)\end{array}$ & $\begin{array}{c}-0.0496 * * * \\
(-40.50)\end{array}$ & $\begin{array}{c}-0.800^{* * * *} \\
(-16.04)\end{array}$ & $\begin{array}{c}-0.0208^{*} \\
(-2.37)\end{array}$ & $\begin{array}{c}-0.00585 * * * \\
(-7.43)\end{array}$ & $\begin{array}{c}-0.0256 * * * \\
(-6.40)\end{array}$ & $\begin{array}{c}-0.464 * * * \\
(-4.54)\end{array}$ & $\begin{array}{c}-0.161 * * * \\
(-4.62)\end{array}$ & $\begin{array}{c}-0.0273^{* * * *} \\
(-3.52)\end{array}$ & $\begin{array}{c}-0.191 * * * \\
(-7.65)\end{array}$ & $\begin{array}{c}-2.265^{* *} \\
(-2.98)\end{array}$ \\
\hline L.D/A (ratio) & $\begin{array}{c}0.0750 \\
(0.83)\end{array}$ & $\begin{array}{c}-0.0677^{*} \\
(-2.02)\end{array}$ & $\begin{array}{c}-2.241 * * * \\
(-26.90)\end{array}$ & $\begin{array}{c}-23.47^{* * * *} \\
(-6.91)\end{array}$ & $\begin{array}{c}-3.286^{* * *} \\
(-10.35)\end{array}$ & $\begin{array}{c}-0.450 * * * \\
(-15.83)\end{array}$ & $\begin{array}{c}-7.807 * * * \\
(-54.21)\end{array}$ & $\begin{array}{c}-57.69 * * * \\
(-15.65)\end{array}$ & $\begin{array}{l}1.692 \\
(1.83)\end{array}$ & $\begin{array}{c}-0.741^{* * * *} \\
(-3.47)\end{array}$ & $\begin{array}{l}-1.065 \\
(-1.61)\end{array}$ & $\begin{array}{c}-65.20^{* *} \\
(-3.11)\end{array}$ \\
\hline Constant & $\begin{array}{l}-0.640 \\
(-1.15)\end{array}$ & $\begin{array}{l}0.407^{*} \\
(1.97)\end{array}$ & $\begin{array}{c}3.294 * * * \\
(6.45)\end{array}$ & $\begin{array}{c}76.36 * * * \\
(3.66)\end{array}$ & $\begin{array}{l}6.931 \\
(0.32)\end{array}$ & $\begin{array}{l}1.027 \\
(0.53)\end{array}$ & $\begin{array}{l}10.80 \\
(1.10)\end{array}$ & $\begin{array}{l}118.1 \\
(0.47)\end{array}$ & $\begin{array}{l}1.841 \\
(0.40)\end{array}$ & $\begin{array}{l}1.338 \\
(1.36)\end{array}$ & $\begin{array}{l}4.951 \\
(1.51)\end{array}$ & $\begin{array}{l}112.4 \\
(1.17)\end{array}$ \\
\hline Observations & 132846 & 132674 & 132817 & 132645 & 109704 & 109609 & 109571 & 109480 & 15685 & 13910 & 15517 & 13814 \\
\hline Adjusted R-squared & 0.034 & 0.002 & 0.038 & 0.004 & 0.013 & 0.021 & 0.087 & 0.015 & 0.013 & 0.031 & 0.015 & 0.026 \\
\hline
\end{tabular}


Table A3.8: Benchmark Panel Regressions for MPK

The dependent variable is MPK, proxied by ROA or ROK. Listing is the binary variable, taking the vlaue of one if a firm is listed and zero otherise. L.Size is the logarithm of the lagged total assets. Age is the years since incorporation. L.D/A is the lagged debt to asset ratio. 2-digit industry fixed effects are included but not reported in the results. The robust standard errors are reported in the parenthesis corrected for clustering at the industry level: *denotes significance at $10 \% ; * *$ at $5 \%$; and $* * *$ at $1 \%$

\begin{tabular}{|c|c|c|c|c|c|c|c|c|c|c|c|c|}
\hline & \multicolumn{4}{|c|}{ Romania } & \multicolumn{4}{|c|}{ Malaysia } & \multicolumn{4}{|c|}{ Netherlands } \\
\hline & ROA(EBIT) & ROK(EBIT) & $\operatorname{ROA}(\mathbf{P} / \mathrm{L})$ & ROK & ROA(EBIT) & ROK(EBIT) & $\operatorname{ROA}(\mathbf{P} / \mathrm{L})$ & ROK & ROA(EBIT) & ROK(EBIT) & ROA(P/L) & ROK \\
\hline Listing (dummy) & $\begin{array}{c}-9.481^{* * * *} \\
(-9.86)\end{array}$ & $\begin{array}{l}-0.112 \\
(-0.42)\end{array}$ & $\begin{array}{c}-3.357 * * * \\
(-4.80)\end{array}$ & $\begin{array}{l}-12.52 \\
(-0.49)\end{array}$ & $\begin{array}{l}0.207 \\
(0.08)\end{array}$ & $\begin{array}{l}-0.0261 \\
(-0.03)\end{array}$ & $\begin{array}{l}-0.245 \\
(-0.09)\end{array}$ & $\begin{array}{l}-4.369 \\
(-0.05)\end{array}$ & $\begin{array}{c}-4.728^{*} \\
(-2.22)\end{array}$ & $\begin{array}{l}2.740 \\
(0.46)\end{array}$ & $\begin{array}{l}-2.579 \\
(-1.76)\end{array}$ & $\begin{array}{l}285.7 \\
(0.48)\end{array}$ \\
\hline L.Size & $\begin{array}{c}-1.050^{* * * *} \\
(-21.57)\end{array}$ & $\begin{array}{c}-0.151^{* * * *} \\
(-10.05)\end{array}$ & $\begin{array}{c}-1.366^{* * *} \\
(-38.55)\end{array}$ & $\begin{array}{c}-15.58^{* * * *} \\
(-10.93)\end{array}$ & $\begin{array}{l}0.702 \\
(1.91)\end{array}$ & $\begin{array}{l}-0.229 \\
(-1.72)\end{array}$ & $\begin{array}{l}0.748^{*} \\
(2.08)\end{array}$ & $\begin{array}{l}-23.85 \\
(-1.86)\end{array}$ & $\begin{array}{l}0.382 \\
(1.24)\end{array}$ & $\begin{array}{l}-1.031 \\
(-1.19)\end{array}$ & $\begin{array}{r}-0.0497 \\
(-0.24)\end{array}$ & $\begin{array}{l}-105.0 \\
(-1.22)\end{array}$ \\
\hline Age & $\begin{array}{c}-0.334 * * * \\
(-26.79)\end{array}$ & $\begin{array}{c}-0.0714 * * * \\
(-19.42)\end{array}$ & $\begin{array}{c}-0.336 * * * \\
(-37.08)\end{array}$ & $\begin{array}{c}-6.609 * * * \\
(-18.93)\end{array}$ & $\begin{array}{l}-0.0726 \\
(-1.41)\end{array}$ & $\begin{array}{c}-0.0431 * \\
(-2.30)\end{array}$ & $\begin{array}{c}-0.0600 \\
(-1.19)\end{array}$ & $\begin{array}{l}-4.345^{*} \\
(-2.42)\end{array}$ & $\begin{array}{c}-0.0353^{*} \\
(-2.25)\end{array}$ & $\begin{array}{l}0.0121 \\
(0.28)\end{array}$ & $\begin{array}{c}-0.0316 \text { ** } \\
(-2.93)\end{array}$ & $\begin{array}{l}1.268 \\
(0.29)\end{array}$ \\
\hline L.D/A (ratio) & $\begin{array}{c}-3.565 \text { *** } \\
(-13.57)\end{array}$ & $\begin{array}{c}-1.056^{* * * *} \\
(-12.71)\end{array}$ & $\begin{array}{c}-5.799^{* * *} \\
(-30.34)\end{array}$ & $\begin{array}{c}-112.1 \text { **** } \\
(-14.19)\end{array}$ & $\begin{array}{l}-0.389 \\
(-0.14)\end{array}$ & $\begin{array}{l}-1.667 \\
(-1.62)\end{array}$ & $\begin{array}{l}-5.454 \\
(-1.96)\end{array}$ & $\begin{array}{c}-196.7 * \\
(-1.99)\end{array}$ & $\begin{array}{l}-5.506^{*} \\
(-2.06)\end{array}$ & $\begin{array}{l}5.779 \\
(0.77)\end{array}$ & $\begin{array}{c}-6.333^{* * *} \\
(-3.42)\end{array}$ & $\begin{array}{l}567.1 \\
(0.76)\end{array}$ \\
\hline Constant & $\begin{array}{l}27.10 \\
(1.42) \\
\end{array}$ & $\begin{array}{l}7.428 \\
(0.98) \\
\end{array}$ & $\begin{array}{l}29.64 * \\
(2.14) \\
\end{array}$ & $\begin{array}{l}602.3 \\
(0.84) \\
\end{array}$ & $\begin{array}{c}-27.88^{* *} \\
(-2.60) \\
\end{array}$ & $\begin{array}{l}5.066 \\
(1.30) \\
\end{array}$ & $\begin{array}{l}-22.98^{*} \\
(-2.19) \\
\end{array}$ & $\begin{array}{l}545.5 \\
(1.46) \\
\end{array}$ & $\begin{array}{l}9.639^{*} \\
(1.97) \\
\end{array}$ & $\begin{array}{l}8.806 \\
(0.64) \\
\end{array}$ & $\begin{array}{c}13.35^{* * *} \\
(3.98) \\
\end{array}$ & $\begin{array}{l}893.7 \\
(0.65) \\
\end{array}$ \\
\hline Observations & 123180 & 112851 & 122604 & 112493 & 357 & 357 & 356 & 356 & 1484 & 1447 & 1469 & 1432 \\
\hline Adjusted R-squared & 0.033 & 0.011 & 0.072 & 0.012 & 0.514 & -0.135 & 0.478 & -0.062 & -0.014 & -0.052 & 0.011 & -0.053 \\
\hline
\end{tabular}


Table A3.9: Benchmark Panel Regressions for MPK

The dependent variable is MPK, proxied by ROA or ROK. Listing is the binary variable, taking the vlaue of one if a firm is listed and zero otherise. L.Size is the logarithm of the lagged total assets. Age is the years since incorporation. L.D/A is the lagged debt to asset ratio. 2-digit industry fixed effects are included but not reported in the results. The robust standard errors are reported in the parenthesis corrected for clustering at the industry level: *denotes significance at $10 \%$; ** at $5 \%$; and $* * *$ at $1 \%$

\begin{tabular}{|c|c|c|c|c|c|c|c|c|c|c|c|c|}
\hline & \multicolumn{4}{|c|}{ Norway } & \multicolumn{4}{|c|}{ Poland } & \multicolumn{4}{|c|}{ Spain } \\
\hline & ROA(EBIT) & ROK(EBIT) & $\operatorname{ROA}(\mathbf{P} / \mathbf{L})$ & ROK & ROA(EBIT) & ROK(EBIT) & $\operatorname{ROA}(\mathbf{P} / \mathbf{L})$ & ROK & ROA(EBIT) & ROK(EBIT) & $\operatorname{ROA}(\mathbf{P} / \mathbf{L})$ & ROK \\
\hline Listing (dummy) & $\begin{array}{c}-22.38 * * * \\
(-5.39)\end{array}$ & $\begin{array}{c}-160.1^{* * *} \\
(-7.27)\end{array}$ & $\begin{array}{c}-17.05^{* * *} \\
(-4.62)\end{array}$ & $\begin{array}{c}-4704.3^{* * *} \\
(-3.24)\end{array}$ & $\begin{array}{l}-11.32^{*} \\
(-2.49)\end{array}$ & $\begin{array}{l}0.196 \\
(0.33)\end{array}$ & $\begin{array}{c}-6.417 * * * \\
(-7.18)\end{array}$ & $\begin{array}{l}84.92 \\
(1.42)\end{array}$ & $\begin{array}{c}-1.540^{*} \\
(-2.29)\end{array}$ & $\begin{array}{l}0.207 \\
(0.65)\end{array}$ & $\begin{array}{l}-1.005 \\
(-1.86)\end{array}$ & $\begin{array}{l}11.44 \\
(0.41)\end{array}$ \\
\hline L.Size & $\begin{array}{l}0.639^{*} \\
(2.20)\end{array}$ & $\begin{array}{l}5.088^{* *} \\
(3.08)\end{array}$ & $\begin{array}{l}0.536^{*} \\
(2.10)\end{array}$ & $\begin{array}{c}304.1^{* *} \\
(2.82)\end{array}$ & $\begin{array}{c}2.420^{* * *} \\
(5.41)\end{array}$ & $\begin{array}{c}-0.383 * * * \\
(-6.20)\end{array}$ & $\begin{array}{l}0.124 \\
(1.41)\end{array}$ & $\begin{array}{c}-41.88^{* * * *} \\
(-6.71)\end{array}$ & $\begin{array}{c}0.974^{* * *} \\
(54.44)\end{array}$ & $\begin{array}{c}0.0178^{*} \\
(2.08)\end{array}$ & $\begin{array}{l}0.867 * * * \\
(60.11)\end{array}$ & $\begin{array}{c}3.903^{* * * *} \\
(5.17)\end{array}$ \\
\hline Age & $\begin{array}{c}0.111^{* * *} \\
(4.55)\end{array}$ & $\begin{array}{l}0.183 \\
(1.41)\end{array}$ & $\begin{array}{l}0.0292 \\
(1.33)\end{array}$ & $\begin{array}{l}4.316 \\
(0.50)\end{array}$ & $\begin{array}{c}-0.0910^{* *} \\
(-2.67)\end{array}$ & $\begin{array}{c}-0.0114^{* *} \\
(-2.58)\end{array}$ & $\begin{array}{c}-0.0706^{* * *} \\
(-10.56)\end{array}$ & $\begin{array}{c}-1.044^{*} \\
(-2.34)\end{array}$ & $\begin{array}{c}-0.121^{* * *} \\
(-50.07)\end{array}$ & $\begin{array}{c}-0.0208 * * * \\
(-18.24)\end{array}$ & $\begin{array}{c}-0.122 * * * \\
(-62.76)\end{array}$ & $\begin{array}{c}-2.121 * * * \\
(-21.11)\end{array}$ \\
\hline L.D/A (ratio) & $\begin{array}{c}12.63^{* * *} \\
(7.27)\end{array}$ & $\begin{array}{l}8.285 \\
(0.86)\end{array}$ & $\begin{array}{c}6.933^{* * * *} \\
(4.54)\end{array}$ & $\begin{array}{l}-11.42 \\
(-0.02)\end{array}$ & $\begin{array}{l}0.929 \\
(0.61)\end{array}$ & $\begin{array}{l}0.296 \\
(1.50)\end{array}$ & $\begin{array}{c}-1.892^{* * *} \\
(-6.39)\end{array}$ & $\begin{array}{l}11.20 \\
(0.56)\end{array}$ & $\begin{array}{c}2.716 * * * \\
(29.49)\end{array}$ & $\begin{array}{c}-0.163^{* * *} \\
(-3.72)\end{array}$ & $\begin{array}{c}-0.418^{* * *} \\
(-5.63)\end{array}$ & $\begin{array}{c}-43.88^{* * * *} \\
(-11.36)\end{array}$ \\
\hline Constant & $\begin{array}{l}9.096 \\
(0.41) \\
\end{array}$ & $\begin{array}{l}87.48 \\
(0.75) \\
\end{array}$ & $\begin{array}{l}14.01 \\
(0.72) \\
\end{array}$ & $\begin{array}{l}759.9 \\
(0.10) \\
\end{array}$ & $\begin{array}{l}16.77 \\
(0.37) \\
\end{array}$ & $\begin{array}{l}3.837 \\
(0.66) \\
\end{array}$ & $\begin{array}{c}33.60^{* * *} \\
(3.82) \\
\end{array}$ & $\begin{array}{l}417.5 \\
(0.71) \\
\end{array}$ & $\begin{array}{l}6.838 \\
(1.29) \\
\end{array}$ & $\begin{array}{l}0.377 \\
(0.15) \\
\end{array}$ & $\begin{array}{l}7.795 \\
(1.83) \\
\end{array}$ & $\begin{array}{l}32.91 \\
(0.15) \\
\end{array}$ \\
\hline Observations & 2980 & 2756 & 2969 & 2746 & 14116 & 13747 & 14059 & 13705 & 317753 & 312137 & 317387 & 311822 \\
\hline Adjusted R-squared & 0.112 & 0.071 & 0.053 & 0.099 & -0.004 & 0.011 & 0.024 & 0.012 & 0.043 & 0.003 & 0.058 & 0.004 \\
\hline
\end{tabular}


Table A3.10: Benchmark Panel Regressions for MPK

The dependent variable is MPK, proxied by ROA or ROK. Listing is the binary variable, taking the vlaue of one if a firm is listed and zero otherise. L.Size is the logarithm of the lagged total assets. Age is the years since incorporation. L.D/A is the lagged debt to asset ratio. 2-digit industry fixed effects are included but not reported in the results. The robust standard errors

\begin{tabular}{|c|c|c|c|c|c|c|c|c|c|c|c|c|}
\hline & \multicolumn{4}{|c|}{ Sweden } & \multicolumn{4}{|c|}{ Taiwan } & \multicolumn{4}{|c|}{$\overline{\text { US }}$} \\
\hline & ROA(EBIT) & ROK(EBIT) & $\operatorname{ROA}(\mathbf{P} / \mathrm{L})$ & ROK & ROA(EBIT) & ROK(EBIT, & ROA(P/L) & ROK F & ROA(EBIT) & ROK(EBIT) & ROA(P/L) & ROK \\
\hline Listing (dummy) & $\begin{array}{c}-21.620 * * * \\
(-39.260)\end{array}$ & $\begin{array}{l}-5.929^{* * *} \\
(-10.000)\end{array}$ & $\begin{array}{c}-16.920 * * * \\
(-33.340)\end{array}$ & $\begin{array}{c}-338.500 \text { *** } \\
(-8.200)\end{array}$ & $\begin{array}{c}0.413 \\
(0.620)\end{array}$ & $\begin{array}{c}0.092 \\
(1.050)\end{array}$ & $\begin{array}{c}0.111 \\
(0.160)\end{array}$ & $\begin{array}{c}8.388 \\
(1.200)\end{array}$ & $\begin{array}{l}-61.280 \\
(-0.170)\end{array}$ & $\begin{array}{l}-30.740 \\
(-0.840)\end{array}$ & $\begin{array}{c}-13.600^{* * * *} \\
(-3.560)\end{array}$ & $\begin{array}{c}-1509.800 \\
(-0.45)\end{array}$ \\
\hline L.Size & $\begin{array}{l}0.177^{*} \\
(2.380)\end{array}$ & $\begin{array}{c}-0.144 \\
(-1.800)\end{array}$ & $\begin{array}{c}-0.054 \\
(-0.800)\end{array}$ & $\begin{array}{c}-26.750 * * * \\
(-4.840)\end{array}$ & $\begin{array}{l}1.409 * * * \\
(13.320)\end{array}$ & $\begin{array}{c}0.056^{* * * *} \\
(4.050)\end{array}$ & $\begin{array}{l}1.433 * * * \\
(12.590)\end{array}$ & $\begin{array}{c}5.104 * * * \\
(4.570)\end{array}$ & $\begin{array}{c}53.090 * * * \\
(4.850)\end{array}$ & $\begin{array}{c}5.247 * * * \\
(4.660)\end{array}$ & $\begin{array}{l}3.676^{* * *} \\
(30.150)\end{array}$ & $\begin{array}{c}310.500^{* * *} \\
(2.890)\end{array}$ \\
\hline Age & $\begin{array}{l}-0.012^{*} \\
(-2.190)\end{array}$ & $\begin{array}{c}-0.006 \\
(-1.100)\end{array}$ & $\begin{array}{c}-0.021^{* * *} \\
(-4.230)\end{array}$ & $\begin{array}{c}-1.053^{* *} \\
(-2.670)\end{array}$ & $\begin{array}{c}0.020 \\
(1.500)\end{array}$ & $\begin{array}{c}-0.002 \\
(-1.020)\end{array}$ & $\begin{array}{l}0.035^{*} \\
(2.510)\end{array}$ & $\begin{array}{c}-0.108 \\
(-0.790)\end{array}$ & $\begin{array}{c}-0.846 \\
(-1.080)\end{array}$ & $\begin{array}{c}0.014 \\
(0.170)\end{array}$ & $\begin{array}{c}0.054^{* * *} \\
(6.390)\end{array}$ & $\begin{array}{l}1.659 \\
(0.220)\end{array}$ \\
\hline L.D/A (ratio) & $\begin{array}{c}-3.575 * * * \\
(-9.400)\end{array}$ & $\begin{array}{l}-1.038^{*} \\
(-2.530)\end{array}$ & $\begin{array}{c}-8.703 * * * \\
(-25.150)\end{array}$ & $\begin{array}{c}-212.700^{* * * *} \\
(-7.540)\end{array}$ & $\begin{array}{c}-7.693 * * * \\
(-9.560)\end{array}$ & $\begin{array}{c}-0.367 * * * \\
(-3.480)\end{array}$ & $\begin{array}{c}-10.410^{* * *} \\
(-12.030)\end{array}$ & $\begin{array}{c}-40.580^{* * * *} \\
(-4.790)\end{array}$ & $\begin{array}{l}-80.750 \\
(-0.780)\end{array}$ & $\begin{array}{c}-1.433 \\
(-0.140)\end{array}$ & $\begin{array}{c}-8.164 * * * \\
(-7.240)\end{array}$ & $\begin{array}{l}-40.260 \\
(-0.040)\end{array}$ \\
\hline Constant & $\begin{array}{l}10.850 \\
(1.160) \\
\end{array}$ & $\begin{array}{c}7.466 \\
(0.750) \\
\end{array}$ & $\begin{array}{c}8.967 \\
(1.050) \\
\end{array}$ & $\begin{array}{r}634.900 \\
(0.930) \\
\end{array}$ & $\begin{array}{c}-7.574 * * \\
(-3.260) \\
\end{array}$ & $\begin{array}{c}-0.379 \\
(-1.240) \\
\end{array}$ & $\begin{array}{c}-7.795 * * \\
(-3.120) \\
\end{array}$ & $\begin{array}{l}-33.420 \\
(-1.360) \\
\end{array}$ & $\begin{array}{c}-539.100 \\
(-0.750) \\
\end{array}$ & $\begin{array}{l}-40.030 \\
(-0.490) \\
\end{array}$ & $\begin{array}{c}-51.650^{* * * *} \\
(-5.560) \\
\end{array}$ & $\begin{array}{c}-3175.400 \\
(-0.390) \\
\end{array}$ \\
\hline Observations & 35217 & 34451 & 35136 & 34373 & 5298 & 5298 & 5295 & 5295 & 7607 & 7599 & 7449 & 7448 \\
\hline Adjusted R-squared & 0.079 & 0.010 & 0.082 & 0.013 & 0.076 & -0.010 & 0.071 & -0.000 & 0.080 & -0.013 & 0.234 & -0.018 \\
\hline
\end{tabular}


Table A3.11: Benchmark Panel Regressions for MPK

The dependent variable is MPK, proxied by ROA or ROK. Listing is the binary variable, taking the vlaue of one if a firm is listed and zero otherise. L.Size is the logarithm of the lagged total assets. Age is the years since incorporation. L D/A is the lagged debt to asset ratio. 2-digit industry fixed effects are included but not reported in the results. The robust standard errors are reported in the parenthesis corrected for clustering at the industry level: *denotes significance at $10 \%$;** at $5 \%$; and *** at $1 \%$

\begin{tabular}{|c|c|c|c|c|c|c|c|c|c|c|c|c|}
\hline & \multicolumn{4}{|c|}{ Ukraine } & \multicolumn{4}{|c|}{ UK } & \multicolumn{4}{|c|}{ Vietnam } \\
\hline & ROA(EBIT) & ROK(EBIT) & $\operatorname{ROA}(\mathbf{P} / \mathrm{L})$ & ROK & ROA(EBIT) & ROK(EBIT) & $\operatorname{ROA}(\mathrm{P} / \mathrm{L})$ & ROK & ROA(EBIT) & ROK(EBIT) & $\operatorname{ROA}(\mathrm{P} / \mathrm{L})$ & ROK \\
\hline \multirow{2}{*}{ Listing (dummy) } & -1.776 & -0.730 & $-2.430^{* *}$ & -60.270 & $-17.180^{* * *}$ & $-7.131 * * *$ & $-11.480^{* * *}$ & $-390.700^{* * *}$ & -0.009 & -0.299 & $1.462 * *$ & -16.130 \\
\hline & $(-0.670)$ & $(-1.700)$ & $(-3.240)$ & $(-1.770)$ & $(-29.470)$ & $(-7.430)$ & $(-31.110)$ & $(-4.310)$ & $(-0.000)$ & $(-0.840)$ & $(3.280)$ & $(-0.460)$ \\
\hline \multirow{2}{*}{ L.Size } & $-0.395^{* * *}$ & $0.089^{* * *}$ & $-0.264 * * *$ & $5.462^{* *}$ & $1.231^{* * *}$ & $0.615^{* *}$ & $0.697 * * *$ & 12.520 & $1.657 * * *$ & $0.097^{* *}$ & $0.908^{* * *}$ & $8.208^{*}$ \\
\hline & $(-3.400)$ & $(4.290)$ & $(-7.990)$ & $(3.280)$ & (10.870) & $(3.200)$ & $(9.710)$ & $(0.690)$ & $(4.300)$ & $(2.760)$ & $(23.340)$ & $(2.370)$ \\
\hline \multirow{2}{*}{ Age } & $-0.050^{*}$ & $-0.030 * * *$ & $-0.061 * * *$ & $-2.710^{* * *}$ & $0.043 * * *$ & 0.003 & $0.020 * * *$ & -0.952 & 0.059 & -0.006 & $0.090 * * *$ & -0.226 \\
\hline & $(-2.250)$ & $(-8.260)$ & $(-9.760)$ & $(-9.450)$ & $(6.810)$ & $(0.250)$ & $(4.950)$ & $(-0.990)$ & $(0.580)$ & $(-0.700)$ & (8.770) & $(-0.270)$ \\
\hline \multirow{2}{*}{ L.D/A (ratio) } & $8.254 * * *$ & $1.270^{* * *}$ & $4.292 * * *$ & $95.640 * * *$ & $9.990 * * *$ & $3.484 * *$ & $2.040^{* * *}$ & 107.500 & -1.173 & 0.225 & $-0.656 * * *$ & 4.161 \\
\hline & (10.790) & $(9.640)$ & (19.830) & $(9.110)$ & (13.680) & $(2.900)$ & $(4.480)$ & $(0.960)$ & $(-0.630)$ & $(1.320)$ & $(-3.490)$ & $(0.250)$ \\
\hline \multirow{2}{*}{ Constant } & -29.740 & 0.768 & 2.082 & 152.700 & $-15.330^{* * *}$ & -1.639 & -4.845 & 169.800 & -11.460 & -0.696 & $-7.388^{* * *}$ & -55.250 \\
\hline & $(-1.620)$ & $(0.250)$ & $(0.390)$ & $(0.610)$ & $(-3.500)$ & $(-0.230)$ & $(-1.720)$ & $(0.250)$ & $(-0.720)$ & $(-0.510)$ & $(-4.580)$ & $(-0.420)$ \\
\hline Observations & 110105 & 96494 & 108365 & 95396 & 18602 & 18439 & 18461 & 18319 & 28038 & 21481 & 27960 & 21452 \\
\hline Adjusted R-squared & 0.004 & 0.007 & 0.037 & 0.007 & 0.090 & 0.008 & 0.084 & 0.005 & -0.003 & -0.003 & 0.079 & -0.004 \\
\hline
\end{tabular}

